\title{
Modification and re-validation of the ethyl acetate-based multi-residue method for pesticides in produce
}

\author{
Hans G. J. Mol • Astrid Rooseboom • Ruud van Dam • \\ Marleen Roding $\cdot$ Karin Arondeus $\cdot$ Suryati Sunarto
}

Received: 23 March 2007 / Revised: 4 May 2007 / Accepted: 8 May 2007 / Published online: 12 June 2007

(C) Springer-Verlag 2007

\begin{abstract}
The ethyl acetate-based multi-residue method for determination of pesticide residues in produce has been modified for gas chromatographic (GC) analysis by implementation of dispersive solid-phase extraction (using primary-secondary amine and graphitized carbon black) and large-volume $(20 \mu \mathrm{L})$ injection. The same extract, before clean-up and after a change of solvent, was also analyzed by liquid chromatography with tandem mass spectrometry (LC-MS-MS). All aspects related to sample preparation were re-assessed with regard to ease and speed of the analysis. The principle of the extraction procedure (solvent, salt) was not changed, to avoid the possibility invalidating data acquired over past decades. The modifications were made with techniques currently commonly applied in routine laboratories, GC-MS and LC-MS-MS, in mind. The modified method enables processing (from homogenization until final extracts for both GC and LC) of 30 samples per eight hours per person. Limits of quantification (LOQs) of $0.01 \mathrm{mg} \mathrm{kg}^{-1}$ were achieved with both GC-MS (full-scan acquisition, $10 \mathrm{mg}$ matrix equivalent injected) and LC-MSMS ( $2 \mathrm{mg}$ injected) for most of the pesticides. Validation data for 341 pesticides and degradation products are presented. A compilation of analytical quality-control data for pesticides routinely analyzed by GC-MS (135 compounds) and LC-
\end{abstract}

H. G. J. Mol ( $\bowtie)$

Rikilt Institute of Food Safety, Pesticides and Contaminants, P.O. Box 230, 6700 AE Wageningen, The Netherlands e-mail: hans.mol@wur.nl

\section{A. Rooseboom}

TNO-Blgg-AgriQ,

P.O. Box 392, 6700 AJ Wageningen, The Netherlands

R. van Dam $\cdot$ M. Roding $\cdot$ K. Arondeus $\cdot$ S. Sunarto Department Analytical Research, TNO Quality of Life, P.O. Box 513, 3700 AJ Zeist, The Netherlands
MS-MS (136 compounds) in over 100 different matrices, obtained over a period of 15 months, are also presented and discussed. At the $0.05 \mathrm{mg} \mathrm{kg}^{-1}$ level acceptable recoveries were obtained for 93\% (GC-MS) and 92\% (LC-MS-MS) of pesticide-matrix combinations.

Keywords Foods/Beverages · Pesticides · GC-MS · LC-MS/MS · Multi-residue analysis

\section{Introduction}

For monitoring and control of pesticide residues, multiresidue methods are very cost-effective and are used in many laboratories. The pesticides are usually first extracted with an organic solvent of high or medium polarity. Typical solvents used for this purpose are acetone [1-4], ethyl acetate [5-26] (Table 1), and acetonitrile [26-31]. With all three options, pesticides are partitioned between an aqueous phase and an organic phase. With acetone and acetonitrile this is done in two successive steps, with ethyl acetate in one step. With regard to extraction efficiency, ethyl acetate has been shown to be equivalent to the water-miscible solvents for both polar and non-polar pesticides in vegetables, fruit, and dry products (after addition of water) [6, 7, 26, 32]. It is also suitable for products with a high fat content-because of the solubility of fat in ethyl acetate, pesticides are released and extracted efficiently. The extract obtained is compatible with gel-permeation chromatography (GPC), the clean-up procedure most suitable for this type of sample. Ethyl acetate is very suitable for GC analysis. It has good wettability in GC (pre)columns; this is of benefit for solvent trapping of the most volatile analytes, which is required for refocusing after injection. Its vapor pressure and expansion volume during evaporation also favor large- 


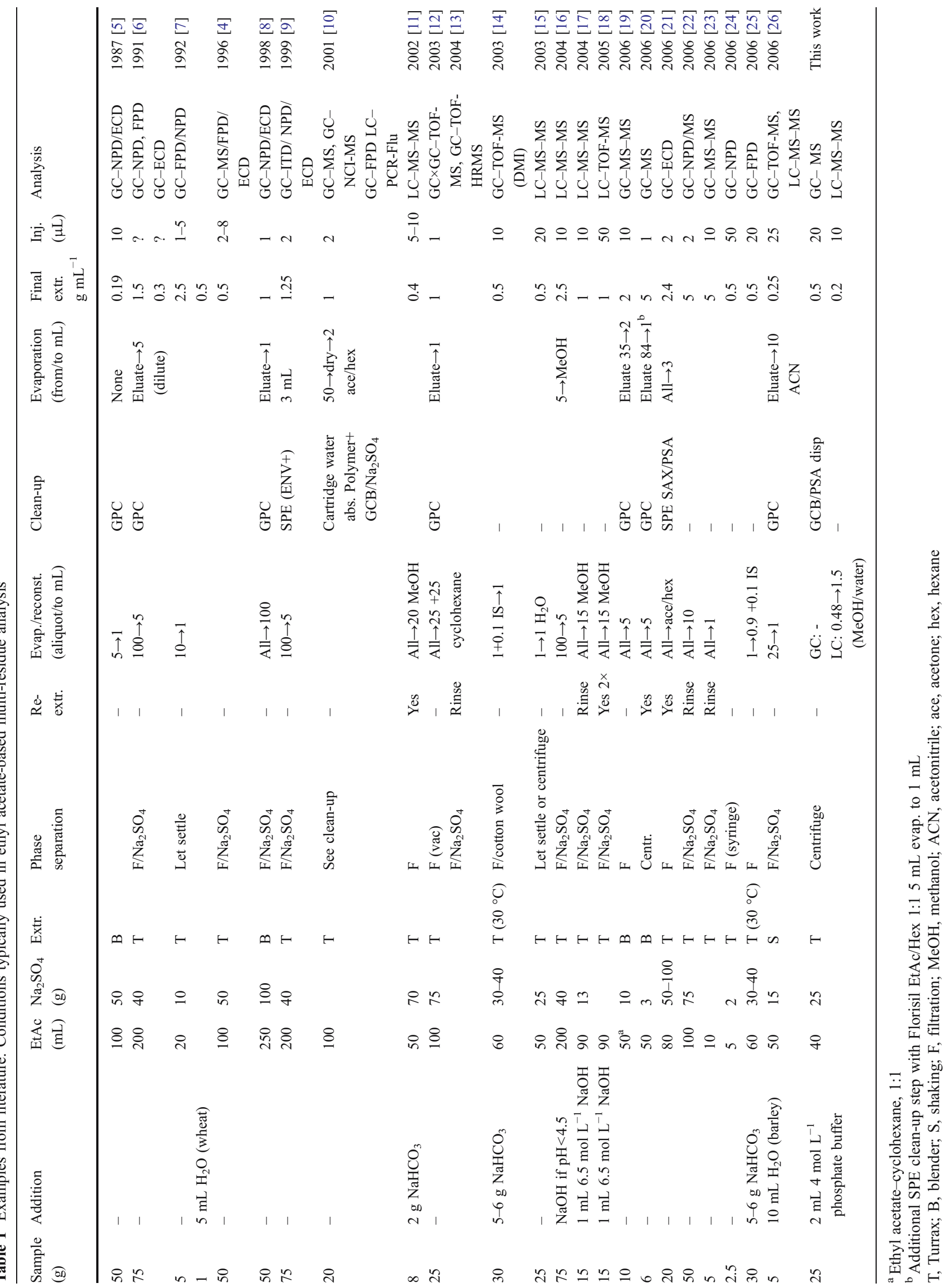


volume injection. Finally, it is compatible with all GC detectors. The same extract can also be used for LC analysis, after a solvent change into, e.g., methanol [11, 15$18,26]$, as is done for acetone-based methods also [33].

Although multi-residue methods based on ethyl acetate extraction have been used for more than 20 years, and continue to be used in many laboratories (they are, for example, the official methods in Sweden and Spain and are also commonly used in the Netherlands, UK, Czech Republic, Japan, and China), the methods described in the literature frequently include steps that make them, in our opinion, unnecessary laborious. Such steps include repeated extraction, filtration, clean-up steps involving GPC for nonfatty matrices, column chromatography or solid phase extraction (SPE) manifolds and evaporative concentration. Typical examples are given in Table 1. It will be shown in this paper that most of the laborious steps can be replaced by more efficient alternatives - repeated extraction is not required, an aliquot is taken after settling or centrifugation rather than filtration, use of GCB instead of GPC for removal of chlorophyll, use of dispersive SPE instead of classical SPE for clean-up (analogous to an acetonitrile-based method [29]), and injection of larger volumes into the GC instead of manual evaporative concentration.

The objective of the work discussed in this paper was to update and improve the ethyl acetate-based multi-residue method for pesticides in vegetables and fruit in respect of straightforwardness, robustness, and ease and speed of sample and extract handling. Aspects studied include dispersive clean-up using combined GCB/PSA, the possibility of preventing unacceptable adsorption of "planar" pesticides by GCB, by addition of toluene, and large-volume $(20 \mu \mathrm{L})$ injection in GC. The method has been validated for 341 pesticides and degradation products which are analyzed by GC-MS or LC-MS-MS. For the latter the initial raw extract was used and injected after a solvent change to methanol-water. The suitability of the method as a multiresidue, multi-matrix method is evaluated by use of analytical quality-control data generated during 15 months for 271 pesticides and degradation products for over 100 different matrices, including less common and exotic crops. Results obtained for proficiency test samples during three years are also presented.

\section{Experimental}

Chemicals and reagents

Pesticide reference standards were obtained from C.N. Schmidt (Amsterdam, The Netherlands). For GC-MS a mixed stock solution containing 135 pesticides (Table 7; concentration $50 \mathrm{mg} \mathrm{L}^{-1}$ for each pesticide) was obtained from Alltech-Grace (Breda, The Netherlands). The full chemical names of the metabolites of phenmedipham and pyridate are methyl $N$-(3-hydroxyphenyl)carbamate and 3-phenyl-4-hydroxy-6-chloropyridazine, respectively. Solvents were from J.T. Baker (ethyl acetate, Resi-analysed; Deventer, The Netherlands), Labscan (toluene, Pestiscan), and Rathburn (methanol). Anhydrous sodium sulfate, ammonium formate, potassium dihydrogen phosphate, disodium hydrogen phosphate, acetic acid, and diethylene glycol (all p.A. quality) were from Merck. Water was purified by use of a MilliQ reagent-water system (Millipore).

Bondesil primary secondary amine (PSA, $40 \mu \mathrm{m}$ ) was obtained from Varian (Middelburg, The Netherlands) and GCB (graphitized carbon black) was purchased as Supelclean ENVI-carb (120-400 mesh, Supelco, Zwijndrecht, The Netherlands).

For GC-MS, in addition to the mixed stock solution, individual stock solutions of other pesticides were prepared in ethyl acetate. From these, additional mixed solutions were prepared in ethyl acetate. For LC-MS-MS analysis, individual stock solutions were prepared in methanol. Mixed solutions were prepared from the individual stock solutions and diluted with methanol. The mixed solutions were used for fortification of samples and for preparation of matrix-matched standards.

The extraction solvent was a solution of internal standard ( $0.05 \mathrm{mg} \mathrm{L}^{-1}$ antor (diethatyl-ethyl)) in ethyl acetate. Matrixmatched standards were prepared by addition of mixed solutions to control sample extracts. Dilution of the sample extract with mixed solution was never more than $10 \%$.

\section{Instrumentation}

\section{GC-MS analysis}

GC-MS analysis was performed with a model 8000 Top GC equipped with a Best PTV (programmed temperature vaporizer) injector, an AS800 autosampler, and a Voyager mass spectrometer (Interscience, Breda, The Netherlands). The instrument was controlled by Masslab software. The injector was equipped with a $1 \mathrm{~mm}$ i.d. liner with porous sintered glass on the inner surface. The GC was equipped with a $30 \mathrm{~m} \times 0.25 \mathrm{~mm}$ i.d., $0.25 \mu \mathrm{m}$ film, HP-5-MS column and a $2.5 \mathrm{~m}$ precolumn (same as the analytical column, connected by means of a press-fit connector).

For PTV injection in solvent-vent mode $20 \mu \mathrm{L}$ was injected at $5 \mu \mathrm{L} \mathrm{s}^{-1}$. The solvent was vented at $50^{\circ} \mathrm{C}$ in

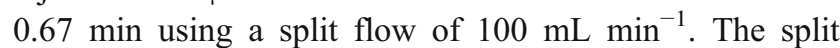
valve was then closed and the analytes retained in the liner were transferred to the $\mathrm{GC}$ column by ramping the temperature at $10^{\circ} \mathrm{s}^{-1}$ to $300^{\circ} \mathrm{C}$. Total transfer time was $2.5 \mathrm{~min}$ after which the split was re-opened. 
Helium was used as carrier gas at constant flow $(1.5 \mathrm{~mL}$ $\min ^{-1}$ ). The oven temperature was maintained at $90^{\circ} \mathrm{C}$ for 2 min after injection then programmed at $10^{\circ} \mathrm{min}^{-1}$ to $300^{\circ} \mathrm{C}$ which was maintained for $10 \mathrm{~min}$. The transfer line to the MS was maintained at $305^{\circ} \mathrm{C}$.

Mass spectrometry was performed with electron-impact (EI) ionization (electron energy $70 \mathrm{eV}$ ) at a source temperature of $200^{\circ} \mathrm{C}$. Data were acquired in full-scan mode $(\mathrm{m} / \mathrm{z} 60-400)$, after a solvent delay of $5.5 \mathrm{~min}$, until $30 \mathrm{~min}$. Scan time and inter-scan delay were 0.3 and $0.1 \mathrm{~s}$, respectively, resulting in 2.5 scans s$^{-1}$. The detector potential was $450 \mathrm{~V}$.

Masslab software (Interscience, The Netherlands) and an Excel macro developed in-house were used for data handling and quantitative data evaluation.

\section{LC-MS-MS analysis}

LC was performed with an Agilent, model 1100 instrument comprising degas-unit, pump, autosampler, and column oven. A $4 \mathrm{~mm} \times 2 \mathrm{~mm}$ i.d. $\mathrm{C}_{18}$ guard column (Phenomenex) and a $150 \mathrm{~mm} \times 3 \mathrm{~mm}$ i.d. LC column (Aqua, $5 \mu \mathrm{m} \mathrm{C}_{18}$, Phenomenex) were coupled to a triple-quadrupole mass spectrometer (model API2000 or API3000, Applied Biosystems, Nieuwerkerk a/d Yssel, The Netherlands). Analyst 1.2 and, later, 1.4 were used for instrument control and data handling. Additional data processing was performed using an Excel macro developed in-house.

Compounds were separated by elution with a gradient prepared from methanol-water- $1 \mathrm{~mol} \mathrm{~L}^{-1}$ ammonium formate solution, 20:79.5:0.5 (component A) and methanolwater-1 mol L ${ }^{-1}$ ammonium formate solution, 90:9.5:0.5 (component B). The composition was changed from $100 \%$ A to $100 \% \mathrm{~B}$ in $8 \mathrm{~min}$ and was then isocratic until $24 \mathrm{~min}$. The composition was then changed back to $100 \% \mathrm{~A}$ in $1 \mathrm{~min}$ and the column was re-equilibrated for $10 \mathrm{~min}$ before the next injection. The flow rate was $0.3 \mathrm{~mL} \mathrm{~min}^{-1}$ which was introduced into the MS without splitting. The injection volume was $20 \mu \mathrm{L}$ and $10 \mu \mathrm{L}$ for the API2000 and API3000, respectively.

Data were acquired in multiple-reaction-monitoring (MRM) mode. Electrospray ionization (ESI) (called turbo ion spray for the instruments used) mass spectrometry was performed in positive-ion mode. For the API2000 the nebulizer gas, turbo gas, and curtain gas were 20, 50, and 40 arbitrary units (a.u.), respectively. The ion-spray potential was $5000 \mathrm{~V}$. Nitrogen was used as collision gas (4 psi). For the API3000 the nebulizer gas and curtain gas were 12 and 10 a.u. and the turbo gas was $7.5 \mathrm{~L} \mathrm{~min}^{-1}$. The ion spray potential was $2000 \mathrm{~V}$. Nitrogen was used as collision gas (4 psi). For both instruments, the pause time was $5 \mathrm{~ms}$. The dwell times for the pesticide transitions varied between 10 and $25 \mathrm{~ms}$. The precursor and product ions and the collision energy (data for API3000) for each pesticide or degradation product are listed in Table 8 . In the acquisition method one transition for each pesticide was measured. All transitions were acquired in one time window. The total cycle time was $2.24 \mathrm{~s}$ resulting in $8-10$ data points across the peak. To measure the second transition a second method was created and run if confirmation was needed.

\section{Sample preparation}

Vegetable and fruit samples were taken from batches of samples as received from the food industry and trade for routine multi-residue analysis. After removal of stalks, caps, stems, etc., as prescribed by 90/642/EEC Annex I [34], an amount corresponding, at least, to the minimum size of laboratory samples (usually $1-2 \mathrm{~kg}$ [35]) was homogenized in a large-scale Stephan food cutter. A subsample $(25 \mathrm{~g})$ was weighed into a centrifuge tube. Fortification was performed

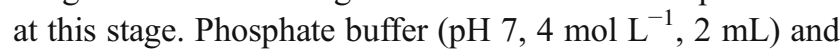
extraction solution (ethyl acetate with internal standard, $40 \mathrm{~mL}$ ) were then added. Just before Turrax extraction anhydrous sodium sulfate $(25 \mathrm{~g})$ was added. After Turrax extraction $(1 \mathrm{~min})$ the tubes were centrifuged (sets of four).

For GC-MS analysis, Eppendorf cups were prefilled with $25 \mathrm{mg}$ PSA and $25 \mathrm{mg}$ GCB. To avoid a weighing step, scoops were made in-house for this purpose. Their accuracy was established to be $25 \pm 2 \mathrm{mg}(n=10)$. For cleanup, $0.8 \mathrm{~mL}$ extract and $0.2 \mathrm{~mL}$ toluene were added to the cup with the SPE materials. The cups were then closed and the samples were vortex mixed for $30 \mathrm{~s}$ and centrifuged (up to 24 at one time). One aliquot was transferred to an autosampler vial with insert, and a second aliquot was transferred to an autosampler vial and stored under refrigeration as backup extract. The calculated amount of initial sample in the final extract was $0.5 \mathrm{~g} \mathrm{~mL}^{-1}$.

For LC-MS-MS analysis the initial extract $(3.2 \mathrm{~mL}$ for the API2000 and $0.48 \mathrm{~mL}$ for the API3000) was transferred to a disposable glass tube. After addition of a solution of diethylene glycol in methanol $(10 \%, 200 \mu \mathrm{L})$ the extract was evaporated to "dryness" under a gentle flow of nitrogen gas at $35^{\circ} \mathrm{C}$ (up to 36 tubes in a heater block). The residue was reconstituted in methanol $(1 \mathrm{~mL}$ and $0.75 \mathrm{~mL}$ for the API2000 and API3000, respectively), by use of vortex mixing and ultrasonication $(5 \mathrm{~min})$. The extract was then diluted 1:1 with component A. After centrifugation one aliquot was transferred to an autosampler vial with insert, and a second aliquot was transferred into an autosampler vial and stored under refrigeration as back-up extract. The final extract concentration was $1 \mathrm{~g} \mathrm{~mL}^{-1}$ and $0.2 \mathrm{~g} \mathrm{~mL}^{-1}$ for the API2000 and API3000, respectively.

For dry products (e.g. cereals) $5 \mathrm{~g}$ was weighed and $20 \mathrm{~mL}$ water was added. After soaking for $2 \mathrm{~h}$ samples were processed as described above. A larger amount of 
extract was taken for evaporation to compensate for the reduced amount of sample processed and to bring the final extract concentration to $0.2 \mathrm{~g} \mathrm{~mL}^{-1}$.

With the final method, one person can process 30 samples in eight hours. Here processing includes specific preparation before homogenization (i.e. removal of caps from strawberries, etc.), homogenization of the samples, extraction, cleaning the Turrax between samples, clean-up for GCMS, and solvent switch for LC-MS-MS, i.e. from laboratory sample to ready-to-inject solutions in autosampler vials.

\section{Quantification}

\section{$G C-M S$}

For each pesticide the concentrations were calculated for two diagnostic ions. In previous validation work (not published) using the same software it was found that for most pesticides automatic integration and repeatability of response were better when peak height, rather than area, was used. Peak height was therefore used, with few exceptions (e.g. pesticides prone to tailing, for example 2-phenylphenol). All responses were normalized to the response of the internal standard (antor). One-point calibration was performed using a fixed matrixmatched standard (tomato, see Results and discussion section) at a level corresponding to five times the LOQ. The linearity of the plot of MS response against concentration was verified periodically over the range 0.01 to $1-5 \mathrm{mg} \mathrm{kg}^{-1}$. For most pesticides linearity was adequate (relative response within $20 \%$ of the calibration standard) up to at least $1 \mathrm{mg} \mathrm{kg}^{-1}$.

\section{$L C-M S-M S$}

The internal standard (antor) was evaluated qualitatively only to confirm injection of the sample extract. Because of unpredictable and varying matrix effects for several of the matrices included in this work, normalization against the internal standard was not considered feasible. For each sample matrix that was fortified, a matrix-matched standard was also prepared by spiking the final extract of the corresponding control sample. Peak area was used for quantification. One-point calibration was performed using the matrix-matched standard at a level corresponding to five times the LOQ. Linearity of the MS response against concentration was verified periodically over the range 0.01 to $1 \mathrm{mg} \mathrm{kg}^{-1}$. For most pesticides, the relationship was linear (relative response within $20 \%$ of the calibration standard) up to at least $0.5 \mathrm{mg} \mathrm{kg}^{-1}$.

\section{Validation}

Initial method validation was performed in accordance with EU guidelines [36, 37]. Two times five portions of the ho- mogenized sample were spiked with a mixture of pesticides at a low level $\left(0.01 \mathrm{mg} \mathrm{kg}^{-1}\right.$ or lower) and at a level ten times higher. Together with two unfortified control portions of the sample, they were processed and analyzed as outlined above.

Additional method-performance data were acquired by analyzing fortified samples concurrently with each batch of samples. The spike level ( $0.05 \mathrm{mg} \mathrm{kg}^{-1}$ for most pesticides) was five times the LOQ. With each batch different products were selected as much as possible. In the compilation the emphasis was on products which are less frequently reported in the literature to challenge the applicability of the method as a "multi-matrix method". For this purpose samples were not pre-screened for absence of pesticides and, consequently, occasionally recoveries could not be determined, because of the relatively high levels incurred. Such results were eliminated from the data set.

Spectrophotometric measurement of removal of chlorophyll

For evaluation of the removal of chlorophyll by GCB and comparison with GPC, a lettuce extract was prepared by extracting $25 \mathrm{~g}$ lettuce with $40 \mathrm{~mL}$ ethyl acetate after addition of $25 \mathrm{~g}$ anhydrous sodium sulfate. As a reference, $0.8 \mathrm{~mL}$ ethyl acetate was added to $3.2 \mathrm{~mL}$ of this extract to bring the extract concentration to $0.5 \mathrm{~g} \mathrm{~mL}^{-1}$. For dispersive SPE, $100 \mathrm{mg}$ GCB was added to sets of duplicate tubes and $3.2 \mathrm{~mL}$ extract was added to all tubes. Solvent was then added to four sets of tubes: set one $0.8 \mathrm{~mL}$ ethyl acetate, set two $0.4 \mathrm{~mL}$ ethyl acetate and $0.4 \mathrm{~mL}$ toluene (i.e. $10 \%$ toluene), set three $0.8 \mathrm{~mL}$ toluene (20\% toluene), and set four $0.8 \mathrm{~mL}$ xylene ( $20 \%$ xylene). The extracts were vortex mixed and centrifuged.

For GPC clean-up, $2.5 \mathrm{~mL}$ lettuce extract was injected on to a $40 \mathrm{~cm} \times 28 \mathrm{~mm}$ i.d. Biobeads SX3 column with 1:1 ethyl acetate-cyclohexane as eluent. The fraction collected was such that at least $50 \%$ of the pyrethroids were recovered (fraction from 105-200 $\mathrm{mL}$ ). The eluate was first concentrated, by rotary evaporation at $40^{\circ} \mathrm{C}$, to approximately $5 \mathrm{~mL}$, then transferred to a tube for further concentration, under nitrogen gas, to $2.5 \mathrm{~mL}$.

Final extract concentration before and after clean-up was always $0.5 \mathrm{~g} \mathrm{~mL}^{-1}$. Aliquots of the extracts were transferred to a cuvet for spectrophotometric analysis at $450 \mathrm{~nm}$. If required, the extracts were diluted with ethyl acetate to bring absorption within the linear range. The amount of chlorophyll in the uncleaned extract was defined as $100 \%$. For calibration purposes the uncleaned extract was diluted $10,20,40,50$ and 100 times with ethyl acetate and a calibration plot was constructed. Chlorophyll remaining after clean-up was determined from the decrease in absorption at $450 \mathrm{~nm}$ compared with the absorption of the uncleaned lettuce extract. 


\section{Results and discussion}

Monitoring of residues in fresh produce for the food industry, especially trade and retail, calls for rapid turnaround, preferably within one or two days. This means sample preparation must be rapid and straightforward. With regard to cost and waste, consumption of solvents and reagents should be low. At the same time, EU directives with regard to sample definition (90/642/EEC, [34]) and laboratory sample size (2002/63/EC [35]) for residue analysis should be respected. This means, for example, that that a total of $2 \mathrm{~kg}$ grapes (after removal of stalks), five whole melons, or $1 \mathrm{~kg}$ strawberries (after removal of caps) must be processed. The actual analysis is performed on a subsample of the laboratory sample, after appropriate comminution. The more thorough the comminution, the smaller the subsample can be and the lower the amount of solvent needed for extraction. It has, furthermore, been reported that for well homogenized samples extraction by vortex mixing or shaking, instead of high-speed blending (Turrax) suffices for effective extraction [29], although there is still some debate on this matter [38].

\section{Homogenization}

For homogenization there are several possibilities. Food choppers or kitchen blenders are often used. Very thorough homogenization can be achieved with the latter, but it is not possible to process the entire laboratory sample at once. For this reason, large-scale food choppers are more suited. With such devices, homogeneity is not always optimum, as can be observed with, e.g., tomatoes, for which small pieces of skin drift in the "soup" obtained after homogenization. Subsampling of very small amounts is, therefore, not acceptable after this procedure, because the subsample would be insufficiently representative of the original sample. More thorough homogenization can be achieved after addition of dry-ice or liquid nitrogen (cryogenic homogenization). This procedure is recommended when reducing the subsample for analysis to $10 \mathrm{~g}$. This procedure is more laborious, however, because it involves cutting the sample into pieces, freezing the sample (usually overnight), cryogenic comminution, then dissipation of the dry-ice or liquid nitrogen before further processing or storage. It also puts higher demands on the cutter (blades) and requires additional precautions for the operators (protection against low temperatures and noise). Cryogenic comminution has been recommended for some pesticides because it reduces their degradation during this step [39].

In recent years the food trade and retail have been intensifying their residue-monitoring programs and require analytical data before harvest, before accepting an assign- ment, or before releasing their products from distribution centers to supermarkets. For fresh produce this means there is a much pressure on laboratories for rapid turnaround (24 $48 \mathrm{~h}$ ). This is difficult to achieve when the analysis involves overnight freezing for cryogenic comminution. Thus, for reasons of ease and speed, it was decided to retain the current procedure - ambient homogenization of the entire laboratory sample by use of a large scale food cutter (thus accepting the consequence that for a limited number of pesticides the concentration found might be an underestimate). Because of non-optimum homogenization with the food cutter, subsamples should not be too small, and further comminution is required for efficient extraction of systemic pesticides. This can be achieved during extraction by use of an Ultra Turrax. We have previously established the minimum size of subsample that did not negatively affect the repeatability of the analysis. This was done with samples which contained residues. For subsamples $(n=7)$ of 50 and $25 \mathrm{~g}$, the relative standard deviation (RSD\%) was below $8 \%$ for several pesticide-matrix combinations. For pear leaves (regarded as a difficult matrix to homogenize) containing bromopropylate, phosalone, and tolylfluanide it was observed that the RSD increased from $<8 \%$ to $14-18 \%$ when the amount of subsample was reduced from $25 \mathrm{~g}$ to $12.5 \mathrm{~g}$. From this it was concluded that, with our procedure, $25 \mathrm{~g}$ was the minimum required amount of subsample.

$\mathrm{pH}$ adjustment

In the ethyl acetate-extraction procedure analytes are extracted and partitioned between water (from the matrix itself, or added water for dry crops) and ethyl acetate in one step. For basic and acidic compounds the partitioning can be affected by $\mathrm{pH}$, which can vary substantially with the matrix. Because the same extract is to be used not only for GC-MS but also for LC-MS-MS (after changing the solvent to methanol) which, preferably, should also include analysis of basic and acidic pesticides, control of $\mathrm{pH}$ was regarded as necessary. A pH of approximately 6 was chosen as compromise for efficient extraction of basic and acidic compounds. Although acidic pesticides were not included in this work, data in the literature (for barley without $\mathrm{pH}$ adjustment, i.e. non-acidic conditions [26]) indicate they are extracted into ethyl acetate.

For $\mathrm{pH}$ adjustment others have used sodium hydroxide [16-18] or sodium hydrogen carbonate [11, 14, 25] (Table 1). A disadvantage of this is that the amount of salt needed depends on the acidity of the sample. Addition of too much will result in a high $\mathrm{pH}$ and possible degradation of basesensitive pesticides. To keep the method as straightforward as possible the $\mathrm{pH}$ was adjusted using a solution of concentrated phosphate buffer $\left(4 \mathrm{~mol} \mathrm{~L}^{-1}, 2 \mathrm{~mL}\right)$. A solution 
was preferred over addition of solid salts because this enabled use of a dispenser and eliminated additional weighing of the salts. The buffer resulted in appropriate $\mathrm{pH}$ adjustment for most matrices, although there were exceptions, for example lemon and lime.

\section{Extraction}

The two conditions most relevant to extraction efficiency are the sample-to-solvent ratio and addition of salt, which in ethyl acetate-based multi-residue methods has always been sodium sulfate.

The amount of ethyl acetate (in $\mathrm{mL}$ ) relative to the amount of sample (in $\mathrm{g}$ ) is, typically, at least $2: 1$. This ratio has been used for many years (Table 1). It results in good extraction efficiency and is practical with regard to achieving phase separation and avoidance of emulsions. To avoid sacrificing decades of method history no attempts were made to reduce the ratio; to do so might also adversely affect recovery and/or complicate phase separation. Larger amounts (as used by several other laboratories; Table 1) result in greater solvent consumption and more dilute extracts. In previous work [15] it has been shown that the efficiency of extraction of polar pesticides improves with the amount of salt added. When $50 \mathrm{~mL}$ ethyl acetate and $25 \mathrm{~g}$ sample were used, $25 \mathrm{~g}$ sodium sulfate was sufficient to obtain recoveries of $80 \%$ or better, even for very polar and highly water-soluble compounds, for example acephate and methamidophos. Because these recoveries were obtained with a single extraction it was found unnecessary to perform repeated extraction, as some laboratories are doing [11, 18, 20, 21]. For addition of the sodium sulfate an automatic salt-dispenser coupled to a balance, as is used in our laboratory, or a scoop, was found to be very convenient.

The extraction procedure involves successive addition of buffer, extraction solution (ethyl acetate with internal standard), and sodium sulfate to the centrifuge tube containing the sample, after which the pesticides are extracted and partitioned in one step using a Turrax. During this step the subsample is further comminuted for efficient extraction of the pesticides from the matrix. Vortex mixing, shaking or sonication were regarded as less efficient for subsamples that were homogenized in a large-scale food cutter under ambient conditions, but this was not investigated, partly because a variety of samples containing residues would be required to do so in an appropriate manner.

It was noted from the literature that filtration is often performed to separate the solid pellet from the liquid. Again, there is no real need for this step, which involves additional glassware and, occasionally, rinsing (diluting) of the extract. For many samples a clear ethyl acetate extract is obtained after settling; if not the tubes can be centrifuged.
This is no more laborious than filtration and does not involve additional glassware.

Because the same Turrax is used for several samples, carry-over is an aspect to be considered. Between samples the Turrax is cleaned first by rinsing with water, by means of a flow-through beaker, then by brief immersing in two beakers containing ethyl acetate. Using this procedure, carry-over was tested by analyzing a blank after a sample that had been fortified at $5 \mathrm{mg} \mathrm{kg}^{-1}$. Carry-over was less then $0.1 \%$, indicating that the straightforward cleaning procedure was sufficient to avoid cross-contamination up to $5 \mathrm{mg} \mathrm{kg}{ }^{-1}$ when setting reporting limits not lower than $0.01 \mathrm{mg} \mathrm{kg}^{-1}$.

\section{GC-MS analysis}

\section{Clean-up}

In ethyl acetate-based multiresidue methods either no cleanup or GPC clean-up is performed. This has hardly changed over the years (Table 1). In contrast with acetone and acetonitrile-based methods, in which SPE is commonly employed, this has been reported only occasionally for ethyl acetate-based methods. Obana et al. [10] used a cartridge packed with layers of water-absorbing polymer and GCB. Sharif et al. [21] described a clean-up using SAX/PSA but the scope of the method was restricted to organochlorine and organophosphorus pesticides. Zhang et al. [20] used a clean-up based on Florisil and achieved adequate recovery of many pesticides but not the more polar organophosphorus pesticides. It has been stated that in $\mathrm{GC}$ analysis with use of highly selective detectors, for example MS-MS no clean-up is required, even when injecting $15 \mathrm{mg}$ equivalent of matrix (green bean, tomato, pepper, cucumber, marrow, egg plant, and water melon [40]). Other laboratories experienced problems with contamination of the GC inlet and tried to solve this by automatic exchange of liner inserts $[14,41]$. This is in agreement with our experience that injection of $10 \mathrm{mg}$ matrix equivalent, especially for leafy vegetables, does result in rapid deterioration of system performance because of accumulation of non-volatile material in the inlet. This makes the system less robust, and frequent exchange of the liner (daily) and GC-pre column (weekly) is required. Another problem encountered with injection of the uncleaned extracts was a shift in the retention times of pesticides relative to that of the calibration standard for some sample extracts. This shift was insufficiently corrected by automatic adjustment of retention times relative to that of the internal standard. Typically, shifts were in the range $0.05-0.20 \mathrm{~min}$ and were most abundant for the "azole" pesticides. Such shifts can complicate automatic peak assignment during data-handling. When data acquisition is performed in a non-continuous mode (e.g. selected-ion 
monitoring or MS-MS) such shifts also increase the risk of pesticides shifting from their acquisition window. For injection of relatively large amounts of matrix (e.g. $10 \mathrm{mg}$ ) in GC analysis clean-up for removal of bulk co-extractants is therefore regarded as a prerequisite for robust analysis of a wide variety of vegetable and fruit matrices.

For vegetables and fruit matrices, chlorophyll (MW 900) and other pigments, for example carotenoids (e.g. $\beta$ carotene, MW 537) are typical bulk co-extractants. Most of these compounds are of low volatility and are not apparent as interferences in the chromatograms; they do, however, accumulate in the liner of the GC and eventually have an adverse effect on transfer of analytes to the column and/or on peak shape. Because of its high molecular weight, chlorophyll can be removed by GPC. A disadvantage is that the extract is strongly diluted and reconcentration by rotary evaporation is almost inevitable when LODs of $0.01 \mathrm{mg} \mathrm{kg}^{-1}$ are required. Such a step would contribute substantially to overall sample-preparation time. Although a very efficient on-line combination of GPC and GC-MS was described recently [42], avoiding GPC whenever possible would be even more straightforward. Solid-phase extraction is an alternative clean-up procedure which involves less dilution and is less laborious. Even more efficient is SPE in the socalled dispersive mode, as described by Anastassiades et al. [29]. Here the solid phase is simply added to the extract, thereby avoiding typical SPE procedures such as conditioning, sample transfer, elution, and evaporative reconcentration. The pesticides partition between the solid phase and the solvent and after vortex mixing and centrifugation the supernatant is ready for analysis.

Two stationary phases, graphitized carbon black (GCB) and phases with amino functionality, have been shown to be particularly effective for removing co-extracted material from the raw extract while not removing most of the pesticides; this makes them very suitable for wide-scope methods [28, 29, 31, 38, 43-45].

Initially, a method was envisaged using SPE column clean-up with GCB, because for leafy vegetables this was found to be the only sufficiently effective alternative to GPC. After the publication on dispersive SPE [29] it was decided to investigate this approach, thus sacrificing some clean-up potential (as has been reported in the literature [31]) for ease and speed.

GCB is well known to adsorb planar molecules, including chlorophyll and other pigments but also pesticides with planar functionality. In acetonitrile-based methods, toluene (typically $25 \%$ ) is often added to the eluent to desorb these pesticides also from the SPE column [28, 38, $43,45]$. One of the objectives of this work was to investigate the possibility of using GCB in a dispersive cleanup step without unacceptable losses of planar pesticides. First we investigated which pesticides, dissolved in ethyl acetate, are adsorbed by GCB. A somewhat arbitrary, $25 \mathrm{mg} \mathrm{mL}^{-1} \mathrm{GCB}$ phase was added to standard solutions. After vortex mixing and centrifugation the solution was analyzed by GC-MS (165 pesticides) and, after changing the solvent to methanol, by LC-MS-MS (another 70 pesticides), and the responses were compared with those from untreated standard solutions. For 35 pesticides (15\%) adsorption was observed (Table 2). In addition to the pesticides included in this test, it is known from the literature [44] that chinomethionate, furametpyr, and pyraclofos are also adsorbed by GCB (from acetone-cyclohexane, 1:4).

To investigate how much toluene is required to prevent adsorption of planar pesticides by GCB in dispersive SPE, the partitioning experiment was repeated with standard solutions of 10,20 , or $30 \%$ toluene in ethyl acetate. This was done for the GC-MS pesticide mixture only.

As is apparent from Fig. 1, even 10\% toluene dramatically improved recovery. With $20 \%$ toluene recovery of all pesticides was higher than $65 \%$. It should be noted that this experiment with standard solutions is the worst case. For real samples chlorophyll and carotenoids will also affect the distribution in favor of the pesticides in solution. Use of $30 \%$ of toluene further improved recovery only slightly. Twenty percent was regarded as optimum with regard to distribution and ease of solvent elimination in large-volume

Table 2 Pesticides adsorbed by $\mathrm{GCB}^{\mathrm{a}}$

\begin{tabular}{|c|c|c|}
\hline $\begin{array}{l}\text { Strong adsorption } \\
\text { (rec. 0-50\%) }\end{array}$ & $\begin{array}{l}\text { Medium adsorption } \\
\text { (rec. } 50-70 \% \text { ) }\end{array}$ & $\begin{array}{l}\text { Not } \\
\text { consistent }\end{array}$ \\
\hline \multicolumn{3}{|l|}{ Measured by GC-MS } \\
\hline Chlorothalonil & Azinphos-ethyl & Phosmet \\
\hline Cyprodinil & Azinphos-methyl & Prochloraz \\
\hline Fenazaquin & Chlorpyrifos-methyl & Pyrazophos \\
\hline Hexachlorobenzene & Dicloran & Trifluralin \\
\hline Mepanipyrim & EPN & \\
\hline Pentachloroaniline & Fenamiphos & \\
\hline Phosalone & Phorate & \\
\hline Pyrimethanil & Quintozene & \\
\hline Quinoxyfen & & \\
\hline \multicolumn{3}{|c|}{ Measured by LC-MS-MS } \\
\hline Carbendazim & Fenpyroximate & \\
\hline Clofentezine & Flufenoxuron & \\
\hline Desmedipham & Tricyclazole & \\
\hline Diflubenzuron & Triflumuron & \\
\hline Flucycloxuron & Thiophanate-methyl & \\
\hline \multicolumn{3}{|l|}{ Hexaflumuron } \\
\hline \multicolumn{3}{|l|}{ Phenmedipham } \\
\hline \multicolumn{3}{|l|}{ Pymetrozine } \\
\hline Thiabendazole & & \\
\hline
\end{tabular}

${ }^{\text {a }}$ Pesticides in ethyl acetate, $25 \mathrm{mg} \mathrm{GCB} \mathrm{mL}^{-1}$ solvent rec., recovered 
Fig. 1 Effect of the amount (\%) of toluene in ethyl acetate on recovery of pesticides adsorbed by GCB $\left(25 \mathrm{mg} \mathrm{mL}^{-1}\right) . h c b$, hexachlorobenzene; $p c a$, pentachloroaniline; ctn, chlorothalonil; mep, mepanipyrim; cypr, cyprodinil; pyri, pyrimethanil; fena, fenazaquin; quin, quinoxyfen; pyra, pyrazophos; epn, EPN

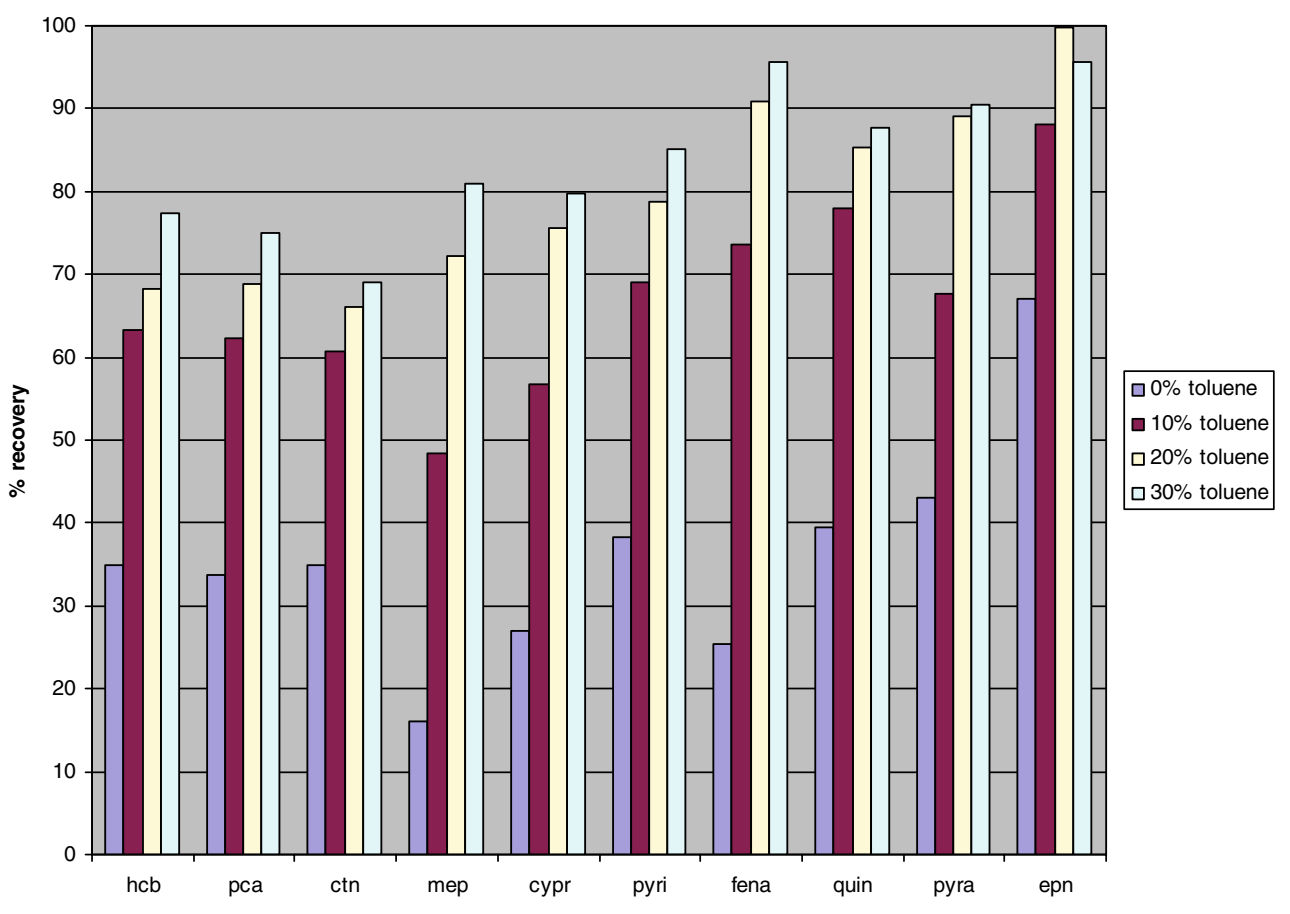

injection (see below). In addition to toluene, two alternative analogues, benzene and xylene, were also considered. Benzene, was not tested because it could not be used in routine practice because of its carcinogenic properties (although it would have been favorable with regard to solvent elimination). Xylene was tested in a similar way as toluene. Results obtained for hexachlorobenzene and chlorothalonil by use of the two solvents are compared in Fig. 2. Slight but consistently better recovery was obtained with xylene$>70 \%$ recovery could now be obtained for all pesticides. Because of its greater volatility, however, toluene was finally selected.

Obviously, toluene is also likely to affect adsorption of chlorophyll and/or carotenoids and might reduce the effectiveness of clean-up. To investigate this, a lettuce extract was prepared, the dispersive clean-up experiments were performed with different amounts of toluene, and removal of chlorophyll was verified. Visually it was clearly apparent that, despite addition of toluene, the intense green color turned light yellow, indicating that chlorophyll was removed to a large extent. To enable more quantitative evaluation, the extracts were also measured with a spectrophotometer at $450 \mathrm{~nm}$. For comparison, the same extracts were also cleaned by GPC. The results are presented in Table 3. Without toluene, chlorophyll was very effectively removed. Absorption at $450 \mathrm{~nm}$ was reduced by $94 \%$. Toluene, as expected, reduced adsorption of chlorophyll, but removal was still $87 \%$ or $78 \%$, after addition of $10 \%$ or $20 \%$ toluene in ethyl acetate, respectively. Similar to observations with the planar pesticides, adsorption was reduced slightly more by use of xylene than by use of toluene. With GPC, chlorophyll removal was $60 \%$. It should be noted here that the elution window was relatively wide, to include pyrethroids within the scope of the method. The elution windows for chlorophyll (and carotenoids) partially overlap those for pyrethroids, as has also been reported by others [44]. From


Fig. 2 Comparison of toluene and xylene as additives for preventing adsorption of planar pesticides by GCB in dispersive SPE 
Table 3 Removal of chlorophyll by dispersive SPE (GCB) and GPC

Clean-up procedure

Dispersive SPE, $100 \%$ ethyl acetate

Dispersive SPE, $10 \%$ toluene in ethyl acetate

Dispersive SPE, $20 \%$ toluene in ethyl acetate

Dispersive SPE, $20 \%$ xylene in ethyl acetate

GPC (fraction incl. pyrethroids)

Sample extract: lettuce $0.5 \mathrm{~g} \mathrm{~mL}^{-1}$. Dispersive SPE: $25 \mathrm{mg} \mathrm{GCB} \mathrm{mL} \mathrm{m}^{-1}$. GPC: wide scope elution window, i.e. including pyrethroids.

these experiments it can be concluded that chlorophyll has more affinity than the planar pesticides for GCB. In dispersive SPE toluene effectively prevents unacceptable adsorption of planar pesticides while to a large extent maintaining its cleaning properties in respect of chlorophyll. Dispersive GCB not only enables much faster chlorophyll removal, it is also more effective when including pyrethroids in the scope of the method. For non-fatty vegetable and/or fruit matrices, therefore, GPC is not required and dispersive GCB clean-up is a much faster alternative without sacrificing scope.

The GCB clean-up enabled continuous injection of extracts of leafy vegetables without rapid system deterioration. With some matrices, however (e.g. plums, grapefruit), retention time shifts were still observed. In addition, depending on the matrix, quite intensive interferences could be observed in the GC-MS TIC chromatograms. Further clean-up by PSA, complementing the GCB clean-up by removing compounds such as organic acids and sugars by hydrogen bonding, was therefore investigated. To keep sample clean-up as straightforward and rapid as possible focus was on a combined dispersive GCB/PSA clean-up.

After the outcome of the GCB experiments, partitioning of the pesticides and co-extractants will be between PSA and ethyl acetate-toluene, 8:2. Because no information was available about the distribution of pesticides between these two phases, this was obtained by analyzing pesticide standards in ethyl acetate-toluene, 8:2, with and without PSA. Preliminary experience with dispersive PSA clean-up revealed that with some matrices (e.g. cereals) $25 \mathrm{mg} \mathrm{mL}^{-1}$ did not result in complete elimination of interfering compounds (e.g. fatty acids) typically removed by PSA. Partitioning with a much larger amount of adsorbent (200 mg mL ${ }^{-1}$ ) was, therefore, also studied.

With $25 \mathrm{mg} \mathrm{mL}^{-1}$ losses of $30-40 \%$ were observed for sixteen pesticides, most probably as a result of adsorption, although the possibility of degradation induced by the basic nature of the PSA material could not be fully excluded. The findings were confirmed by the experiment with $200 \mathrm{mg}$ PSA $\mathrm{mL}^{-1}$ (Table 4). The pesticides for which interaction with PSA was observed all had a $\mathrm{C}=\mathrm{O}$ or $\mathrm{P}=\mathrm{O}$ group in common (except for chlorothalonil). Our findings are not in full agreement with those of Anastassiades et al. [29] who did not observe losses as a result of using PSA. For this there can be two explanations. In our experiment adsorption was tested with standard solution rather than matrix. Coextractants in matrix are likely to compete with the pesticides during adsorption. Second, with our method the organic phase (ethyl acetate-toluene, 8:2) is less polar than the acetonitrile phase; this could result in a stronger interaction between the polar functionality of the pesticides and amino functionality of PSA. From our results it became clear that with regard to the amount of PSA "the more, the better" does not apply. Another observation was that a hump appeared in the TIC chromatogram after a $20-\mu \mathrm{L}$ injection of solvent mixed with $200 \mathrm{mg}$ PSA $\mathrm{mL}^{-1}$. This hump, which eluted between 6 and $12 \mathrm{~min}$, consisted of many peaks and a variety of masses. Cleaning of the PSA by washing with ethyl acetate $(3 \times 20 \mathrm{~mL}$ for $1 \mathrm{~g})$, then drying by rotary evaporation, eliminated this contamination without affecting the clean-up properties. To keep the method straightforward, $25 \mathrm{mg}$ PSA $\mathrm{mL}^{-1}$ was used as default, and the material was not cleaned before use.

The clean-up proved effective at reducing retention time shifts. As an example, for a plum extract without clean-up, the retention times of 24 pesticides (out of 140) were shifted by more than $0.05 \mathrm{~min}$ compared with the calibration standard. After clean-up this occurred for three pesticides only. With other matrices also shifts were reduced, but for

Table 4 Adsorption of pesticides by PSA

\begin{tabular}{ll}
\hline Pesticide & Recovery (\%) \\
\hline Acephate & $43^{\mathrm{a}}$ \\
Acrinathrin & $41^{\mathrm{b}}$ \\
Asulam & $0^{\mathrm{a}}$ \\
Carbaryl & $56^{\mathrm{b}}$ \\
Chlorothalonil & $17^{\mathrm{b}}$ \\
Cycloxidim & $39^{\mathrm{a}}$ \\
Dichlorvos & $33^{\mathrm{b}}$ \\
Dimethoate & $62^{\mathrm{b}}$ \\
Hymexazol & $0^{\mathrm{a}}$ \\
Mevinphos & $62^{\mathrm{b}}$ \\
Phosmet & $25^{\mathrm{b}}$ \\
Phosphamidon & $63^{\mathrm{b}}$ \\
Profenofos & $56^{\mathrm{b}}$ \\
Pyridate & $40^{\mathrm{a}}$ \\
Pyridate-metabolite & $7^{\mathrm{a}}$ \\
Sethoxydim & $48^{\mathrm{a}}$ \\
\hline
\end{tabular}

${ }^{\mathrm{a}}$ After partitioning with ethyl acetate, $25 \mathrm{mg} \mathrm{mL}^{-1}$ and LC-MS-MS analysis

${ }^{\mathrm{b}}$ After partitioning with ethyl acetate-toluene, 8:2, $200 \mathrm{mg}$ PSA mL ${ }^{-1}$ and $\mathrm{GC}-\mathrm{MS}$ analysis 
some matrices (herbs, e.g. parsley) deviations were still quite common.

As an illustration of the removal of co-extractants from the ethyl acetate extract (or, in fact, from the ethyl acetatetoluene, 8:2, extract) by dispersive GCB/PSA clean-up, GC-MS total ion current chromatograms of extracts obtained with and without clean-up are shown in Fig. 3. The most apparent differences are indicated. Several abundant matrix peaks are removed or strongly reduced. For lettuce, the overall background level between 15 and 25 min was also reduced. This clearly visible clean-up was mainly caused by the PSA material. With GCB alone differences between cleaned and uncleaned were much less apparent. The main benefit of GCB was prevention of rapid build up of non-volatile material (chlorophyll) in the liner, which enables prolonged use of the system without maintenance. Experience with method for more than three years and analysis of over 15,000 vegetable and fruit samples shows that, on average, the liner must typically be replaced weekly (after 150-200 injections; iprodion, dimethipin, and chlorfenapyr are the first for which response is lost). Further GC-MS maintenance consists in replacement of pre-column once of twice a month. The GC column is replaced approximately twice a year. The source of the MS is cleaned once a month.

In a continuing search for even further simplification of sample preparation, the possibility of combined extraction and dispersive SPE clean-up in one step was investigated. For two matrices (lettuce and mandarin, fortified with 140 pesticides, triplicate experiments) the solid phase materials (GCB/PSA, relative amounts similar to previous experiments) were added directly to the centrifuge tube containing the sample, sodium sulfate, and the extraction solvent (to which 20\% toluene had been added). After Turrax extraction and centrifugation, the extract was ready for injection into the GC. Recovery was compared with that obtained by use of dispersive clean-up after separation of the ethyl acetate extract from the sample mixture. As could be seen from the color of the extract (the lettuce extract was almost colorless) the GCB remained effective. Adsorption of chlorophyll is based on planarity (shape) rather than polarity and, therefore, this will occur from both the aqueous and the organic phases. As was to be expected, the same was not true for PSA. The presence of water prevented adsorption of co-extractants with a hydroxyl group, i.e. almost identical GC-MS total-ion chromatograms were obtained from extracts which were not cleaned and from those cleaned in the centrifuge tube. Pesticide recovery obtained after use of successive or simultaneous dispersive SPE clean-up was very similar, although recovery of some pesticides in the combined approach was too high, because of co-elution of interferences. The final method therefore used successive extraction and dispersive SPE clean-up.

\section{Large-volume injection}

GC-MS analysis of sample extracts was performed in fullscan mode. This enables detection of any GC-amenable pesticide. Because system LOQ for a quadrupole mass spectrometer in full-scan mode is limited, conservatively estimated at $100 \mathrm{pg}, 10 \mathrm{mg}$ matrix equivalent must be introduced into the GC to reach a target LOQ of $0.01 \mathrm{mg}$ $\mathrm{kg}^{-1}$. With an extract concentration of $0.5 \mathrm{~g} \mathrm{~mL}^{-1}$, this means $20 \mu \mathrm{L}$ must be introduced into the GC. Off-line tenfold evaporative concentration and then $2 \mu \mathrm{L}$ injection could also be performed, but this would involve clean-up of larger volumes of extract, the risk of loss of the volatile pesticides (e.g. dichlorvos), and an additional step in sample preparation. Although large-volume injection in GC is a well established technique $[47,48]$, many routine laboratories are still reluctant to apply it; if they do, the volume is often restricted to $5-10 \mu \mathrm{L}$. Such volumes can be accommodated in liners with a frit or even in empty (baffled) liners when injection speed is carefully adjusted. For larger volumes there is a risk of flooding [46], i.e. that extract is lost as liquid through the split exit. To prevent this, liners can be packed with a variety of materials. Packing materials often have the disadvantage of a large surface area with active sites, however, resulting in degradation and/or adsorption of thermo labile and/or polar pesticides; problems can also be encountered with splitless transfer of higher boiling pesticides (e.g. deltamethrin) from the liner to the GC column. Other disadvantages can be a pressure drop over the liner (slows down solvent elimination) and liner-to-liner variability requiring re-optimization of the solvent-elimination process after liner replacement. A means of by-passing the disadvantages of packed liners while still achieving accommodation of $20-50 \mu \mathrm{L}$ of liquid was described in 1993 by Staniewski and Rijks [49]. They developed a liner with a sintered porous glass bed on the inner surface wall of the liner. The liquid is retained in the porous glass bed. The potentially active glass surface area is relatively small compared with the materials in packed liners. The gas flow is not obstructed, because the centre of the liner is empty. This enables efficient solvent vapor removal during solvent elimination and efficient transfer of analytes to the analytical column during splitless injection after solvent elimination. Since the early 2000s such liners have been commercially available for PTV injectors from several suppliers, and since then our laboratory has implemented $20 \mu \mathrm{L}$ as default injection volume for ethyl acetate.

After the development of the dispersive GCB clean-up, the solvent to be introduced into the GC contained $20 \%$ toluene, which might effect the processes involved in largevolume injection differently from $100 \%$ ethyl acetate. Because toluene does not evaporate azeotropically with ethyl acetate and is less volatile, it will be the main solvent left at 


\section{Sample ID: 13/test}

G081219

1007

1007
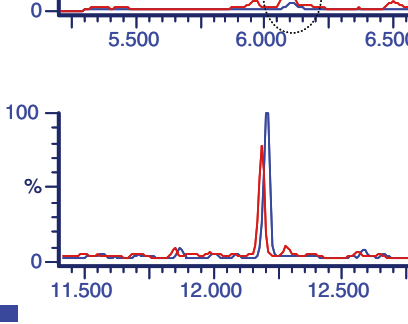

100
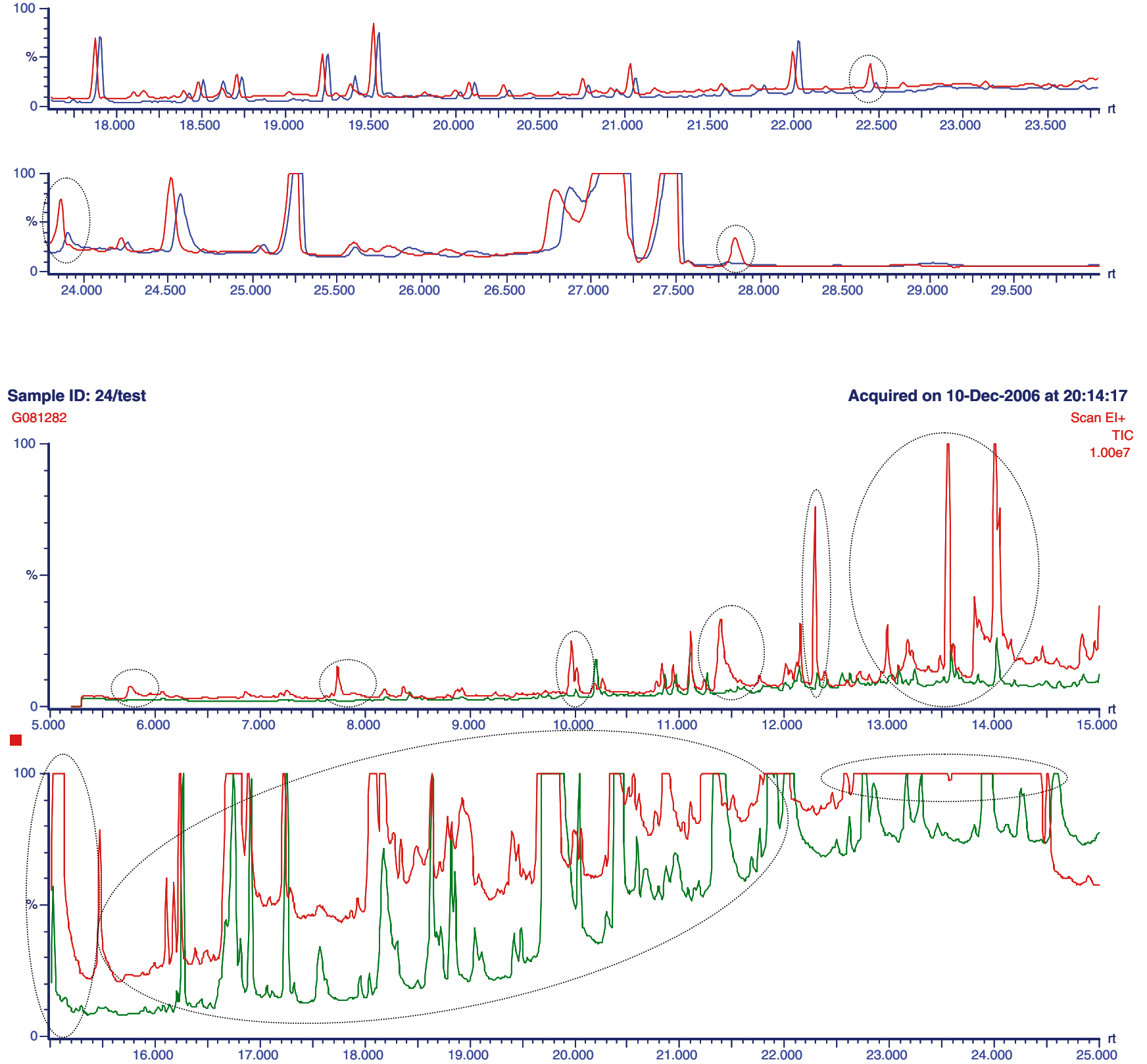

Fig. 3 GC-MS chromatograms. Overlay total ion chromatograms (TICs) obtained after $20 \mu \mathrm{L}$ injection of an extract of mandarin (top) and lettuce (bottom) without (higher peaks) and with clean-up 
the end of the evaporation process. Injection of $20 \mu \mathrm{L} 20 \%$ toluene in ethyl acetate means that $4 \mu \mathrm{L}$ toluene is introduced. The PTV used in this work was equipped with a $1 \mathrm{~mm}$ i.d. porous glass bed liner that could hold approximately $30 \mu \mathrm{L}$ within the zone that is appropriately heated during splitless transfer. Up to this volume there is no need for optimization of injection speed. To obtain information about splitless transfer of the last few microliters of toluene after solvent elimination, cold splitless injections of 1,2 , and $3 \mu \mathrm{L}$ of standards in $100 \%$ toluene were performed. Even with $2-\mu \mathrm{L}$ volumes peak distortion (fronting peak shape) was observed for pesticides of medium volatility. With $1 \mu \mathrm{L}$ injections peak shape was good and for several pesticides even better than for ethyl acetate. On injection of $20 \mu \mathrm{L}$ standard in ethyl acetatetoluene, $8: 2$, in the solvent-vent mode, no peak distortion was observed, indicating that less then $2 \mu \mathrm{L}$ toluene remained in the injector after the solvent-vent step. As observed earlier with large-volume injection of ethyl acetate, the vent time (here set at $40 \mathrm{~s}$ using an initial PTV temperature of $50^{\circ} \mathrm{C}$ ) was not at all critical, even for the most volatile pesticide (dichlorvos). Venting for 35 or $50 \mathrm{~s}$ did not dramatically affect responses or peak shape of the pesticides. In our experience, this phenomenon is typical for porous glass bed liners and contributes to the robustness of the method.

\section{Validation of $G C-M S$ method}

In the past a method based on simple ethyl acetate extraction followed by direct GC-MS analysis of the raw extract [4] had been validated for concentrations in the range 0.05-0.5 $\mathrm{mg} \mathrm{kg}{ }^{-1}$. The modified method described here involved a dispersive clean-up step, large-volume injection, and injection of ten times more matrix into the GC. Re-validation was therefore required, and focused on method performance at low concentrations. This was done using lettuce as matrix. The validation set consisted of two control samples, five fortifications at $0.001-0.05 \mathrm{mg} \mathrm{kg}^{-1}$ and five fortifications at a level ten times higher. Over 200 pesticides were included in the validation procedure. The results are presented in Table 5. For the $0.01-0.5 \mathrm{mg} \mathrm{kg}^{-1}$ concentration range the EU criteria (recovery $70-110 \%$, RSD $30 \%, 20 \%$, or $15 \%$ for $\leq 0.01,>0.01-0.1$, and $>0.1-1 \mathrm{mg}$ $\mathrm{kg}^{-1}$, respectively [37]) were met for 184 of the 201 pesticides included in the validation. At a level a factor of ten lower (fortification in the $0.001-0.01 \mathrm{mg} \mathrm{kg}^{-1}$ range for most pesticides) 147 pesticides could still be detected and for most $(78 \%)$ of these recovery and RSDs were acceptable. For many pesticides $S / N$ ratios were surprisingly good and background-corrected mass spectra often contained sufficient diagnostic ions (or were even recognizable mass spectra) to enable identity confirmation, as is illustrated in Fig. 4. The limits of detection, defined as $S / N=3$ for one favorable diagnostic ion for each pesticide, were determined on the basis of the signals from the low fortification levels and the average noise observed in duplicate control samples. The LOD was at or below $0.001 \mathrm{mg} \mathrm{kg}^{-1}$ for 78 pesticides, between 0.001 and $0.005 \mathrm{mg} \mathrm{kg}^{-1}$ for 73 pesticides, between 0.005 and $0.01 \mathrm{mg} \mathrm{kg}^{-1}$ for 29 pesticides, between 0.01 and $0.05 \mathrm{mg} \mathrm{kg}^{-1}$ for 16 pesticides, and higher for four pesticides.

This initial validation clearly showed it is possible to introduce $10 \mathrm{mg}$ of matrix equivalent of generic extracts obtained after ethyl acetate extraction of leafy vegetables. Adequate quantitative data are obtained for most of the pesticides at levels of $0.01 \mathrm{mg} \mathrm{kg}^{-1}$ or even below. Detection limits were usually well below $0.01 \mathrm{mg} \mathrm{kg}^{-1}$ after full-scan acquisition with a single-quadrupole MS. This means that for most pesticides at the target LOQ of $0.01 \mathrm{mg}$ $\mathrm{kg}^{-1}$ (i.e. the lowest maximum residue limit set in the EU for vegetables and fruit), the signal-to-noise ratio is adequate for reliable automatic integration of peaks and that confirmation of identity of the pesticide is possible from its mass spectrum or at least one or two other diagnostic ions.

Pesticides that did not meet the EU criteria for quantitative analysis, and/or for which relatively high LODs were obtained, included many compounds known to be troublesome in $\mathrm{GC}$ analysis because of to their high polarity or thermal lability. Typical examples are acephate, cyromazine, dicofol (screened for as its degradation product dichlorobenzophenone), dimethoate, imazalil, metaldehyde, methamidophos, methiocarb, omethoate, and the benzoylureas (measured as one common and one compound-specific degradation product). The relatively low recovery of the polar organophosphorus pesticides (acephate, methamidophos, and omethoate) can be attributed to the GC measurement and not to poor extraction efficiency, as was apparent from LC-MS-MS analysis of samples using the same extraction technique (see section $L C-M S-M S$ analysis). For several other polar or labile pesticides adequate quantitative data were obtained during this initial validation, but from previous experience and the results obtained after implementation of the method it was clear that for such compounds LC-based analysis is more robust than GC-MS analysis. Typical examples include carbaryl, carbofuran, clofentezin, monocrotophos, and oxydemeton-methyl.

\section{Analytical quality-control data from routine GC-MS analysis}

The initial validation data are continuously being supplemented by performance data generated as part of the analytical quality-control during routine analysis of the samples, to gain insight into reproducibility, robustness, 
Table 5 GC-MS re-validation data for pesticides in lettuce

\begin{tabular}{|c|c|c|c|c|c|c|c|c|c|c|}
\hline & Pesticide & $t_{\mathrm{R}}(\min )$ & $m / z$ (quant) & Level $\left(\mathrm{mg} \mathrm{kg}^{-1}\right)$ & Rec. (\%) & RSD (\%) & Level $\left(\mathrm{mg} \mathrm{kg}^{-1}\right)$ & Rec. $(\%)$ & RSD (\%) & $\operatorname{LOD}\left(\mathrm{mg} \mathrm{kg}^{-1}\right)$ \\
\hline 1 & Acephate & 10.45 & 136 & 0.026 & 35 & 4 & 0.257 & $\underline{58}$ & 9 & 0.006 \\
\hline 2 & Acrinathrin & 22.06 & 289 & 0.018 & $\overline{118}$ & 15 & 0.178 & $\overline{94}$ & 9 & 0.003 \\
\hline 3 & Aldrin & 16.58 & 265 & 0.003 & $\overline{139}$ & 25 & 0.031 & 94 & 2 & 0.002 \\
\hline 4 & Atrazine & 14.17 & 215 & 0.002 & $\overline{91}$ & 21 & 0.018 & 98 & 7 & 0.002 \\
\hline 5 & Azinphos-methyl & 21.64 & 160 & 0.01 & $\underline{119}$ & 9 & 0.098 & 110 & 7 & 0.009 \\
\hline 6 & Azoxystrobin & 25.80 & 344 & 0.01 & 82 & 8 & 0.099 & 92 & 5 & 0.003 \\
\hline 7 & Benalaxyl & 19.82 & 148 & 0.005 & 85 & 9 & 0.047 & 90 & 8 & 0.002 \\
\hline 8 & Benzoylurea (deg) ${ }^{a}$ & 8.90 & 141 & & $\underline{113}$ & 5 & 0.025 & 110 & 6 & \\
\hline 9 & Bifenthrin & 20.91 & 181 & 0.007 & 84 & 9 & 0.068 & 89 & 13 & $\leq 0.001$ \\
\hline 10 & Biphenyl & 9.81 & 154 & 0.006 & 97 & 10 & 0.063 & 101 & 5 & $\leq 0.001$ \\
\hline 11 & Bitertanol & 22.89 & 170 & 0.003 & 83 & 9 & 0.031 & 90 & 4 & 0.002 \\
\hline 12 & Bromophos & 17.02 & 331 & 0.003 & 99 & 7 & 0.032 & 105 & 2 & $\leq 0.001$ \\
\hline 13 & Bromopropylate & 20.94 & 343 & 0.003 & 103 & 13 & 0.032 & 89 & 5 & 0.001 \\
\hline 14 & Bromuconazole & 20.86 & 173 & 0.002 & 109 & 12 & 0.024 & 91 & 6 & $\leq 0.001$ \\
\hline 15 & Bupirimate & 18.72 & 273 & 0.003 & $\underline{61}$ & 8 & 0.032 & 91 & 5 & 0.001 \\
\hline 16 & Buprofezin & 18.68 & 172 & 0.002 & $\overline{85}$ & 14 & 0.019 & 92 & 8 & 0.001 \\
\hline 17 & Cadusafos & 13.46 & 158 & 0.002 & $\underline{117}$ & 18 & 0.021 & 92 & 11 & 0.001 \\
\hline 18 & Carbaryl & 15.84 & 115 & 0.004 & $\overline{93}$ & 9 & 0.04 & 93 & 8 & 0.002 \\
\hline 19 & Carbofuran & 14.10 & 164 & 0.003 & 88 & 7 & 0.033 & 93 & 3 & 0.002 \\
\hline 20 & Chlordane, alpha- & 17.81 & 373 & 0.001 & * & * & 0.015 & 92 & 4 & 0.002 \\
\hline 21 & Chlordane, gamma- & 18.12 & 373 & 0.002 & 84 & 7 & 0.015 & 96 & 4 & 0.001 \\
\hline 22 & Chlorfenvinphos & 17.47 & 323 & 0.003 & 84 & 6 & 0.03 & 97 & 5 & 0.001 \\
\hline 23 & Chloroaniline, 3- & 7.49 & 127 & 0.002 & $*$ & $*$ & 0.025 & $\underline{25}$ & $\underline{46}$ & 0.003 \\
\hline 24 & Chlorobenzilate & 19.10 & 251 & 0.005 & * & * & 0.05 & $\overline{95}$ & $\overline{4}$ & 0.010 \\
\hline 25 & Chlorothalonil & 15.05 & 264 & 0.004 & 146 & 15 & 0.042 & 136 & 9 & $\leq 0.001$ \\
\hline 26 & Chlorpropham & 13.08 & 171 & 0.006 & $\bar{*}$ & $*$ & 0.059 & $\overline{95}$ & 6 & 0.015 \\
\hline 27 & Chlorpyrifos & 16.67 & 314 & 0.003 & 102 & 16 & 0.034 & 102 & 5 & 0.002 \\
\hline 28 & Chlorpyrifos-methyl & 15.70 & 286 & 0.001 & 105 & 5 & 0.015 & 102 & 6 & $\leq 0.001$ \\
\hline 29 & Chlorthal-dimethyl & 16.77 & 301 & 0.005 & 90 & 7 & 0.051 & 91 & 4 & 0.001 \\
\hline 30 & Cinerin-1 & 18.67 & 150 & 0.053 & 84 & 3 & 0.528 & 93 & 6 & 0.041 \\
\hline 31 & Clofentezine & 22.45 & 304 & 0.014 & $*$ & * & 0.14 & 101 & 14 & 0.050 \\
\hline 32 & Cyfluthrin I & 23.33 & 226 & 0.041 & 91 & 7 & 0.407 & 93 & 6 & 0.023 \\
\hline 33 & Cyfluthrin II & 23.60 & 226 & 0.041 & 100 & 8 & 0.407 & 88 & 8 & 0.016 \\
\hline 34 & Cyhalothrin-lambda & 21.91 & 181 & 0.003 & 110 & 10 & 0.029 & 93 & 6 & 0.002 \\
\hline 35 & Cypermethrin-I & 23.65 & 163 & 0.018 & 107 & $\underline{29}$ & 0.184 & 96 & 5 & 0.008 \\
\hline 36 & Cypermethrin-II & 23.83 & 181 & 0.018 & 94 & $\overline{16}$ & 0.184 & 97 & 5 & 0.006 \\
\hline 37 & Cypermethrin-III & 24.07 & 181 & 0.018 & 96 & 10 & 0.184 & 96 & 6 & 0.013 \\
\hline 38 & Cyproconazole & 18.97 & 222 & 0.006 & 72 & 20 & 0.059 & 88 & 7 & 0.001 \\
\hline 39 & Cyprodinyl & 17.19 & 224 & 0.005 & 105 & 25 & 0.051 & 85 & 10 & $\leq 0.001$ \\
\hline 40 & Cyromazine & 14.47 & 166 & 0.013 & * & * & 0.13 & 82 & $\underline{56}$ & 0.040 \\
\hline 41 & DDE, $o, p^{\prime}-$ & 17.90 & 248 & 0.002 & * & * & 0.015 & 92 & 3 & 0.009 \\
\hline 42 & DDE, $p, p^{\prime}-$ & 18.50 & 248 & 0.001 & 110 & 11 & 0.015 & 100 & 5 & $\leq 0.001$ \\
\hline 43 & $\mathrm{DDT}, o, p^{\prime}-$ & 19.32 & 235 & 0.001 & 102 & 9 & 0.015 & 94 & 7 & 0.001 \\
\hline 44 & DDT, $p, p^{\prime}-$ & 20.28 & 235 & 0.002 & 86 & 11 & 0.016 & 95 & 8 & 0.001 \\
\hline 45 & Deltamethrin & 25.44 & 253 & 0.022 & 114 & 9 & 0.223 & 106 & 5 & 0.014 \\
\hline 46 & Demeton-S-methyl-sulfone & 16.11 & 169 & 0.03 & $\overline{71}$ & 15 & 0.302 & 91 & 9 & 0.004 \\
\hline 47 & Desmethylpirimicarb & 15.42 & 152 & 0.003 & * & * & 0.026 & 76 & 7 & 0.005 \\
\hline 48 & Diazinon & 14.70 & 137 & 0.002 & 98 & 14 & 0.019 & 94 & 3 & 0.001 \\
\hline 49 & Dichlofluanid & 16.41 & 224 & 0.004 & 79 & 9 & 0.044 & 98 & 8 & $\leq 0.001$ \\
\hline 50 & Dichlorvos & 8.00 & 185 & 0.002 & 107 & 6 & 0.018 & 92 & 7 & $\leq 0.001$ \\
\hline 51 & Dicloran & 13.96 & 206 & 0.003 & 96 & 16 & 0.029 & 106 & 2 & 0.003 \\
\hline 52 & Dicofol (as DCBP) & 16.75 & 250 & 0.005 & $*$ & $*$ & 0.049 & $\underline{126}$ & $\underline{33}$ & 0.010 \\
\hline 53 & Dieldrin & 18.56 & 263 & 0.004 & * & * & 0.041 & $\overline{95}$ & $\overline{6}$ & 0.005 \\
\hline 54 & Diethofencarb & 16.53 & 267 & 0.005 & 98 & 5 & 0.046 & 96 & 6 & 0.001 \\
\hline 55 & Difenoconazole-I & 25.12 & 323 & 0.029 & 94 & 10 & 0.288 & 95 & 3 & 0.006 \\
\hline 56 & Difenoconazole-II & 25.36 & 323 & 0.029 & 91 & 9 & 0.288 & 99 & 3 & 0.003 \\
\hline
\end{tabular}


Table 5 (continued)

\begin{tabular}{|c|c|c|c|c|c|c|c|c|c|c|}
\hline & Pesticide & $t_{\mathrm{R}}(\min )$ & $m / z$ (quant) & Level $\left(\mathrm{mg} \mathrm{kg}^{-1}\right)$ & Rec. (\%) & RSD (\%) & Level $\left(\mathrm{mg} \mathrm{kg}^{-1}\right)$ & Rec. (\%) & RSD (\%) & $\mathrm{LOD}\left(\mathrm{mg} \mathrm{kg}^{-1}\right)$ \\
\hline 57 & Diflubenzuron (deg) & 6.63 & 153 & 0.005 & 124 & 9 & 0.05 & 107 & 2 & 0.002 \\
\hline 58 & Dimethoate & 13.97 & 125 & 0.009 & $*$ & $*$ & 0.091 & 91 & 4 & 0.017 \\
\hline 59 & Dimethomorph & 25.88 & 301 & 0.021 & 95 & 7 & 0.207 & 87 & 5 & 0.002 \\
\hline 60 & Diniconazole & 19.54 & 268 & 0.002 & $*$ & $*$ & 0.018 & 89 & 12 & 0.003 \\
\hline 61 & Diphenylamine & 12.76 & 169 & 0.003 & 86 & 10 & 0.028 & 72 & 15 & $\leq 0.001$ \\
\hline 62 & Disulfoton & 14.81 & 88 & 0.005 & 101 & 5 & 0.05 & 96 & 3 & 0.002 \\
\hline 63 & DMSA & 13.19 & 200 & 0.005 & 87 & 9 & 0.052 & 92 & 7 & 0.002 \\
\hline 64 & DMST & 14.37 & 214 & 0.005 & * & * & 0.053 & 73 & 32 & 0.019 \\
\hline 65 & Dodemorph & 16.95 & 154 & 0.005 & $\underline{67}$ & 26 & 0.046 & 91 & $\overline{7}$ & 0.002 \\
\hline 66 & Edifenfos & 18.07 & 310 & 0.005 & $\overline{96}$ & 10 & 0.05 & 94 & 8 & 0.001 \\
\hline 67 & Endosulfan-alpha & 18.08 & $239+197$ & 0.005 & $*$ & $*$ & 0.047 & 93 & 5 & 0.010 \\
\hline 68 & Endosulfan-beta & 19.19 & $195+241$ & 0.005 & $*$ & $*$ & 0.046 & 87 & 1 & 0.020 \\
\hline 69 & Endosulfan-sulfate & 19.98 & $274+237$ & 0.005 & 82 & 10 & 0.047 & 97 & 4 & 0.004 \\
\hline 70 & Endrin & 20.94 & 245 & 0.005 & $*$ & $*$ & 0.051 & 90 & 8 & 0.006 \\
\hline 71 & EPN & 20.57 & 169 & 0.01 & 103 & 23 & 0.099 & 94 & 7 & 0.001 \\
\hline 72 & Epoxiconazole & 20.55 & 194 & 0.007 & $*$ & $*$ & 0.066 & 92 & 1 & 0.010 \\
\hline 73 & Esfenvalerate & 24.77 & 125 & 0.004 & $*$ & $*$ & 0.036 & 98 & 5 & 0.008 \\
\hline 74 & Ethion & 19.36 & 231 & 0.003 & * & $*$ & 0.03 & 97 & 3 & 0.007 \\
\hline 75 & Ethoprofos & 12.86 & 158 & 0.003 & 88 & 17 & 0.026 & 93 & 5 & 0.001 \\
\hline 76 & Etofenprox & 23.85 & 164 & 0.005 & 100 & 11 & 0.049 & 93 & 5 & 0.004 \\
\hline 77 & Etridiazole & 10.74 & 211 & 0.014 & 95 & 8 & 0.138 & 98 & 4 & 0.001 \\
\hline 78 & Etrimfos & 15.01 & 292 & 0.003 & 96 & 4 & 0.025 & 93 & 5 & $\leq 0.001$ \\
\hline 79 & Famoxadone & 25.90 & 330 & 0.01 & 97 & 9 & 0.1 & 96 & 5 & 0.003 \\
\hline 80 & Fenamiphos & 18.23 & 303 & 0.015 & 97 & 6 & 0.154 & 91 & 11 & $\leq 0.001$ \\
\hline 81 & Fenarimol & 22.13 & 139 & 0.004 & $*$ & $*$ & 0.038 & 101 & 4 & 0.008 \\
\hline 82 & Fenazaquin & 21.22 & 160 & 0.003 & $\underline{152}$ & 12 & 0.027 & 114 & 8 & 0.001 \\
\hline 83 & Fenbuconazole & 23.30 & 129 & 0.003 & $\bar{*}$ & $*$ & 0.03 & $\overline{92}$ & 3 & 0.006 \\
\hline 84 & Fenhexamid & 20.10 & 177 & 0.003 & $*$ & $*$ & 0.026 & 90 & 7 & 0.004 \\
\hline 85 & Fenitrothion & 16.25 & 260 & 0.001 & $*$ & $*$ & 0.015 & 95 & 8 & 0.003 \\
\hline 86 & Fenoxycarb & 20.89 & 116 & 0.015 & $\underline{117}$ & 8 & 0.154 & 94 & 4 & 0.002 \\
\hline 87 & Fenpiclonil & 20.78 & 238 & 0.007 & 88 & 5 & 0.071 & 92 & 8 & 0.003 \\
\hline 88 & Fenpropathrin & 21.05 & 181 & 0.005 & 77 & 13 & 0.05 & 92 & 13 & 0.001 \\
\hline 89 & Fenpropimorph & 16.63 & 128 & 0.001 & $*$ & * & 0.01 & 93 & 2 & 0.002 \\
\hline 90 & Fenthion & 16.63 & 278 & 0.002 & 99 & 7 & 0.023 & 99 & 5 & $\leq 0.001$ \\
\hline 91 & Fenvalerate & 24.54 & 167 & 0.004 & $*$ & $*$ & 0.036 & 103 & 8 & 0.006 \\
\hline 92 & Fipronil & 17.57 & 367 & 0.002 & 81 & 6 & 0.024 & 94 & 9 & $\leq 0.001$ \\
\hline 93 & Flucythrinate-I & 23.77 & 199 & 0.017 & 93 & 11 & 0.174 & 92 & 1 & 0.004 \\
\hline 94 & Flucythrinate-II & 18.51 & 199 & 0.017 & 94 & 6 & 0.174 & 93 & 4 & 0.004 \\
\hline 95 & Fludioxonil & 19.05 & 248 & 0.003 & $\underline{113}$ & 13 & 0.027 & 97 & 3 & 0.001 \\
\hline 96 & Flufenoxuron (deg) & 14.79 & 331 & 0.012 & $\overline{104}$ & 13 & 0.118 & $\underline{118}$ & 19 & 0.005 \\
\hline 97 & Flusilazole & 18.70 & 233 & 0.006 & 68 & 8 & 0.055 & $\overline{87}$ & 6 & $\leq 0.001$ \\
\hline 98 & Flutolanil & 18.30 & 323 & 0.003 & 81 & 9 & 0.025 & 86 & 8 & $\leq 0.001$ \\
\hline 99 & Fluvalinate, tau- & 24.80 & 250 & 0.025 & 95 & 11 & 0.245 & 95 & 5 & 0.004 \\
\hline 100 & Folpet & 17.65 & 147 & 0.016 & 96 & 16 & 0.159 & 91 & 15 & 0.009 \\
\hline 101 & Fonofos & 14.55 & 246 & 0.005 & 94 & 6 & 0.049 & 92 & 7 & 0.001 \\
\hline 102 & Formetanate & 15.27 & 122 & 0.05 & * & $*$ & 0.498 & 102 & $\underline{62}$ & 0.188 \\
\hline 103 & Formothion & 15.27 & 170 & 0.005 & 102 & 13 & 0.049 & 89 & $\overline{4}$ & 0.004 \\
\hline 104 & Fuberidazole & 15.79 & 184 & 0.005 & 83 & 29 & 0.051 & $\underline{55}$ & 17 & 0.001 \\
\hline 105 & Furalaxyl & 17.59 & 242 & 0.005 & 95 & 10 & 0.051 & $\overline{101}$ & 9 & 0.002 \\
\hline 106 & Heptachlor & 12.19 & 272 & 0.001 & $*$ & $*$ & 0.014 & 92 & 5 & 0.003 \\
\hline 107 & Heptachlorepoxide-I & 17.45 & 353 & 0.003 & $*$ & $*$ & 0.033 & 97 & 12 & 0.004 \\
\hline 108 & Heptachlorepoxide-II & 17.36 & 353 & 0.001 & 96 & 13 & 0.015 & 94 & 8 & $\leq 0.001$ \\
\hline 109 & Heptenophos & 12.24 & 124 & 0.003 & 95 & 5 & 0.03 & 93 & 3 & $\leq 0.001$ \\
\hline 110 & Hexachlorobenzene & 18.33 & 284 & 0.005 & 75 & 28 & 0.049 & 96 & 15 & 0.001 \\
\hline 111 & Hexaconazole & 18.32 & 216 & 0.002 & * & $*$ & 0.02 & 87 & 7 & 0.003 \\
\hline 112 & Imazalil & 18.37 & 215 & 0.005 & 79 & $\underline{50}$ & 0.05 & 77 & 14 & 0.002 \\
\hline
\end{tabular}


Table 5 (continued)

\begin{tabular}{|c|c|c|c|c|c|c|c|c|c|c|}
\hline & Pesticide & $t_{\mathrm{R}}(\min )$ & $m / z$ (quant) & Level $\left(\mathrm{mg} \mathrm{kg}^{-1}\right)$ & Rec. (\%) & RSD (\%) & Level $\left(\mathrm{mg} \mathrm{kg}^{-1}\right)$ & Rec. $(\%)$ & RSD (\%) & $\operatorname{LOD}\left(\mathrm{mg} \mathrm{kg}^{-1}\right)$ \\
\hline 113 & Iprodione & 20.75 & 316 & 0.012 & 108 & 7 & 0.12 & 95 & 4 & 0.004 \\
\hline 114 & Isofenphos & 17.46 & 213 & 0.005 & $*$ & $*$ & 0.051 & 93 & 3 & 0.010 \\
\hline 115 & Jasmolin-I & 19.36 & 123 & 0.053 & $*$ & $*$ & 0.528 & 77 & 5 & 0.100 \\
\hline 116 & Kresoxim-methyl & 18.73 & 206 & 0.014 & 95 & 6 & 0.139 & 91 & 9 & 0.005 \\
\hline 117 & Lindane & 14.41 & 183 & 0.002 & 86 & 18 & 0.02 & 99 & 6 & 0.001 \\
\hline 118 & Linuron & 16.35 & 248 & 0.005 & $*$ & $*$ & 0.048 & 79 & 9 & 0.010 \\
\hline 119 & Lufenuron (deg) & 11.48 & 176 & 0.011 & $\underline{123}$ & 20 & 0.114 & 76 & 34 & 0.004 \\
\hline 120 & Malathion & 16.43 & 173 & 0.003 & $*$ & $*$ & 0.034 & 98 & 5 & 0.005 \\
\hline 121 & Mecarbam & 17.49 & 329 & 0.003 & $*$ & $*$ & 0.029 & 93 & 5 & 0.004 \\
\hline 122 & Mepanipyrim & 18.07 & 222 & 0.001 & $*$ & $*$ & 0.013 & 92 & 8 & 0.002 \\
\hline 123 & Mepronil & 19.54 & 269 & 0.002 & $*$ & $*$ & 0.023 & 87 & 10 & 0.005 \\
\hline 124 & Metalaxyl & 15.95 & 206 & 0.003 & 92 & 10 & 0.028 & 97 & 5 & 0.002 \\
\hline 125 & Metaldehyde & 8.87 & 89 & 0.005 & $*$ & $*$ & 0.05 & $\underline{111}$ & $\underline{62}$ & 0.021 \\
\hline 126 & Methacrifos & 11.28 & 180 & 0.003 & 97 & 17 & 0.029 & $\overline{85}$ & $\overline{4}$ & $\leq 0.001$ \\
\hline 127 & Methamidophos & 7.75 & 141 & 0.026 & 36 & 24 & 0.258 & 47 & 15 & 0.005 \\
\hline 128 & Methidathion & 17.82 & 145 & 0.003 & 81 & $\overline{20}$ & 0.03 & 101 & 5 & 0.001 \\
\hline 129 & Methiocarb & 16.26 & 168 & 0.002 & 109 & $\underline{59}$ & 0.02 & 77 & $\underline{46}$ & 0.001 \\
\hline 130 & Methoxychlor & 21.03 & 228 & 0.002 & $*$ & * & 0.025 & 90 & $\overline{10}$ & 0.003 \\
\hline 131 & Metoprene & 17.56 & 73 & 0.01 & 104 & 5 & 0.103 & 93 & 3 & 0.003 \\
\hline 132 & Mevinphos & 10.36 & 192 & 0.003 & 104 & 16 & 0.03 & 99 & 1 & $\leq 0.001$ \\
\hline 133 & Monocrotophos & 13.43 & 192 & 0.046 & 84 & 8 & 0.456 & 88 & 7 & 0.021 \\
\hline 134 & Myclobutanil & 18.66 & 150 & 0.006 & $*$ & $*$ & 0.055 & 97 & 5 & 0.012 \\
\hline 135 & Nuarimol & 20.28 & 314 & 0.005 & $*$ & $*$ & 0.049 & 89 & 7 & 0.008 \\
\hline 136 & Omethoate & 12.39 & 156 & 0.005 & 57 & 19 & 0.054 & $\underline{53}$ & 14 & 0.002 \\
\hline 137 & Oxadixyl & 19.38 & 163 & 0.012 & * & $*$ & 0.124 & $\overline{92}$ & 4 & 0.038 \\
\hline 138 & Oxydemeton-methyl (deg) & 6.63 & 110 & 0.005 & $*$ & $*$ & 0.052 & 79 & 7 & 0.010 \\
\hline 139 & Paclobutrazole & 18.11 & 238 & 0.007 & 197 & 28 & 0.07 & 90 & 6 & $\leq 0.001$ \\
\hline 140 & Parathion & 16.69 & 291 & 0.011 & $\overline{106}$ & $\underline{26}$ & 0.106 & 91 & 6 & 0.004 \\
\hline 141 & Parathion-methyl & 15.71 & 263 & 0.002 & 88 & 7 & 0.021 & 94 & 2 & $\leq 0.001$ \\
\hline 142 & Penconazole & 17.35 & 248 & 0.003 & 90 & 10 & 0.03 & 94 & 4 & $\leq 0.001$ \\
\hline 143 & Permethrin-cis & 22.65 & 183 & 0.005 & 101 & 7 & 0.049 & 98 & 7 & 0.003 \\
\hline 144 & Permethrin-trans & 22.77 & 183 & 0.001 & $*$ & $*$ & 0.011 & 98 & 7 & 0.001 \\
\hline 145 & Phenothrin-I & 21.40 & 183 & 0.005 & 97 & 8 & 0.05 & 92 & 9 & 0.001 \\
\hline 146 & Phenothrin-II & 21.51 & 123 & 0.005 & 93 & 6 & 0.05 & 93 & 10 & 0.004 \\
\hline 147 & Phenthoate & 17.53 & 274 & 0.005 & 103 & 8 & 0.048 & 91 & 5 & 0.001 \\
\hline 148 & Phenylphenol, 2- & 11.56 & 170 & 0.005 & 96 & 6 & 0.052 & 95 & 4 & 0.001 \\
\hline 149 & Phorate & 13.56 & 260 & 0.005 & 98 & 6 & 0.05 & 92 & 5 & 0.001 \\
\hline 150 & Phosalone & 21.61 & 182 & 0.001 & $\underline{117}$ & 5 & 0.009 & 101 & 5 & $\leq 0.001$ \\
\hline 151 & Phosmet & 20.90 & 160 & 0.005 & $\underline{123}$ & 16 & 0.052 & 100 & 4 & $\leq 0.001$ \\
\hline 152 & Phosphamidon-I & 14.75 & 127 & 0.011 & $\overline{93}$ & 16 & 0.105 & 90 & 3 & 0.002 \\
\hline 153 & Phosphamidon-II & 15.49 & 127 & 0.011 & 89 & 9 & 0.105 & 91 & 2 & 0.005 \\
\hline 154 & Piperonyl butoxide & 20.36 & 176 & 0.004 & $*$ & $*$ & 0.037 & 89 & 10 & 0.010 \\
\hline 155 & Pirimicarb & 15.25 & 166 & 0.002 & 101 & 9 & 0.02 & 95 & 5 & $\leq 0.001$ \\
\hline 156 & Pirimiphos-methyl & 16.26 & 233 & 0.002 & $*$ & $*$ & 0.016 & 87 & 2 & 0.004 \\
\hline 157 & Prochloraz & 22.97 & 180 & 0.004 & * & $*$ & 0.038 & 101 & 6 & 0.007 \\
\hline 158 & Procymidone & 17.68 & 285 & 0.003 & 104 & 15 & 0.029 & 91 & 7 & 0.001 \\
\hline 159 & Profenofos & 18.42 & 337 & 0.005 & 97 & 8 & 0.052 & 95 & 10 & 0.001 \\
\hline 160 & Propargite & 20.31 & 350 & 0.01 & $*$ & $*$ & 0.102 & 96 & 7 & 0.020 \\
\hline 161 & Propham & 10.73 & 179 & 0.005 & 97 & 5 & 0.049 & 94 & 5 & 0.001 \\
\hline 162 & Propiconazole-I & 19.89 & 259 & 0.014 & 92 & 5 & 0.141 & 89 & 9 & 0.003 \\
\hline 163 & Propiconazole-II & 20.02 & 259 & 0.014 & 90 & 5 & 0.141 & 87 & 9 & 0.002 \\
\hline 164 & Propoxur & 12.62 & 110 & 0.002 & 96 & 6 & 0.02 & 92 & 7 & $\leq 0.001$ \\
\hline 165 & Propyzamide & 14.58 & 175 & 0.005 & 76 & $\underline{39}$ & 0.046 & 99 & 2 & 0.001 \\
\hline 166 & Prothiofos & 18.37 & 267 & 0.003 & 85 & $\overline{19}$ & 0.032 & 101 & 9 & 0.001 \\
\hline 167 & Pyrazophos & 22.17 & 221 & 0.003 & 137 & 11 & 0.03 & 145 & 4 & $\leq 0.001$ \\
\hline 168 & Pyrethrins & 19.62 & 123 & 0.053 & $*$ & $*$ & 0.528 & $\overline{99}$ & 13 & 0.087 \\
\hline
\end{tabular}


Table 5 (continued)

\begin{tabular}{|c|c|c|c|c|c|c|c|c|c|c|}
\hline & Pesticide & $t_{\mathrm{R}}(\min )$ & $m / z$ (quant) & Level $\left(\mathrm{mg} \mathrm{kg}^{-1}\right)$ & Rec. $(\%)$ & RSD (\%) & Level $\left(\mathrm{mg} \mathrm{kg}^{-1}\right)$ & Rec. (\%) & RSD (\%) & $\mathrm{LOD}\left(\mathrm{mg} \mathrm{kg}^{-1}\right)$ \\
\hline 169 & Pyridaben & 22.82 & 147 & 0.005 & 96 & 9 & 0.051 & 94 & 3 & 0.001 \\
\hline 170 & Pyridaphenthion & 20.80 & 199 & 0.005 & 99 & 10 & 0.048 & 93 & 5 & 0.003 \\
\hline 171 & Pyrifenox-I & 17.39 & 262 & 0.011 & 84 & 7 & 0.106 & 95 & 6 & 0.003 \\
\hline 172 & Pyrifenox-II & 14.68 & 264 & 0.011 & $*$ & $*$ & 0.106 & 90 & 6 & 0.170 \\
\hline 173 & Pyrimethanil & 14.65 & 198 & 0.002 & $\underline{135}$ & 14 & 0.02 & $\underline{123}$ & 4 & $\leq 0.001$ \\
\hline 174 & Pyriproxyfen & 21.65 & 136 & 0.002 & $\overline{119}$ & 18 & 0.024 & 91 & 6 & $\leq 0.001$ \\
\hline 175 & Quinalphos & 17.55 & 146 & 0.004 & $\overline{70}$ & 9 & 0.041 & 87 & 8 & 0.002 \\
\hline 176 & Quinoxyfen & 19.90 & 272 & 0.001 & 113 & 13 & 0.014 & 105 & 13 & $\leq 0.001$ \\
\hline 177 & Quintozene & 14.50 & 237 & 0.005 & $\overline{106}$ & 10 & 0.046 & 108 & 2 & 0.003 \\
\hline 178 & Simazine & 16.17 & 201 & 0.004 & 91 & 9 & 0.039 & 95 & 7 & 0.002 \\
\hline 179 & Spiroxamine & 15.67 & 198 & 0.018 & 99 & 17 & 0.176 & 81 & 2 & 0.009 \\
\hline 180 & TDE, o,p'- & 18.67 & 235 & 0.003 & 99 & 5 & 0.028 & 95 & 4 & $\leq 0.001$ \\
\hline 181 & TDE, $p, p^{\prime}-$ & 19.36 & 235 & 0.001 & 86 & 10 & 0.014 & 90 & 7 & $\leq 0.001$ \\
\hline 182 & Tebuconazole & 20.28 & 250 & 0.009 & $*$ & $*$ & 0.089 & 91 & 9 & 0.031 \\
\hline 183 & Tebufenpyrad & 21.12 & 171 & 0.005 & 92 & 17 & 0.052 & 87 & 7 & 0.001 \\
\hline 184 & Tecnazene & 12.56 & 203 & 0.005 & 108 & 6 & 0.048 & 99 & 6 & 0.002 \\
\hline 185 & Teflubenzuron (deg) & 8.12 & 197 & 0.003 & $\underline{174}$ & 25 & 0.025 & $\underline{124}$ & $\underline{25}$ & 0.002 \\
\hline 186 & Tefluthrin & 14.91 & 197 & 0.001 & * & $*$ & 0.014 & 89 & $\overline{14}$ & 0.002 \\
\hline 187 & Terbufos & 14.46 & 231 & 0.005 & 100 & 8 & 0.052 & 95 & 3 & $\leq 0.001$ \\
\hline 188 & Tetraconazole & 16.85 & 336 & 0.003 & 95 & 3 & 0.026 & 88 & 6 & $\leq 0.001$ \\
\hline 189 & Tetradifon & 21.44 & 356 & 0.003 & $*$ & $*$ & 0.03 & 94 & 8 & 0.010 \\
\hline 190 & Thiometon & 13.78 & 88 & 0.005 & 93 & 5 & 0.055 & 100 & 3 & $\leq 0.001$ \\
\hline 191 & Tolclofos-methyl & 15.80 & 265 & 0.001 & 91 & 6 & 0.01 & 102 & 5 & $\leq 0.001$ \\
\hline 192 & Tolylfluanid & 17.42 & 238 & 0.003 & 85 & 17 & 0.031 & 96 & 2 & 0.002 \\
\hline 193 & Triadimefon & 16.75 & 208 & 0.007 & 90 & 14 & 0.065 & 97 & 6 & 0.005 \\
\hline 194 & Triadimenol & 17.85 & 168 & 0.005 & $*$ & * & 0.053 & 85 & 2 & 0.029 \\
\hline 195 & Triazamate & 17.95 & 242 & 0.003 & $*$ & $*$ & 0.028 & 90 & 10 & 0.010 \\
\hline 196 & Triazophos & 19.62 & 257 & 0.005 & 109 & $\underline{37}$ & 0.054 & 89 & 20 & 0.001 \\
\hline 197 & Trifloxystrobin & 19.92 & 116 & 0.006 & 91 & $\overline{13}$ & 0.055 & 88 & 11 & 0.002 \\
\hline 198 & Triflumizole & 17.70 & 278 & 0.007 & 102 & 15 & 0.066 & 80 & 15 & 0.001 \\
\hline 199 & Trifluralin & 13.33 & 306 & 0.002 & 92 & 19 & 0.019 & 94 & 8 & $\leq 0.001$ \\
\hline 200 & Vamidothion & 17.95 & 87 & 0.019 & $*$ & $*$ & 0.187 & 100 & 5 & 0.045 \\
\hline 201 & Vinclozolin & 15.71 & 198 & 0.005 & 97 & 16 & 0.047 & 93 & 7 & 0.003 \\
\hline
\end{tabular}

${ }^{\text {a }}$ Benzoylurea(deg) $=2,4$-difluorobenzamide

LOD: Amount for which $S / N=3$, or in the event of an interfering peak, the average peak height for fortified sample $(n=5)$ should be 3.3 times the average peak height for control sample $(n=2)$

*Fortification level below LOD as defined above

Underlined values are outside EU criteria for method validation

recovery, and selectivity with other matrices. For this, with each analytical batch, one of the samples submitted for routine analysis was spiked with 135 pesticides at five times the target LOQ level (i.e. samples were spiked with $0.05 \mathrm{mg} \mathrm{kg}^{-1}$ of most of the pesticides). A compilation was made of recovery data from a period of 15 months which included analysis of approximately 100 different vegetable and fruit commodities. Given the wide variety of commodities, matrix-matched calibration is quite tedious and would substantially increase the number of standard solutions to be analyzed in the GC sequence. It was therefore decided to select one relatively simple matrix (tomato) as default for matrix-matched calibration, i.e. recoveries for all commodities were calculated against the tomato-matrix standard.
For each pesticide, calculations were performed for two diagnostic ions. All together this resulted in approximately 30,000 values.

According to the current EU guideline on quality control in pesticide residue analysis [37], the recovery obtained during routine analysis should be within $60-140 \%$. An overview of the percentage of recovery values within or outside the 60 $140 \%$ criterion for a wide variety of matrices is presented in Table 6. With such large number of pesticides (or, actually, diagnostic ions) and matrices, one failing combination or more occurred for most matrices. There are several causes for this. Main reasons for recovery below $60 \%$ could be poor extraction efficiency or incomplete transfer of the pesticides to the GC column (e.g. adsorption and/or degradation in a 
contaminated inlet). Higher recovery may occur when a compound from the matrix generates the same diagnostic ion as a pesticide and co-elutes with that pesticide (i.e. detection was not selective). Another reason could be that the matrix effect induced in the GC inlet [50] for a pesticide in a particular matrix is more pronounced than that in the tomatobased calibration standard.

Failing pesticide-matrix combinations were most abundant for herbs, kale, sweetcorn, and golden berry, for which up to $35 \%$ of recovery values (calculated using the two diagnostic ions for each pesticide) were outside the 60 $140 \%$ range. These products contain larger amounts of coextractants than most other vegetables and fruits, which may result in insufficient detection selectivity, enhanced response as a result of a matrix effect (more shielding of active sites in the inlet), and contamination of the inlet. For this type of product more selectivity, e.g. by use of MS-MS would be beneficial. Such detection is also more sensitive than single quadrupole full-scan detection and would enable reduction in the amount of matrix introduced, thus reducing build up of contamination. Overall, when data for all 110 QC samples were included, recovery was acceptable for $91 \%$ of the diagnostic ions measured.

On the basis of the same data, an overview by pesticide is presented in Table 7. For each pesticide two diagnostic ions from the full-scan data were integrated and concentrations were calculated. In routine practice, however, the most convenient way of reviewing the data is by using one and the same diagnostic ion for each pesticide, irrespective the matrix. On the basis of the data set obtained (nearly 14,700 pesticide-matrix combinations) the most favorable of the two diagnostic ions, i.e. the ion for which the highest number of recoveries within $60-140 \%$ was obtained, was assigned as the Quan ion (default quantification ion). By using this ion, acceptable recoveries were obtained for $93 \%$ of pesticides-matrix combinations. This also means that $7 \%$ or, in absolute figures, 1008 of the pesticide-matrix combinations did not meet the criterion. $40 \%$ of these failing combinations could be accepted after use of the alternative ion, for which calculations were also performed automatically during data processing. Low recoveries $(<60 \%)$ for both diagnostic ions were obtained for $2.7 \%$ of pesticidematrix combinations. High recoveries $(>140 \%)$ were obtained for $2 \%$ of the combinations. For this latter group manual evaluation of other ions, if available and sufficiently abundant, could further increase the number of acceptable recoveries. Because this is a time-consuming process, it was not done routinely. In the event of deviating recovery, assessment of the results to be reported was based on visual evaluation of the extracted ion chromatograms of the two diagnostic ions at least. On the basis of on the findings it was then concluded the pesticide could not
Fig. 4 GC-MS extracted-ion chromatograms obtained from lettuce with (upper traces) and without fortification with pesticides, and the corresponding mass spectra (upper, reference spectra; lower, background-corrected spectra from the sample). a, b, $0.005 \mathrm{mg} \mathrm{kg}^{-1}$ disulfoton $(\mathrm{m} / \mathrm{z} 88)$; c, d, $0.002 \mathrm{mg} \mathrm{kg}^{-1}$ fipronil $(\mathrm{m} / \mathrm{z} 367)$; e, f, $0.006 \mathrm{mg} \mathrm{kg}^{-1}$ biphenyl $(\mathrm{m} / \mathrm{z} 154)$

be determined in that specific matrix, or only at higher levels.

It should be noted that the above evaluation applies to a level five times the reporting level, which was set at $0.01 \mathrm{mg} \mathrm{kg}^{-1}$, or the LOQ if higher than $0.01 \mathrm{mg} \mathrm{kg}^{-1}$. At lower levels interferences may have a larger effect and, consequently, more frequent deviations from the $60-140 \%$ criterion (most probably $>140 \%$ ) may be observed. For higher levels, the opposite would be true.

Pesticides for which low recoveries $(<60 \%)$ were frequently obtained (10-21 of 110 QC samples) included iprodione and $p, p^{\prime}$-DDT (degradation in inlet), dimethomorph (polar, relatively non-volatile, could be troublesome in splitless transfer), pentachloroanisole, pentachloroaniline, and mepanipyrim (no clear explanation, but probably related to the dispersive SPE clean-up). There were no indications for poor extraction efficiency.

High recovery $(>140 \%)$ frequently occurred for etridiazole, methidathion, mevinphos, phosmet, phosalone, phosphamidone, and endosulfan-alpha (10-21 times out of 110 QC samples, often in herbs and peas). This was attributed to matrix effects and interferences.

Overall, the pesticides that failed most frequently (11-28 times out of 110) during routine analytical quality control were (in descending order) etridiazole, iprodione, methidathion, pentachlorothioanisole, mevinphos, phosmet, $p, p^{\prime}$ DDT, mepanipyrim, phosalone, phosphamidon, biphenyl, dichlorvos, spirodiclofen, pentachloroaniline, deltamethrin, tau-fluvalinate, and pyrazophos. These would be the most relevant for inclusion in alternative methods, for example GC-MS-MS or LC-MS-MS.

Average recovery and RSD were calculated for pesticidematrix combinations that passed the acceptable recovery criterion. The results are included in Table 7. Average recovery was usually close to $100 \%$ and RSDs approximately $15 \%$. For the pesticides known to be adsorbed by GCB systematically lower average recovery $(77-90 \%)$ was obtained, which is in agreement with the results obtained during method development.

These comprehensive data show that with a relatively inexpensive single-quadrupole MS detector in full-scan mode it is possible to obtain reliable quantitative data down to the $0.01 \mathrm{mg} \mathrm{kg}^{-1}$ level, or even lower, for a wide range of pesticides in a wide variety of matrices after generic rapid sample preparation based on extraction with ethyl acetate. Unified calibration based on a tomato-matrix standard is, 
$$
{ }^{100}
$$

a

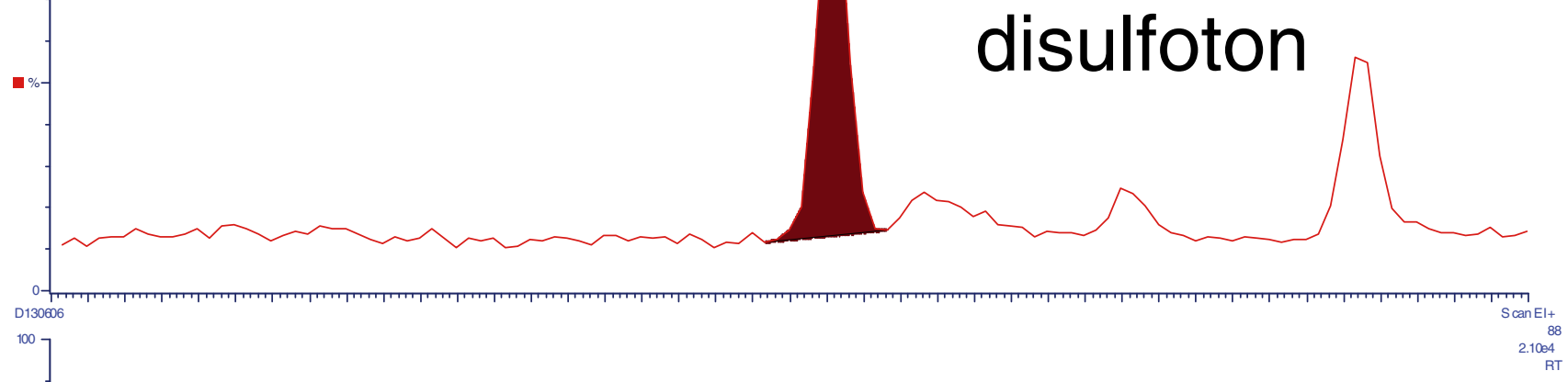

$-$
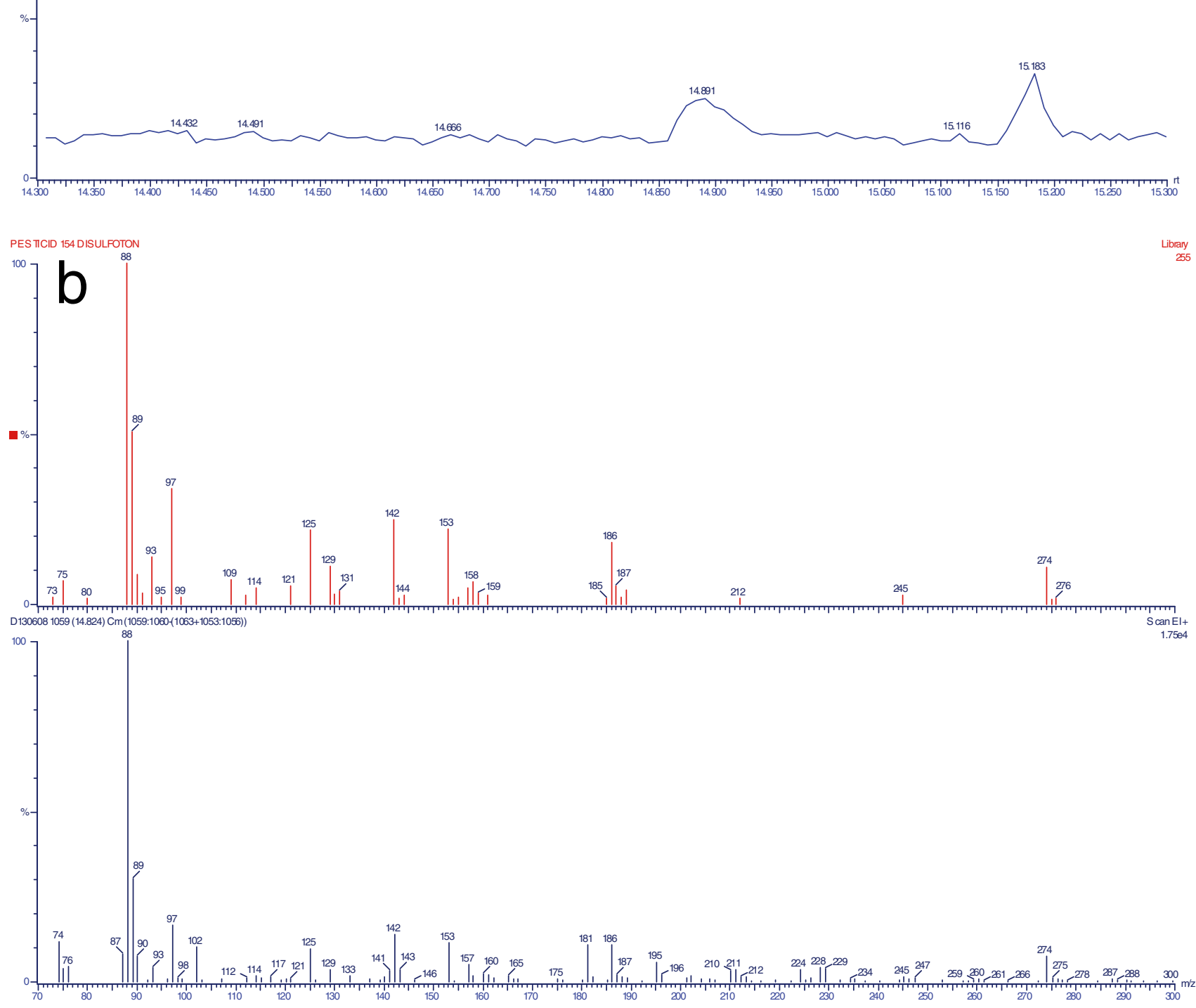
Sample ID: 04 rec 1// 0 LOQ 02

Acquired on 13-Jun-2002 at 23:27:5

D130008



PESTICID 294 FIPRONIL

7
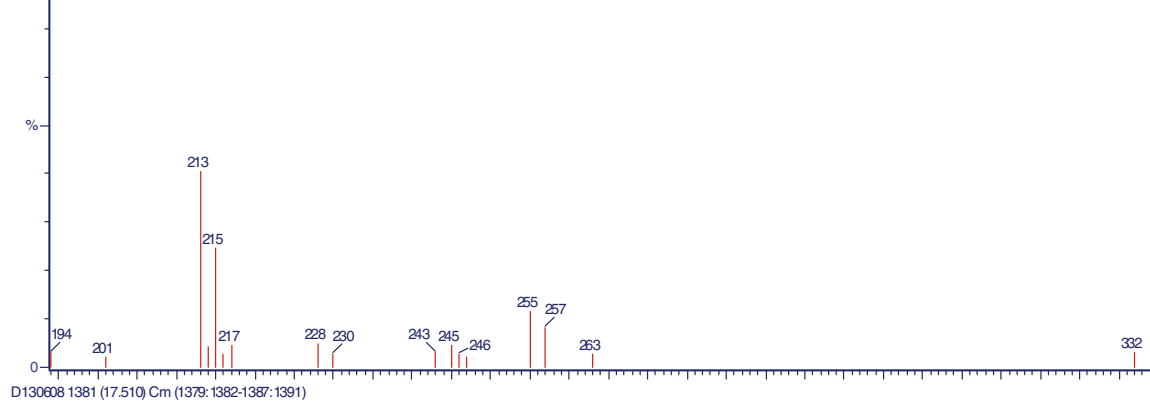

1007205

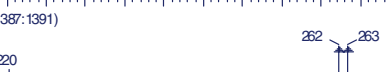

\begin{tabular}{l|l|l|}
208 & \\
196 & 200
\end{tabular}

$\left.\left.\right|_{\mid} ^{196}\right|^{208}$

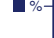

$\left.\right|^{197}||^{210} \quad 2$

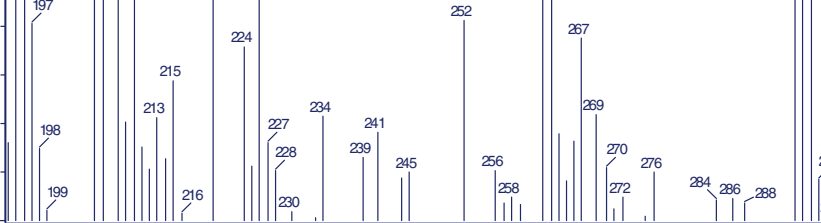

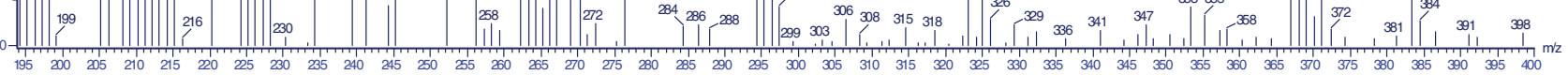

fig. 4 (continued) 
$\left.{ }^{100}\right] \mathbf{e}$

e
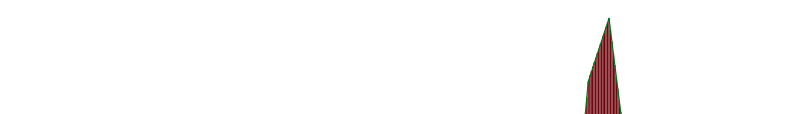

\section{$0.006 \mathrm{mg} / \mathrm{kg}$ \\ biphenyl}

$\square$
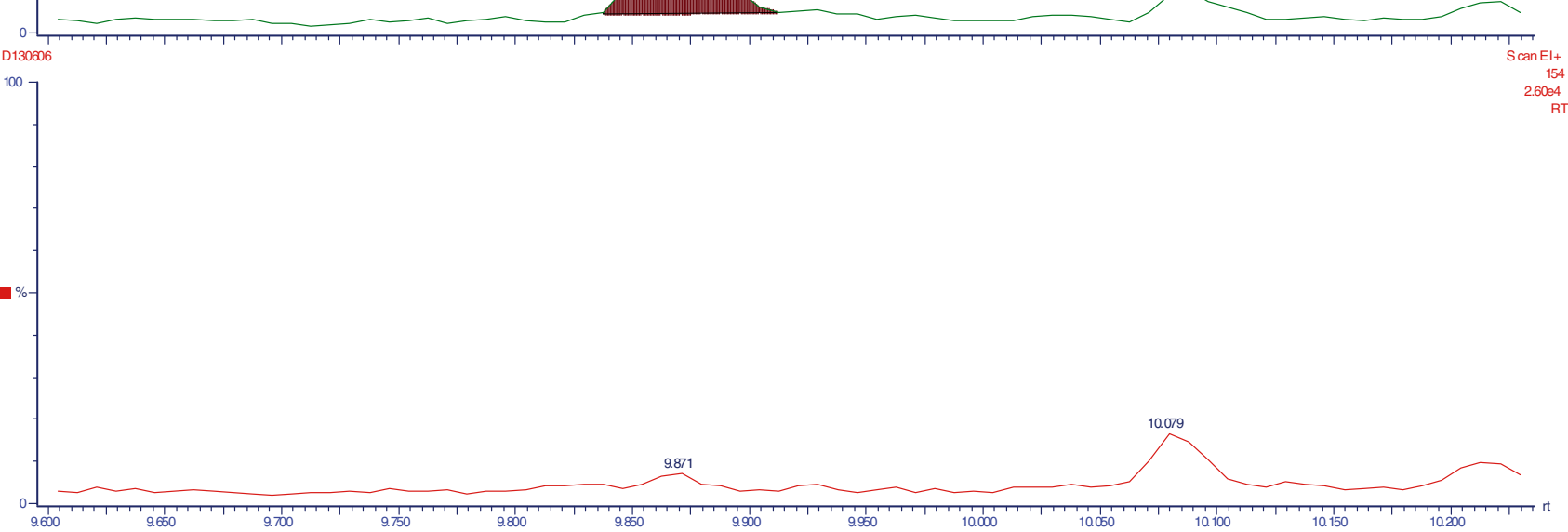

PESTICID 254 BIPHENYL


fig. 4 (continued) 
Table 6 Overview of percentage of recovery values ${ }^{\mathrm{a}}$ within or outside the EU $60-140 \%$ criterion [37] after GC-MS analysis

\begin{tabular}{|c|c|c|c|c|}
\hline & \multirow[t]{2}{*}{ Matrix } & \multicolumn{3}{|c|}{ Percentage of all recovery values ${ }^{\mathrm{a}}$} \\
\hline & & $60-140 \%$ & $<60 \%$ & $>140 \%$ \\
\hline 1 & Beetroot & 100 & 0 & 0 \\
\hline 2 & Cucumber (1/2) & 100 & 0 & 0 \\
\hline 3 & $\operatorname{Mint}(1 / 2)$ & 100 & 0 & 0 \\
\hline 4 & Sharonfruit $(1 / 2)$ & 100 & 0 & 0 \\
\hline 5 & Witloof & 100 & 0 & 0 \\
\hline 6 & Asparagus & 99 & 1 & 0 \\
\hline 7 & Bean sprouts & 99 & 0 & 1 \\
\hline 8 & Corn syrup & 99 & 0 & 0 \\
\hline 9 & Fennel leaves & 99 & 0 & 1 \\
\hline 10 & Grape & 99 & 0 & 1 \\
\hline 11 & Kohlrabi (1/3) & 99 & 1 & 0 \\
\hline 12 & Lima bean & 99 & 0 & 1 \\
\hline 13 & Pak choi (1/2) & 99 & 0 & 1 \\
\hline 14 & Pear concentrate & 99 & 0 & 1 \\
\hline 15 & Pumpkin & 99 & 0 & 1 \\
\hline 16 & Salsify & 99 & 0 & 0 \\
\hline 17 & Sharonfruit $(2 / 2)$ & 99 & 0 & 1 \\
\hline 18 & Strawberry & 99 & 0 & 1 \\
\hline 19 & Sugar pea & 99 & 1 & 0 \\
\hline 20 & Taro & 99 & 0 & 1 \\
\hline 21 & Bitter cucumber & 98 & 0 & 2 \\
\hline 22 & Cucumber $(2 / 2)$ & 98 & 1 & 1 \\
\hline 23 & Egg plant & 98 & 0 & 2 \\
\hline 24 & Kidney bean & 98 & 1 & 1 \\
\hline 25 & Kohlrabi $(2 / 3)$ & 98 & 1 & 1 \\
\hline 26 & Mushroom & 98 & 0 & 2 \\
\hline 27 & Pineapple & 98 & 1 & 1 \\
\hline 28 & Sweet pepper & 98 & 0 & 2 \\
\hline 29 & Tomato puree (processed) & 98 & 0 & 2 \\
\hline 30 & Turnip & 98 & 1 & 0 \\
\hline 31 & Turnip tops $(1 / 2)$ & 98 & 0 & 2 \\
\hline 32 & Alfalfa & 97 & 1 & 2 \\
\hline 33 & Cauliflower & 97 & 1 & 2 \\
\hline 34 & Cherry & 97 & 0 & 3 \\
\hline 35 & Chestnut & 97 & 2 & 1 \\
\hline 36 & Endive & 97 & 0 & 3 \\
\hline 37 & Fig & 97 & 0 & 3 \\
\hline 38 & Kangkung (1/2) & 97 & 1 & 2 \\
\hline 39 & Kangkung (2/2) & 97 & 2 & 1 \\
\hline 40 & Ladies' fingers & 97 & 0 & 3 \\
\hline 41 & Mango & 97 & 0 & 3 \\
\hline 42 & Pear puree (processed) & 97 & 0 & 3 \\
\hline 43 & Sorrel & 97 & 3 & 0 \\
\hline 44 & Soybean sprouts & 97 & 0 & 3 \\
\hline 45 & Asparagus bean & 96 & 1 & 3 \\
\hline 46 & Orange & 96 & 2 & 2 \\
\hline 47 & Potato leaves & 96 & 2 & 2 \\
\hline 48 & Rhubarb & 96 & 2 & 2 \\
\hline 49 & Artichoke & 95 & 0 & 5 \\
\hline 50 & Tangelo & 95 & 2 & 3 \\
\hline 51 & Tarrragon & 95 & 3 & 2 \\
\hline 52 & Wine (red) & 95 & 1 & 4 \\
\hline 53 & Apricot & 94 & 0 & 6 \\
\hline 54 & Chives $(1 / 3)$ & 94 & 3 & 3 \\
\hline 55 & Chives $(2 / 3)$ & 94 & 4 & 2 \\
\hline 56 & Dill leaves & 94 & 4 & 2 \\
\hline 57 & Melon puree (processed) & 94 & 1 & 5 \\
\hline
\end{tabular}

Table 6 (continued)

\begin{tabular}{|c|c|c|c|c|}
\hline & \multirow[t]{2}{*}{ Matrix } & \multicolumn{3}{|c|}{ Percentage of all recovery values ${ }^{\mathrm{a}}$} \\
\hline & & $60-140 \%$ & $<60 \%$ & $>140 \%$ \\
\hline 58 & Mineola & 94 & 1 & 6 \\
\hline 59 & Pak choi $(2 / 2)$ & 94 & 2 & 4 \\
\hline 60 & Sugar water & 94 & 6 & 0 \\
\hline 61 & Broad bean & 93 & 1 & 6 \\
\hline 62 & Celery leaves $(1 / 4)$ & 93 & 3 & 4 \\
\hline 63 & Chervil & 93 & 5 & 2 \\
\hline 64 & Dates & 93 & 7 & 0 \\
\hline 65 & Sweetcorn $(1 / 3)$ & 93 & 4 & 3 \\
\hline 66 & Carrot & 92 & 1 & 7 \\
\hline 67 & Haricot bean & 92 & 0 & 8 \\
\hline 68 & Oregano & 92 & 5 & 3 \\
\hline 69 & Parsnip & 92 & 2 & 6 \\
\hline 70 & Fennel & 91 & 0 & 9 \\
\hline 71 & Green pea $(1 / 2)$ & 91 & 4 & 5 \\
\hline 72 & Passion fruit (1/2) & 91 & 2 & 7 \\
\hline 73 & Celery leaves $(2 / 4)$ & 90 & 6 & 4 \\
\hline 74 & Green pea $(2 / 2)$ & 90 & 1 & 9 \\
\hline 75 & Lemon puree & 90 & 8 & 2 \\
\hline 76 & Mint $(2 / 2)$ & 90 & 5 & 5 \\
\hline 77 & Pomegranate & 90 & 1 & 9 \\
\hline 78 & Purslane & 90 & 1 & 9 \\
\hline 79 & Water cress & 90 & 2 & 8 \\
\hline 80 & Lettuce & 89 & 7 & 4 \\
\hline 81 & Chili pepper $(1 / 2)$ & 88 & 6 & 6 \\
\hline 82 & Chinese cabbage & 87 & 0 & 13 \\
\hline 83 & Passion fruit $(2 / 2)$ & 87 & 3 & 10 \\
\hline 84 & Bamboo shoots & 86 & 0 & 14 \\
\hline 85 & Celery leaves (3/4) & 86 & 7 & 7 \\
\hline 86 & Honey & 86 & 14 & 0 \\
\hline 87 & Potato puree (processed) & 86 & 14 & 0 \\
\hline 88 & Sugar pea & 85 & 0 & 15 \\
\hline 89 & Turnip tops $(2 / 2)$ & 85 & 0 & 15 \\
\hline 90 & Lime & 84 & 4 & 12 \\
\hline 91 & Blueberry & 83 & 2 & 16 \\
\hline 92 & Potato & 83 & 15 & 2 \\
\hline 93 & Celery leaves $(4 / 4)$ & 82 & 3 & 15 \\
\hline 94 & Green pea & 82 & 1 & 17 \\
\hline 95 & Apple pulp (processed) & 81 & 6 & 13 \\
\hline 96 & Cassava & 81 & 9 & 10 \\
\hline 97 & Chives $(3 / 3)$ & 81 & 7 & 12 \\
\hline 98 & Kohlrabi (3/3) & 78 & 0 & 22 \\
\hline 99 & Parsley (1/2) & 78 & 6 & 16 \\
\hline 100 & Thyme (1/3) & 78 & 2 & 20 \\
\hline 101 & Kale & 77 & 6 & 17 \\
\hline 102 & Chili pepper $(2 / 2)$ & 76 & 15 & 9 \\
\hline 103 & Coriander leaves & 76 & 18 & 6 \\
\hline 104 & Sweetcorn $(2 / 3)$ & 75 & 18 & 7 \\
\hline 105 & Sweetcorn $(3 / 3)$ & 74 & 9 & 17 \\
\hline 106 & Parsley $(2 / 2)$ & 73 & 20 & 7 \\
\hline 107 & Thyme $(2 / 3)$ & 73 & 3 & 24 \\
\hline 108 & Rocket & 72 & 3 & 25 \\
\hline 109 & Thyme (3/3) & 66 & 29 & 5 \\
\hline 110 & Golden berry (physalis) & 65 & 1 & 34 \\
\hline
\end{tabular}

a Recoveries at $0.05 \mathrm{mg} \mathrm{kg}^{-1}\left(0.10-0.30 \mathrm{mg} \mathrm{kg}^{-1}\right.$ for 22 pesticides $)$. Calculated for 135 pesticides, two diagnostic ions each, against a standard prepared in blank tomato extract. The pesticides included are listed in Table 7 
furthermore, a feasible approach. One should, however, be aware there are also limitations and that some pesticidematrix combinations cannot be determined in the $0.01-$ $0.1 \mathrm{mg} \mathrm{kg}^{-1}$ range, and that for other pesticides calibration against the corresponding matrix instead of tomato is required to bring quantitative results within the AQC criteria, especially for MRL violations, when more stringent criteria apply. The data also reveal that the only way to gain full insight into analyte recovery and method selectivity with a wide variety of matrices is by performing analytical quality control on all pesticides which are reported, rather than on a subset, as is suggested in the EU guideline [37]. A subset will suffice for demonstration of adequate sample preparation and injection but will not reveal limitations in the selectivity of GC-MS.

GC single-quadrupole MS remains an effective tool for routine $\mathrm{GC}$ analysis of pesticide residues. For many vegetable and fruit matrices there is no real need to change to more advanced (and expensive) MS techniques, for example MS-MS (which has limited scope) or accurate mass TOF-MS (which has a limited dynamic range). Use of such equipment would be justified for more complex matrices and when low $\mu \mathrm{g} \mathrm{kg}^{-1}$ LOQs are required-for example analysis of some pesticides in baby food.

\section{LC-MS-MS analysis}

\section{Clean-up}

The ethyl acetate extraction procedure is also appropriate for many pesticides not amenable to GC analysis $[11,15,16,18$, 26]. Typically no clean-up is performed (Table 1). One reason for this is that with regard to chromatographic performance LC columns tend to be more tolerant of injection of bulk matrix than GC columns. In our experience, continual injection of $20 \mathrm{mg}$ equivalent of vegetable and fruit extracts does not result in deterioration of chromatographic performance or unacceptable contamination of the ion source (the system used here was an API2000). In LC-MS co-extracted matrix does have an effect on the response, however, by interfering with the ionization process. This results in suppression (sometimes enhancement) of the response to a pesticide in a matrix compared with that in a solvent standard [51] and complicates quantification of pesticides in the samples. The possibility of reducing matrix effects by use of dispersive SPE clean-up was investigated in a similar way as for GC. First, the effectiveness of the clean-up step was investigated by addition of $25 \mathrm{mg}$ GCB and $25 \mathrm{mg}$ PSA to $1 \mathrm{~mL}$ raw extract of a mixed spinach-grape-onion sample $\left(1: 1: 1,1 \mathrm{~g} \mathrm{ml}^{-1}\right)$. Seventy pesticides (the ones in Table 8 with API2000 in the MS-MS column) were added after clean-up and analyzed by LC-MS-MS. The response was compared with that of solutions of equal concentration in the raw extract and a solvent standard. Clean-up increased the number of pesticides for which no pronounced matrix effect (less than $20 \%$ suppression or enhancement) was observed from 38 to $84 \%$. Several of the pesticides (Tables 2 and 4 ) were adsorbed by the SPE material, however. Although adsorption by the GCB could have been avoided or reduced by addition of toluene (although less practical when changing from extraction solvent to methanol/water), it was concluded that PSA was not compatible with a generic method for pesticides amenable to LC-MS-MS. It was therefore decided not to include a clean-up step for LC-MS-MS analysis and to use the initial raw ethyl acetate extract. Another reason for not further pursuing clean-up in LC-MS-MS analysis was that the sensitivity of current triple-quadrupole instruments enables injection of only small amounts of matrix into the LCMS-MS system (e.g. $2 \mathrm{mg}$ ) while still achieving the desired limits of quantification. Experiments showed that tenfold dilution of $1 \mathrm{~g} \mathrm{~mL}^{-1}$ extracts increased the number of pesticides for which no pronounced matrix effect occurred from 65 to $82 \%$ and from 10 to $65 \%$ for cucumber and cabbage, respectively.

Routine experience with LC-MS-MS analysis for over four years, both with the API2000 (20 mg matrix) and the API3000 (2 mg matrix) has shown that injection of uncleaned extracts does not result in special maintenance requirements. The source is cleaned with a tissue daily. The LC column typically lasts for 6 months.

\section{Changing the solvent}

Because ethyl acetate is less suitable for direct injection in reversed phase LC, the solvent was changed. Because only small amounts of the raw extract need to be evaporated (less than $0.5 \mathrm{~mL}$ in the final method) and evaporation blocks enable simultaneous evaporation of many (typically 24-36) extracts, this step adds little to the overall sample-preparation time. Changing the solvent was even regarded as advantageous. It resulted in more freedom in selection of the final solvent to be injected into the LC, which can be critical for very polar compounds (e.g. in acetonitrile-based extraction methods, injection of $100 \%$ acetonitrile easily leads to bandbroadening for methamidophos). It is also easier to compensate for the smaller amount of sample processed for dry crops (because of the need for addition of water) by evaporating a larger amount of the ethyl acetate extract.

In previous work [15] a small amount of a diethylene glycol (added as solution in methanol) was added, because this was found to facilitate reconstitution, thereby improving recovery for some pesticide--matrix combinations. It was also shown that the evaporation step did not require special attention and that continuing the process for another half hour after completion of evaporation of the solvent did not affect recovery. The same procedure was therefore used here 
Table 7 Recoveries over all matrices (GC-MS analysis)

Pesticide Quan. Qual. Fortification \# QCs matrices Both diagn. One of diagn. Both diagn. Both diagn. Average RSD ion $\mathrm{m} / \mathrm{z}$ ion $\mathrm{m} / \mathrm{z}$ level $\quad$ (see Table 6) ions $60-140 \%$ ions $60-140 \%$ ions $>140 \%$ ions $<60 \%$ recov. (\%) $\quad(\%)$ $\left(\mathrm{mg} \mathrm{kg}^{-1}\right)$

\begin{tabular}{|c|c|c|c|c|c|c|c|c|c|c|}
\hline Acrinathrin & 208 & 289 & 0.10 & 110 & 107 & 107 & 3 & 0 & 97 & 16 \\
\hline Azaconazole & 173 & 217 & 0.05 & 110 & 107 & 107 & 2 & 1 & 97 & 14 \\
\hline Azoxystrobin & 388 & 344 & 0.05 & 108 & 97 & 102 & 0 & 8 & 96 & 15 \\
\hline Benalaxyl & 206 & 148 & 0.05 & 110 & 108 & 109 & 0 & 1 & 100 & 13 \\
\hline Bifenthrin & 181 & 166 & 0.05 & 109 & 109 & 110 & 0 & 0 & 102 & 13 \\
\hline Biphenyl & 154 & 153 & 0.05 & 110 & 93 & 94 & 7 & 9 & 98 & 20 \\
\hline Boscalid & 112 & 140 & 0.13 & 109 & 98 & 100 & 2 & 8 & 96 & 16 \\
\hline Bromopropylate & 341 & 343 & 0.05 & 110 & 100 & 101 & 9 & 0 & 109 & 14 \\
\hline Bromuconazole & 295 & 173 & 0.05 & 110 & 100 & 105 & 4 & 1 & 102 & 18 \\
\hline Bupirimate & 273 & 208 & 0.02 & 110 & 108 & 109 & 0 & 1 & 96 & 15 \\
\hline Buprofezin & 172 & 105 & 0.05 & 109 & 105 & 108 & 2 & 0 & 102 & 12 \\
\hline Cadusafos & 158 & 159 & 0.05 & 110 & 105 & 107 & 1 & 2 & 104 & 13 \\
\hline Chlorfenapyr & 364 & 328 & 0.04 & 110 & 103 & 106 & 2 & 2 & 102 & 16 \\
\hline Chlorfenvinphos & 323 & 267 & 0.05 & 110 & 103 & 103 & 7 & 0 & 103 & 16 \\
\hline Chlorpropham & 213 & 127 & 0.05 & 108 & 101 & 106 & 2 & 2 & 105 & 14 \\
\hline Chlorpyrifos & 314 & 286 & 0.05 & 109 & 107 & 109 & 0 & 1 & 101 & 14 \\
\hline Chlorpyrifos-methyl & 288 & 286 & 0.05 & 108 & 101 & 104 & 4 & 2 & 102 & 16 \\
\hline Chlorthal-dimethyl & 332 & 301 & 0.05 & 110 & 110 & 110 & 0 & 0 & 101 & 14 \\
\hline Cinerin-1 & 123 & 150 & 0.11 & 110 & 104 & 105 & 4 & 1 & 101 & 15 \\
\hline Cyfluthrin & 226 & 199 & 0.20 & 110 & 102 & 106 & 0 & 4 & 100 & 17 \\
\hline Cyhalothrin, lambda- & 208 & 181 & 0.05 & 108 & 104 & 109 & 1 & 0 & 99 & 16 \\
\hline Cypermethrin & 163 & 181 & 0.15 & 105 & 99 & 107 & 2 & 0 & 102 & 14 \\
\hline Cyproconazole & 222 & 224 & 0.05 & 110 & 103 & 105 & 1 & 4 & 102 & 16 \\
\hline Cyprodinil & 224 & 225 & 0.05 & 109 & 101 & 102 & 0 & 8 & 85 & 15 \\
\hline $\mathrm{DDE}, p, p^{\prime}-$ & 246 & 318 & 0.06 & 110 & 110 & 110 & 0 & 0 & 101 & 13 \\
\hline DDT, $o, p^{\prime}-$ & 235 & 237 & 0.05 & 110 & 106 & 107 & 2 & 1 & 103 & 14 \\
\hline DDT, $p, p^{\prime}-$ & 237 & 235 & 0.05 & 110 & 82 & 90 & 9 & 11 & 98 & 20 \\
\hline Deltamethrin & 253 & 255 & 0.10 & 110 & 91 & 98 & 4 & 8 & 95 & 17 \\
\hline Diazinon & 179 & 137 & 0.05 & 109 & 108 & 110 & 0 & 0 & 101 & 13 \\
\hline Dichlorvos & 185 & 109 & 0.05 & 110 & 90 & 96 & 8 & 6 & 99 & 20 \\
\hline Dicloran & 206 & 160 & 0.05 & 108 & 96 & 102 & 3 & 5 & 99 & 15 \\
\hline Dieldrin & 263 & 79 & 0.05 & 110 & 109 & 109 & 0 & 1 & 104 & 14 \\
\hline Diethofencarb & 168 & 267 & 0.05 & 110 & 107 & 108 & 1 & 1 & 100 & 15 \\
\hline Difenoconazole & 323 & 265 & 0.10 & 107 & 101 & 106 & 0 & 4 & 96 & 16 \\
\hline Dimethipin & 118 & 76 & 0.05 & 110 & 95 & 104 & 5 & 1 & 104 & 16 \\
\hline Dimethomorph & 387 & 301 & 0.10 & 110 & 98 & 100 & 0 & 10 & 89 & 16 \\
\hline Dimoxystrobin & 205 & 116 & 0.05 & 110 & 108 & 109 & 0 & 1 & 100 & 12 \\
\hline Diniconazole & 270 & 268 & 0.15 & 64 & 58 & 62 & 1 & 1 & 97 & 17 \\
\hline Diphenylamine & 169 & 167 & 0.05 & 110 & 107 & 107 & 0 & 3 & 101 & 16 \\
\hline Dodemorph & 238 & 154 & 0.05 & 110 & 109 & 109 & 0 & 1 & 96 & 15 \\
\hline Endosulfan-alpha & $195+241$ & $239+197$ & 0.50 & 110 & 95 & 100 & 10 & 0 & 107 & 12 \\
\hline Endosulfan-beta & $195+241$ & $237+160$ & 0.10 & 110 & 107 & 107 & 3 & 0 & 102 & 14 \\
\hline Endosulfan-sulfate & $272+229$ & $274+237$ & 0.05 & 109 & 102 & 107 & 2 & 1 & 104 & 16 \\
\hline EPN & 157 & 323 & 0.05 & 110 & 103 & 106 & 3 & 1 & 103 & 17 \\
\hline Epoxiconazole & 192 & 138 & 0.05 & 110 & 106 & 108 & 1 & 1 & 98 & 14 \\
\hline Esfenvalerate & 167 & 125 & 0.15 & 110 & 102 & 103 & 4 & 3 & 106 & 15 \\
\hline Ethion & 231 & 153 & 0.05 & 110 & 106 & 106 & 4 & 0 & 103 & 14 \\
\hline Ethoprophos & 158 & 200 & 0.05 & 110 & 107 & 108 & 1 & 1 & 104 & 13 \\
\hline Etofenprox & 376 & 164 & 0.05 & 110 & 102 & 104 & 2 & 4 & 97 & 15 \\
\hline Etridiazole & 211 & 183 & 0.05 & 109 & 80 & 82 & 21 & 7 & 97 & 21 \\
\hline Fenarimol & 219 & 139 & 0.05 & 110 & 106 & 108 & 1 & 1 & 103 & 16 \\
\hline Fenazaquin & 160 & 145 & 0.05 & 110 & 105 & 105 & 1 & 4 & 88 & 16 \\
\hline Fenbuconazole & 129 & 198 & 0.05 & 110 & 105 & 107 & 1 & 2 & 99 & 17 \\
\hline Fenitrothion & 277 & 260 & 0.05 & 108 & 99 & 102 & 7 & 1 & 106 & 16 \\
\hline
\end{tabular}


Table 7 (continued)

\begin{tabular}{|c|c|c|c|c|c|c|c|c|c|c|}
\hline Pesticide & $\begin{array}{l}\text { Quan. } \\
\text { ion } m / z\end{array}$ & $\begin{array}{l}\text { Qual. } \\
\text { ion } m / z\end{array}$ & $\begin{array}{l}\text { Fortification } \\
\text { level } \\
\left(\mathrm{mg} \mathrm{kg}^{-1}\right)\end{array}$ & $\begin{array}{l}\text { \# QCs matrices } \\
\text { (see Table 6) }\end{array}$ & $\begin{array}{l}\text { Both diagn. } \\
\text { ions } 60-140 \%\end{array}$ & $\begin{array}{l}\text { One of diagn. } \\
\text { ions } 60-140 \%\end{array}$ & $\begin{array}{l}\text { Both diagn. } \\
\text { ions }>140 \%\end{array}$ & $\begin{array}{l}\text { Both diagn. } \\
\text { ions }<60 \%\end{array}$ & $\begin{array}{l}\text { Average } \\
\text { recov. (\%) } \\
\text { Quan. ion }\end{array}$ & $\begin{array}{l}\text { RSD } \\
(\%)\end{array}$ \\
\hline Fenoxycarb & 186 & 116 & 0.05 & 110 & 89 & 101 & 8 & 1 & 105 & 17 \\
\hline Fenpiclonil & 238 & 174 & 0.05 & 110 & 101 & 106 & 3 & 1 & 102 & 17 \\
\hline Fenpropathrin & 181 & 141 & 0.05 & 109 & 101 & 104 & 6 & 0 & 103 & 13 \\
\hline Fenpropimorph & 128 & 129 & 0.05 & 110 & 108 & 109 & 1 & 0 & 101 & 14 \\
\hline Fenvalerate & 167 & 125 & 0.25 & 110 & 102 & 103 & 2 & 5 & 98 & 15 \\
\hline Fipronil & 367 & 369 & 0.05 & 110 & 101 & 100 & 3 & 7 & 99 & 18 \\
\hline Flucythrinate & 199 & 157 & 0.05 & 110 & 102 & 106 & 3 & 1 & 103 & 15 \\
\hline Fludioxonil & 248 & 182 & 0.05 & 109 & 105 & 107 & 1 & 2 & 98 & 17 \\
\hline Flusilazole & 233 & 206 & 0.05 & 110 & 104 & 107 & 1 & 2 & 97 & 15 \\
\hline Flutolanil & 323 & 281 & 0.05 & 110 & 107 & 109 & 1 & 0 & 100 & 13 \\
\hline Flutriafol & 219 & 123 & 0.04 & 110 & 102 & 104 & 5 & 1 & 103 & 14 \\
\hline Fluvalinate, tau- & 250 & 252 & 0.15 & 110 & 97 & 99 & 5 & 6 & 99 & 15 \\
\hline Furalaxyl & 242 & 95 & 0.05 & 110 & 106 & 107 & 3 & 0 & 101 & 13 \\
\hline Heptenophos & 124 & 126 & 0.05 & 109 & 97 & 104 & 6 & 0 & 102 & 18 \\
\hline Hexaconazole & 216 & 214 & 0.05 & 110 & 106 & 108 & 1 & 1 & 102 & 14 \\
\hline Iprodione & 316 & 314 & 0.10 & 103 & 79 & 88 & 8 & 13 & 100 & 20 \\
\hline Jasmolin-1 & 164 & 123 & 0.04 & 110 & 92 & 104 & 4 & 2 & 97 & 15 \\
\hline Kresoxim-methyl & 116 & 206 & 0.05 & 109 & 106 & 109 & 0 & 1 & 100 & 15 \\
\hline Lindane & 183 & 219 & 0.05 & 110 & 107 & 110 & 0 & 0 & 99 & 15 \\
\hline Malathion & 173 & 127 & 0.05 & 108 & 103 & 107 & 3 & 0 & 104 & 17 \\
\hline Mecarbam & 329 & 131 & 0.05 & 110 & 109 & 110 & 0 & 0 & 101 & 15 \\
\hline Mepanipyrim & 223 & 222 & 0.05 & 110 & 88 & 91 & 7 & 12 & 85 & 19 \\
\hline Mepronil & 269 & 119 & 0.10 & 110 & 109 & 110 & 0 & 0 & 97 & 15 \\
\hline Metalaxyl & 206 & 160 & 0.05 & 107 & 105 & 108 & 2 & 0 & 103 & 12 \\
\hline Methidathion & 145 & 85 & 0.05 & 109 & 85 & 89 & 19 & 2 & 107 & 15 \\
\hline Metrafenone & 395 & 393 & 0.05 & 110 & 104 & 106 & 2 & 2 & 94 & 14 \\
\hline Mevinphos & 192 & 127 & 0.05 & 110 & 88 & 90 & 17 & 3 & 104 & 17 \\
\hline Myclobutanil & 179 & 150 & 0.05 & 110 & 102 & 107 & 2 & 1 & 98 & 15 \\
\hline Nitrothal-isopropyl & 236 & 254 & 0.05 & 110 & 108 & 108 & 1 & 1 & 99 & 13 \\
\hline Nuarimol & 235 & 203 & 0.05 & 110 & 108 & 110 & 0 & 0 & 101 & 15 \\
\hline Oxadixyl & 163 & 132 & 0.15 & 110 & 106 & 107 & 1 & 2 & 99 & 13 \\
\hline Parathion & 291 & 109 & 0.05 & 110 & 105 & 109 & 1 & 0 & 105 & 15 \\
\hline Parathion-methyl & 263 & 247 & 0.05 & 109 & 86 & 102 & 8 & 0 & 107 & 17 \\
\hline Penconazole & 159 & 248 & 0.05 & 109 & 108 & 110 & 0 & 0 & 100 & 15 \\
\hline Pentachloroaniline & 267 & 265 & 0.11 & 110 & 96 & 97 & 0 & 13 & 81 & 15 \\
\hline Pentachlorothioanisole & 296 & 246 & 0.05 & 110 & 87 & 89 & 0 & 21 & 77 & 16 \\
\hline Permethrin-cis & 183 & 163 & 0.05 & 110 & 108 & 110 & 0 & 0 & 101 & 14 \\
\hline Permethrin-trans & 183 & 163 & 0.05 & 110 & 106 & 107 & 3 & 0 & 100 & 13 \\
\hline Phenylphenol, 2- & 170 & 141 & 0.05 & 109 & 102 & 107 & 3 & 0 & 98 & 13 \\
\hline Phosalone & 182 & 184 & 0.05 & 110 & 90 & 92 & 13 & 5 & 101 & 19 \\
\hline Phosmet & 161 & 160 & 0.05 & 109 & 76 & 90 & 16 & 4 & 100 & 22 \\
\hline Phosphamidon & 264 & 127 & 0.05 & 110 & 91 & 94 & 13 & 3 & 103 & 19 \\
\hline Picoxystrobin & 335 & 145 & 0.05 & 110 & 105 & 109 & 1 & 0 & 103 & 12 \\
\hline Piperonyl-butoxide & 176 & 177 & 0.05 & 107 & 106 & 109 & 1 & 0 & 100 & 13 \\
\hline Pirimiphos-methyl & 276 & 305 & 0.05 & 110 & 109 & 109 & 1 & 0 & 102 & 13 \\
\hline Procymidone & 283 & 285 & 0.05 & 108 & 106 & 108 & 1 & 1 & 100 & 14 \\
\hline Profenofos & 337 & 206 & 0.05 & 108 & 93 & 102 & 8 & 0 & 104 & 17 \\
\hline Propargite & 173 & 135 & 0.33 & 109 & 104 & 109 & 1 & 0 & 103 & 16 \\
\hline Propiconazole & 259 & 261 & 0.05 & 109 & 106 & 107 & 2 & 1 & 99 & 14 \\
\hline Propyzamide & 173 & 175 & 0.05 & 110 & 107 & 108 & 2 & 0 & 102 & 12 \\
\hline Prothiofos & 309 & 267 & 0.05 & 110 & 108 & 109 & 1 & 0 & 99 & 13 \\
\hline Pyrazophos & 221 & 232 & 0.05 & 110 & 99 & 99 & 3 & 8 & 91 & 18 \\
\hline Pyrethrins & 123 & 160 & 0.36 & 110 & 87 & 103 & 7 & 0 & 105 & 18 \\
\hline Pyridaben & 147 & 148 & 0.05 & 110 & 107 & 107 & 1 & 2 & 99 & 14 \\
\hline
\end{tabular}


Table 7 (continued)

\begin{tabular}{|c|c|c|c|c|c|c|c|c|c|c|}
\hline Pesticide & $\begin{array}{l}\text { Quan. } \\
\text { ion } m / z\end{array}$ & $\begin{array}{l}\text { Qual. } \\
\text { ion } m / z\end{array}$ & $\begin{array}{l}\text { Fortification } \\
\text { level } \\
\left(\mathrm{mg} \mathrm{kg}^{-1}\right)\end{array}$ & $\begin{array}{l}\text { \# QCs matrices } \\
\text { (see Table 6) }\end{array}$ & $\begin{array}{l}\text { Both diagn. } \\
\text { ions } 60-140 \%\end{array}$ & $\begin{array}{l}\text { One of diagn. } \\
\text { ions } 60-140 \%\end{array}$ & $\begin{array}{l}\text { Both diagn. } \\
\text { ions }>140 \%\end{array}$ & $\begin{array}{l}\text { Both diagn. } \\
\text { ions }<60 \%\end{array}$ & $\begin{array}{l}\text { Average } \\
\text { recov. (\%) } \\
\text { Quan. ion }\end{array}$ & $\begin{array}{l}\text { RSD } \\
(\%)\end{array}$ \\
\hline Pyridaphenthion & 340 & 199 & 0.05 & 110 & 96 & 101 & 7 & 2 & 102 & 17 \\
\hline Pyrifenox & 262 & 264 & 0.05 & 110 & 108 & 110 & 0 & 0 & 100 & 15 \\
\hline Pyrimethanil & 199 & 198 & 0.05 & 110 & 107 & 106 & 1 & 3 & 90 & 14 \\
\hline Pyriproxyfen & 226 & 136 & 0.05 & 110 & 104 & 107 & 2 & 1 & 103 & 16 \\
\hline Quinalphos & 157 & 146 & 0.05 & 110 & 104 & 105 & 4 & 1 & 104 & 14 \\
\hline Quinoxyfen & 307 & 272 & 0.05 & 110 & 106 & 106 & 0 & 4 & 92 & 14 \\
\hline Quintozene & 237 & 142 & 0.05 & 110 & 107 & 107 & 1 & 2 & 93 & 16 \\
\hline Silafluofen & 179 & 286 & 0.05 & 110 & 106 & 106 & 0 & 4 & 98 & 14 \\
\hline Spirodiclofen & 312 & 314 & 0.25 & 110 & 95 & 96 & 6 & 8 & 96 & 19 \\
\hline Spiromesifen & 272 & 254 & 0.05 & 110 & 105 & 108 & 1 & 1 & 96 & 16 \\
\hline Spiroxamine & 100 & 198 & 0.10 & 110 & 107 & 109 & 0 & 1 & 96 & 13 \\
\hline TDE, $p, p^{\prime}-$ & 235 & 237 & 0.05 & 110 & 97 & 100 & 5 & 5 & 103 & 14 \\
\hline Tebuconazole & 250 & 252 & 0.15 & 67 & 66 & 67 & 0 & 1 & 97 & 15 \\
\hline Tebufenpyrad & 171 & 318 & 0.05 & 110 & 107 & 108 & 1 & 1 & 100 & 13 \\
\hline Tebupirimfos & 234 & 318 & 0.05 & 110 & 108 & 109 & 1 & 0 & 101 & 14 \\
\hline Tefluthrin & 177 & 197 & 0.05 & 110 & 106 & 107 & 3 & 0 & 103 & 13 \\
\hline Tetraconazole & 336 & 338 & 0.05 & 110 & 109 & 109 & 1 & 0 & 99 & 14 \\
\hline Tetradifon & 356 & 229 & 0.15 & 109 & 109 & 110 & 0 & 0 & 99 & 14 \\
\hline Thiometon & 88 & 125 & 0.05 & 110 & 108 & 110 & 0 & 0 & 104 & 15 \\
\hline Tolclofos-methyl & 265 & 267 & 0.05 & 108 & 107 & 107 & 2 & 0 & 101 & 13 \\
\hline Tri-allate & 268 & 270 & 0.05 & 110 & 104 & 105 & 4 & 1 & 104 & 13 \\
\hline Triazamate & 242 & 227 & 0.05 & 110 & 107 & 107 & 3 & 0 & 102 & 14 \\
\hline Triazophos & 285 & 257 & 0.05 & 109 & 95 & 100 & 8 & 2 & 104 & 18 \\
\hline Trifloxystrobin & 131 & 116 & 0.05 & 110 & 108 & 109 & 1 & 0 & 103 & 14 \\
\hline Triflumizole & 278 & 287 & 0.03 & 110 & 105 & 107 & 0 & 3 & 99 & 15 \\
\hline Trifluralin & 264 & 306 & 0.05 & 110 & 107 & 107 & 2 & 1 & 101 & 14 \\
\hline Vinclozolin & 212 & 198 & 0.05 & 107 & 106 & 109 & 1 & 0 & 103 & 11 \\
\hline Total & & & & 14696 & 13688 & 14057 & 402 & 300 & & \\
\hline$\%$ of \# QCs & & & & & 93.1 & 95.2 & 2.7 & 2.0 & & \\
\hline
\end{tabular}

without re-evaluating the real need for it. Reconstitution was performed by first dissolving in methanol (ultrasonication) and then dilution with LC mobile phase component A.

\section{Validation of $L C-M S-M S$ method}

The LC-MS-MS method was validated in three separate studies, one using the API2000 with injection of $20 \mathrm{mg}$ matrix equivalent and the other two using the API3000 with injection of $2 \mathrm{mg}$ matrix equivalent. A total of 140 pesticides and degradation products were included. In contrast with the full-scan acquisition in GC-MS, in LC-MS-MS data were acquired for a fixed, limited, set of pesticides. Although many pesticides from the GC-MS method can also be analyzed by LC-MS-MS, emphasis was on pesticides that were not, or less, amenable to GC analysis.

Recovery, based on matrix-matched calibration, and repeatability were evaluated at the 0.01 and $0.1 \mathrm{mg} \mathrm{kg}^{-1}$ level for vegetable and fruit matrices; the results are listed in Table 8. Although acceptable performance data were obtained for most of the pesticides, low recovery and/or high variability were observed for some. Among these were compounds that were also reported as troublesome by other workers using alternative multi-residue methods, e.g. asulam [30]. Low recovery could be partly attributed to poor extraction efficiency (asulam, hymexazol, and, in orange, propamocarb) or degradation during sample preparation (cycloxydim, sethoxydim, profoxydim, tepraloxydim, dichlofluanide, tolylfluanide, thiodicarb, thiophanate-methyl, and, in lettuce, disulfoton and furathiocarb). The degradation seems to be related to the change of solvent, as is apparent from comparison of GC-MS and LC-MS-MS validation data for dichlofluanide, tolylfluanide, and disulfoton. Fortunately, for many of these the degradation products formed are also part of the residue definition and are included in the method. Indeed, elevated recovery was observed for the degradation products when determined in the same validation set as the parent compound. In the analysis, therefore, degradation is not necessarily a problem, because the results (expressed as defined in the residue definition) have to be summed. In routine analytical quality control (see below) the data were evaluated this way. 


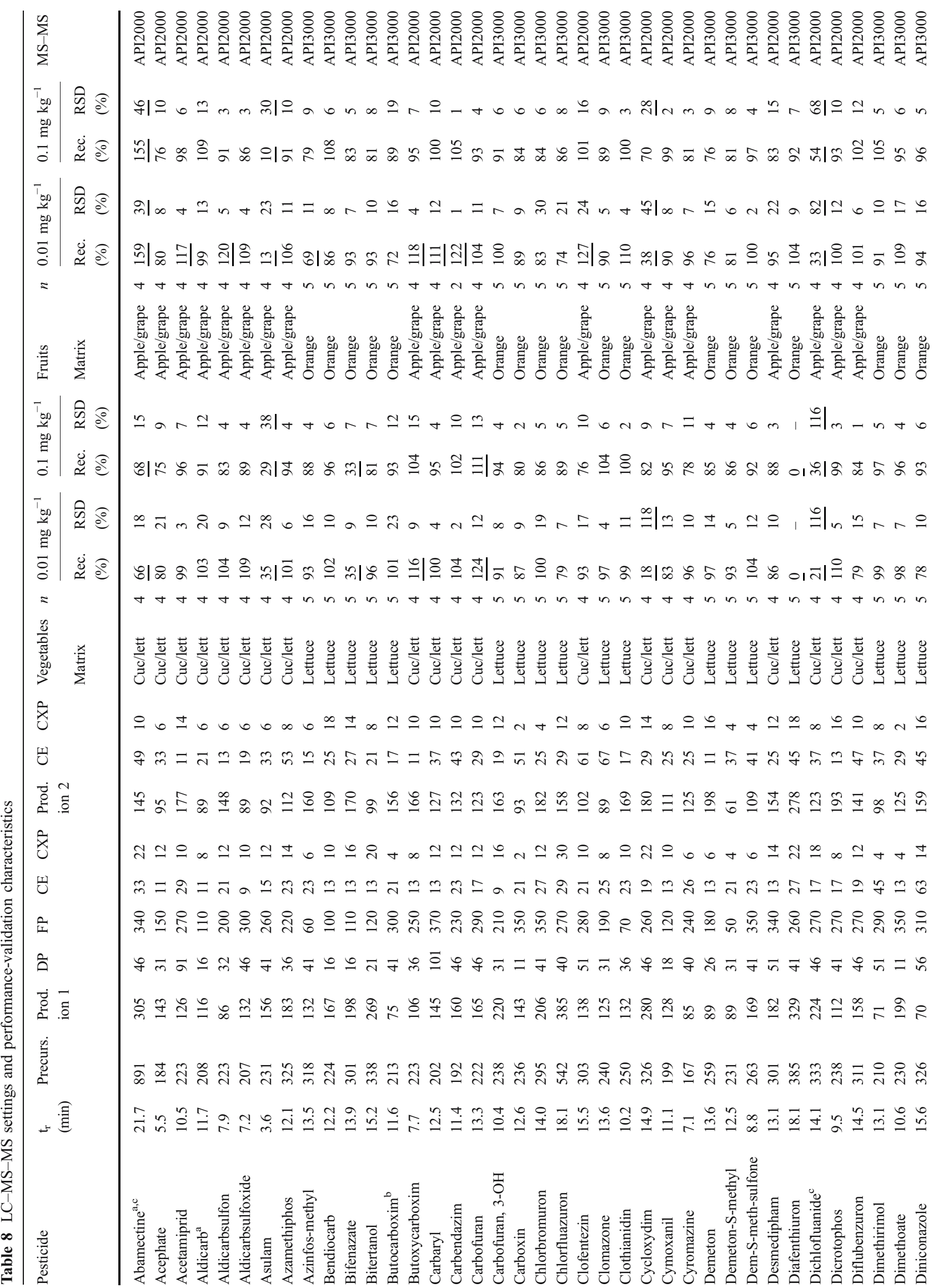




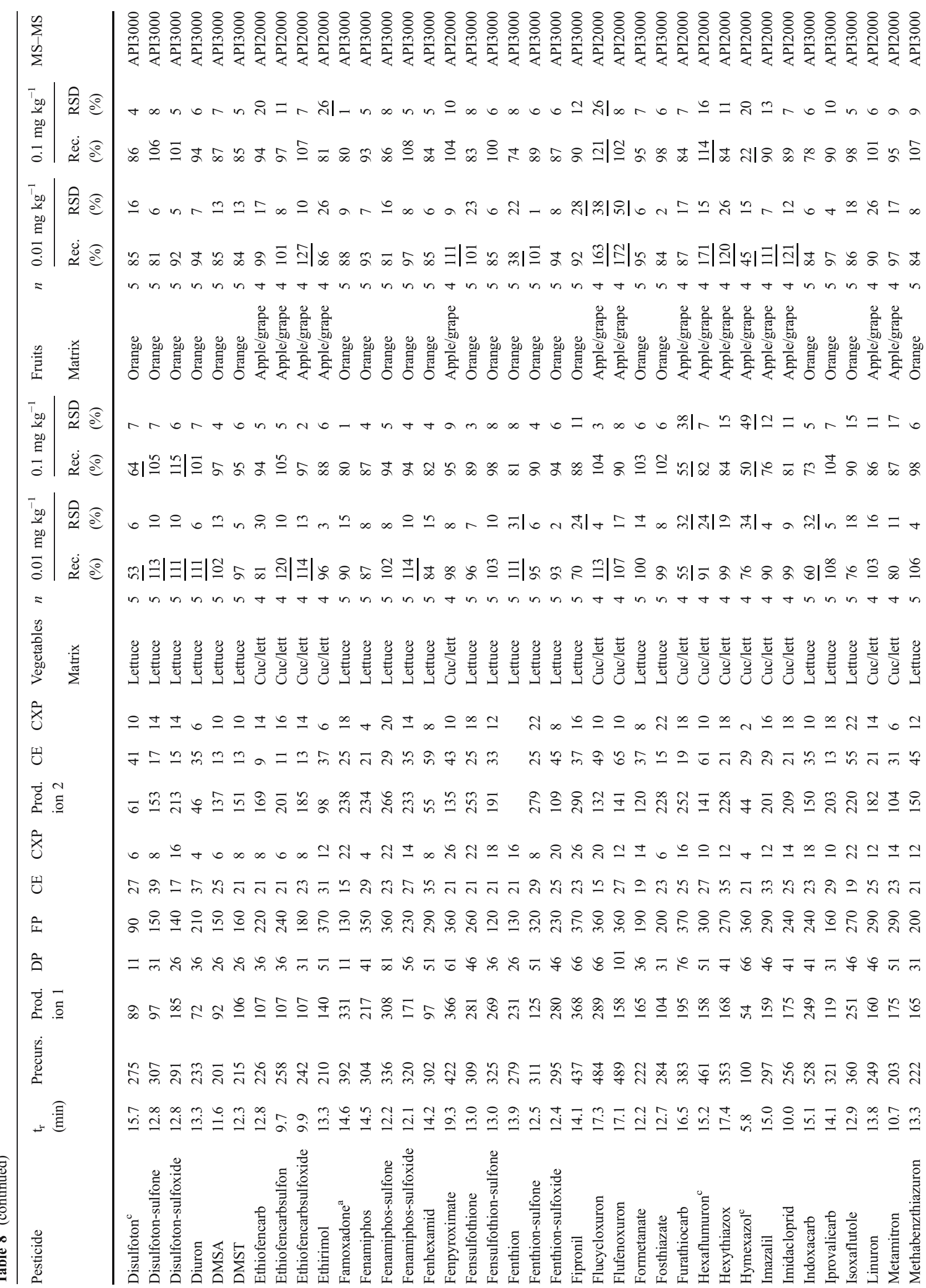






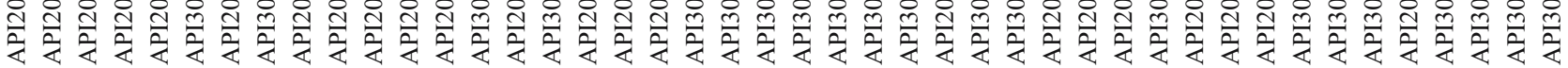
$n-6$ n

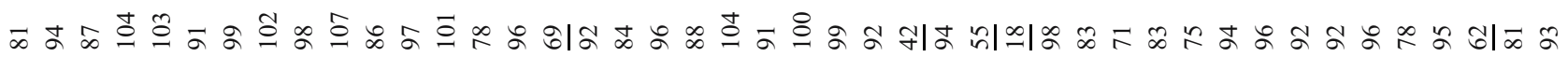

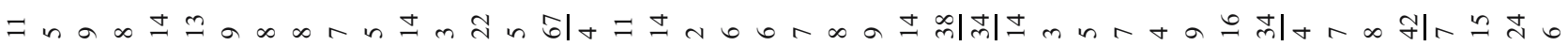



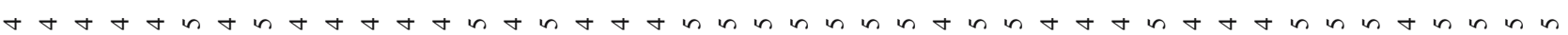

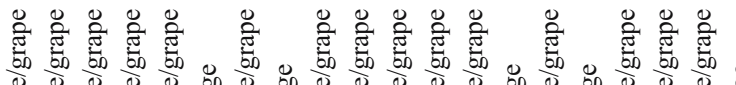


I+

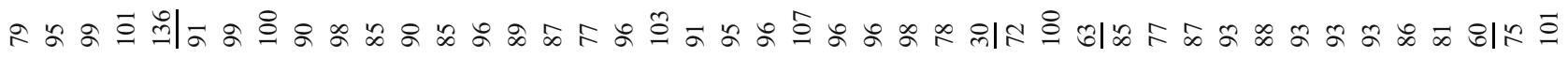
므ำก ๓



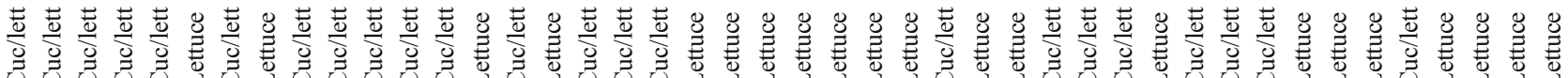

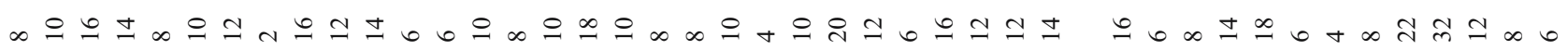



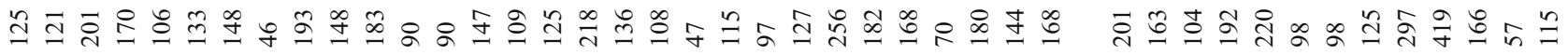
。

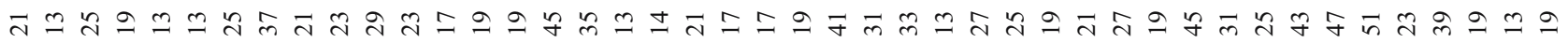

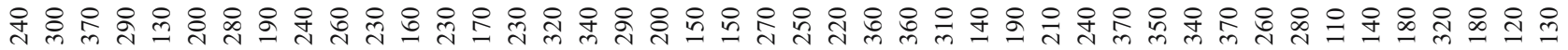


แ

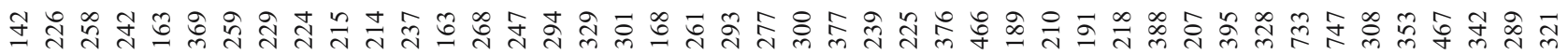

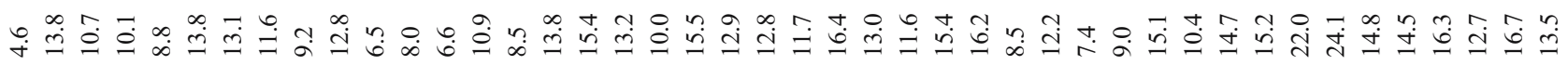






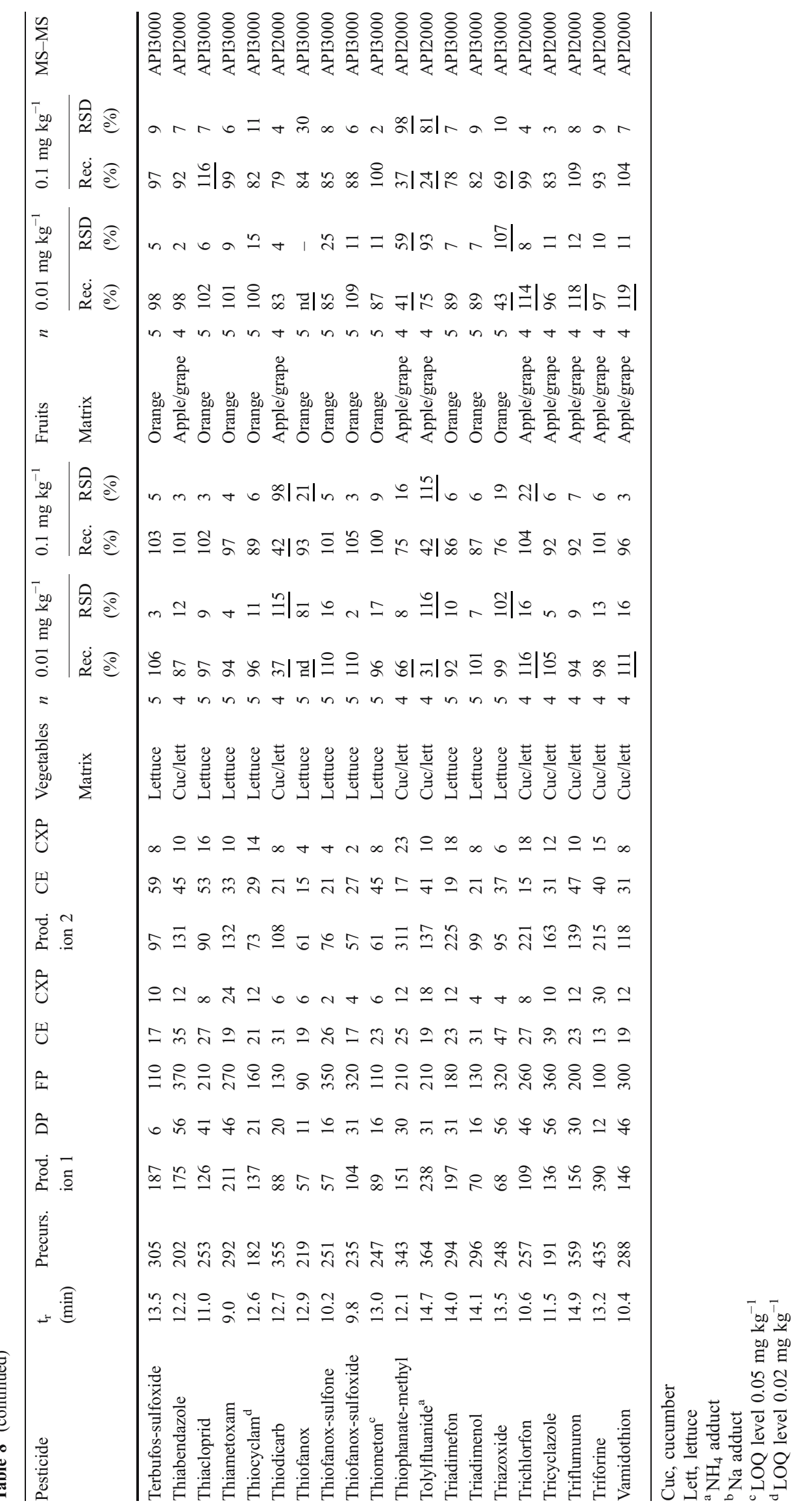




\section{Analytical quality-control data from routine} $L C-M S-M S$ analysis

In the same way as for GC-MS analysis, the initial validation data are continually being supplemented by performance data generated as part of analytical quality control during routine analysis of samples. With each set of analytical samples at least one was fortified with the full quantitative suite (i.e. 136 pesticides and degradation products) at the $0.05 \mathrm{mg} \mathrm{kg}^{-1}$ level. A compilation was made from all the data generated over a period of 12 months, which included data for more than one hundred vegetable and fruit matrices. A limited number of dry matrices (flour, milk powder) were also included in the set. The data were evaluated for one transition for each pesticide, using the API3000 and injection of $2 \mathrm{mg}$ equivalent of matrix $\left(10 \mu \mathrm{L}\right.$ of a $0.2 \mathrm{~g} \mathrm{~mL}^{-1}$ extract $)$. Examples of typical extracted ion chromatograms are shown in Fig. 5.

For all fortified samples the matrix effect was also established by analyzing the corresponding matrixmatched standard, at the same level as in the extract of the fortified sample, against a solvent standard. Suppression (or enhancement) of up to $20 \%$ was regarded as acceptable for quantification. The number of compounds for which the response in matrix relative to that in solvent was between 80 and $120 \%$ is given in Table 9 for each matrix. Whereas for beetroot, asparagus, and kangkung little or no matrix effects exceeding 20\% were observed, such effects were much more common for herbs and citrus fruits.

In contrast with GC, for which matrix effects are mainly caused by shielding of active sites in the inlet and were, to some extent predictable (in relation to the matrix load injected and the lability and/or polarity of analyte), in LCMS-MS matrix effects are much less predictable. Although they do depend on the amount of matrix introduced into the system, and also tend to be more abundant in complex ("aromatic") matrices, it cannot be readily predicted for which pesticides the effects occur. For this reason use of one matrix-matched standard as representative calibrant for a whole range of commodities, which worked reasonably well in GC-MS analysis, was not feasible in LC-MS-MS analysis. Consequently, critical evaluation of the matrix
Fig. 5 Typical extracted ion chromatograms obtained by LC-MS-MS analysis of vegetable and fruit extracts (calibration standard in mango matrix, $10 \mathrm{pg} \mu \mathrm{L}$, corresponding to $0.05 \mathrm{mg} \mathrm{kg}^{-1}$ )

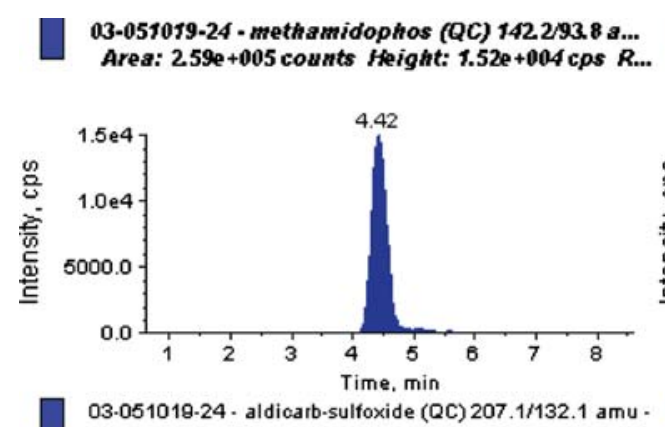

03-051019-24 - aldicarb-sulfoxide (QC) 207.1/132.1 amu -

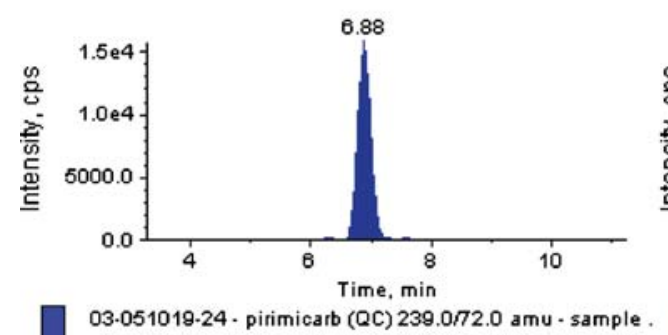

$03.051019 \cdot 24$ - pirimicarb (QC) $239.0 / 72.0$ amu - sample.
Area: $4.69 \mathrm{e}+005$ counts Height: $4.15 \mathrm{e}+004 \mathrm{cps}$ RT: $13 .$.

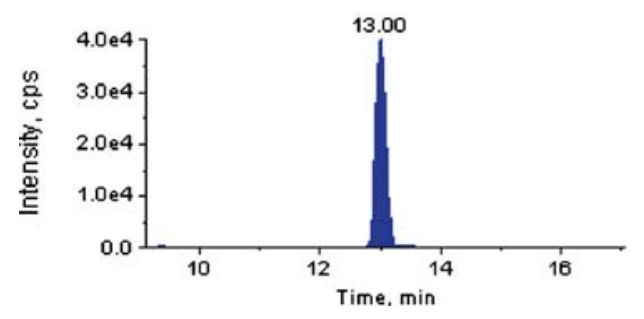

03-051019-24 - cyromazine (QC) 167.4/85.1 amu - sampl.. Area: $3.33 \mathrm{e}+005$ counts Height: $9.24 \mathrm{e}+003$ cps RT: 6.8 .

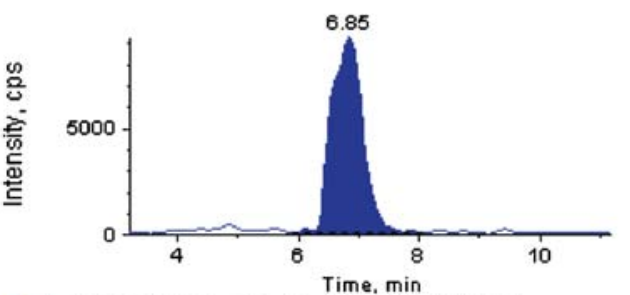

03-051019-24 - imidacloprid (QC) 256.1/175.1 amu - sam. Area: $6.51 \mathrm{e}+004$ counts Height: $5.85 \mathrm{e}+003$ cps RT: 9.9 .

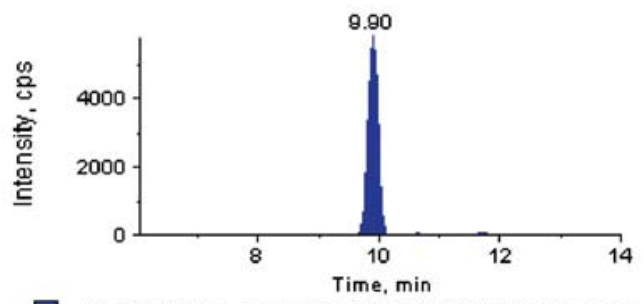

03.051019-24 - abamectin (QC) 890.6/305.2 amu - sampl. Area: $2.50 \mathrm{e}+004$ counts Height: $1.27 \mathrm{e}+003$ cps RT: 21 .




Table 9 (continued)

$N \quad$ Matrix effects

Rel. resp. $80-120 \%>20 \%$ suppr. $>20 \%$ enhanc. $n^{*}$

\# Pesticides

Calc. using solvent std

\begin{tabular}{|c|c|c|c|c|c|c|c|c|c|c|c|}
\hline Cucumber & 135 & 110 & 15 & 10 & 104 & 99 & 5 & 0 & 101 & 3 & 0 \\
\hline Horseradish powder & 135 & 110 & 15 & 10 & 104 & 88 & 11 & 5 & 97 & 5 & 2 \\
\hline Tarrragon $(2 / 2)$ & 135 & 110 & 8 & 17 & 104 & 96 & 4 & 4 & 94 & 6 & 4 \\
\hline Avocado $(1 / 2)$ & 135 & 109 & 22 & 4 & 104 & 81 & 21 & 2 & 90 & 13 & 1 \\
\hline Haricot bean & 135 & 109 & 25 & 1 & 104 & 83 & 20 & 1 & 90 & 13 & 1 \\
\hline Kiwi & 135 & 109 & 10 & 16 & 104 & 97 & 6 & 1 & 100 & 2 & 2 \\
\hline Peach $(12 / 2)$ & 135 & 108 & 24 & 3 & 104 & 88 & 14 & 2 & 93 & 9 & 2 \\
\hline Raspberry (3/3) & 135 & 107 & 26 & 2 & 104 & 80 & 22 & 2 & 90 & 11 & 3 \\
\hline Blackberry & 133 & 106 & 17 & 10 & 102 & 91 & 10 & 1 & 91 & 9 & 2 \\
\hline Diced pumpkins & 135 & 106 & 27 & 2 & 104 & 95 & 8 & 1 & 100 & 3 & 1 \\
\hline Plum $(2 / 3)$ & 135 & 106 & 23 & 6 & 104 & 86 & 18 & 0 & 85 & 18 & 1 \\
\hline Yam & 135 & 106 & 1 & 28 & 104 & 97 & 6 & 1 & 96 & 8 & 0 \\
\hline Avocado $(2 / 2)$ & 134 & 103 & 29 & 2 & 103 & 68 & 34 & 1 & 80 & 22 & 1 \\
\hline Dill leaves & 135 & 103 & 15 & 17 & 104 & 94 & 7 & 3 & 93 & 9 & 2 \\
\hline Honey & 106 & 103 & 3 & 0 & 82 & 82 & 0 & 0 & 82 & 0 & 0 \\
\hline Chervil & 135 & 102 & 29 & 4 & 104 & 95 & 9 & 0 & 98 & 5 & 1 \\
\hline Parsley & 135 & 102 & 29 & 4 & 104 & 95 & 4 & 5 & 99 & 1 & 4 \\
\hline Nectarine & 134 & 101 & 29 & 4 & 103 & 92 & 8 & 3 & 98 & 4 & 1 \\
\hline Bean sprouts & 106 & 100 & 5 & 1 & 82 & 76 & 6 & 0 & 78 & 4 & 0 \\
\hline Sweetcorn $(1 / 2)$ & 106 & 99 & 6 & 1 & 82 & 76 & 5 & 1 & 77 & 3 & 2 \\
\hline Beetroot leaves & 135 & 98 & 32 & 5 & 104 & 85 & 19 & 0 & 99 & 5 & 0 \\
\hline Chestnuts & 106 & 98 & 1 & 7 & 82 & 76 & 4 & 2 & 79 & 3 & 0 \\
\hline Pomegranate $(1 / 2)$ & 135 & 97 & 37 & 1 & 104 & 84 & 20 & 0 & 100 & 4 & 0 \\
\hline Pomegranate $(2 / 2)$ & 135 & 97 & 37 & 1 & 104 & 84 & 20 & 0 & 100 & 4 & 0 \\
\hline Pear syrup & 106 & 95 & 3 & 8 & 82 & 79 & 3 & 0 & 80 & 2 & 0 \\
\hline Alfalfa & 106 & 94 & 11 & 1 & 82 & 75 & 7 & 0 & 78 & 4 & 0 \\
\hline Fennel leaves $(1 / 2)$ & 106 & 92 & 8 & 6 & 82 & 74 & 5 & 3 & 76 & 2 & 4 \\
\hline Chili pepper & 135 & 91 & 40 & 4 & 104 & 95 & 8 & 1 & 101 & 1 & 2 \\
\hline Turnip tops & 106 & 90 & 15 & 1 & 82 & 76 & 2 & 4 & 78 & 0 & 4 \\
\hline Blueberry & 135 & 89 & 43 & 3 & 102 & 66 & 36 & 0 & 91 & 11 & 0 \\
\hline Litchi & 135 & 88 & 45 & 2 & 104 & 78 & 26 & 0 & 99 & 4 & 1 \\
\hline Salak & 135 & 88 & 42 & 5 & 104 & 82 & 20 & 2 & 99 & 4 & 1 \\
\hline Pepper powder & 106 & 87 & 16 & 3 & 82 & 54 & 27 & 1 & 70 & 11 & 1 \\
\hline Celery leaves $(2 / 3)$ & 135 & 85 & 41 & 9 & 104 & 93 & 10 & 1 & 100 & 2 & 2 \\
\hline Lemon & 134 & 84 & 47 & 3 & 104 & 78 & 20 & 6 & 97 & 3 & 4 \\
\hline Physalis & 135 & 83 & 48 & 4 & 104 & 71 & 33 & 0 & 99 & 5 & 0 \\
\hline Maize (feed) & 135 & 81 & 53 & 1 & 104 & 95 & 6 & 3 & 93 & 3 & 8 \\
\hline Sweetcorn $(2 / 2)$ & 135 & 80 & 50 & 5 & 104 & 79 & 22 & 3 & 98 & 6 & 0 \\
\hline Coriander $(1 / 2)$ & 135 & 79 & 56 & 0 & 104 & 68 & 34 & 2 & 95 & 6 & 3 \\
\hline Mangostan & 135 & 76 & 40 & 19 & 104 & 46 & 54 & 4 & 69 & 35 & 0 \\
\hline Celery leaves $(3 / 3)$ & 134 & 75 & 58 & 1 & 103 & 86 & 16 & 1 & 99 & 2 & 2 \\
\hline Laos & 135 & 73 & 57 & 5 & 104 & 70 & 33 & 1 & 99 & 4 & 1 \\
\hline Chives & 135 & 71 & 57 & 7 & 104 & 98 & 5 & 1 & 102 & 1 & 1 \\
\hline Coriander $(2 / 2)$ & 135 & 65 & 60 & 10 & 104 & 83 & 21 & 0 & 98 & 6 & 0 \\
\hline Tea (black) & 136 & 65 & 69 & 2 & 104 & 60 & 43 & 1 & 87 & 14 & 3 \\
\hline Lemon puree & 135 & 53 & 80 & 2 & 104 & 68 & 36 & 0 & 103 & 1 & 0 \\
\hline Ginger & 135 & 46 & 86 & 3 & 104 & 68 & 34 & 2 & 98 & 3 & 3 \\
\hline Grapefruit $(1 / 2)$ & 133 & 46 & 87 & 0 & 102 & 43 & 59 & 0 & 98 & 1 & 3 \\
\hline
\end{tabular}


Table 9 (continued)

\begin{tabular}{|c|c|c|c|c|c|c|c|c|c|c|c|}
\hline & \multirow[t]{4}{*}{$N$} & \multicolumn{3}{|l|}{ Matrix effects } & \multirow[t]{4}{*}{$n^{*}$} & \multirow{2}{*}{\multicolumn{6}{|c|}{$\frac{\text { Recovery }}{\text { \# Pesticides }}$}} \\
\hline & & \multicolumn{3}{|l|}{ \# Pesticides } & & & & & & & \\
\hline & & \multirow[t]{2}{*}{ Rel. resp. $80-120 \%$} & \multirow[t]{2}{*}{$>20 \%$ suppr. } & \multirow[t]{2}{*}{$>20 \%$ enhanc. } & & \multicolumn{3}{|c|}{ Calc. using solvent std } & \multicolumn{3}{|c|}{$\begin{array}{l}\text { Calc. using matrix-matched } \\
\text { std }\end{array}$} \\
\hline & & & & & & $60-140 \%$ & $<60 \%$ & $>140 \%$ & $60-140 \%$ & $<60 \%$ & $>140 \%$ \\
\hline Grapefruit $(2 / 2)$ & 135 & 46 & 88 & 1 & 103 & 61 & 41 & 1 & 97 & 3 & 3 \\
\hline Oregano & 135 & 46 & 75 & 14 & 104 & 52 & 50 & 2 & 87 & 16 & 1 \\
\hline Kumquat & 135 & 38 & 95 & 2 & 104 & 47 & 56 & 1 & 94 & 6 & 4 \\
\hline Lime & 134 & 38 & 94 & 2 & 103 & 48 & 52 & 3 & 96 & 4 & 3 \\
\hline Tarrragon $(1 / 2)$ & 135 & 38 & 95 & 2 & 104 & 41 & 63 & 0 & 90 & 13 & 1 \\
\hline Italian herb mix & 135 & 33 & 101 & 1 & 104 & 54 & 49 & 1 & 95 & 8 & 1 \\
\hline Total QC results & 13497 & 10488 & 2566 & 443 & 10395 & 8618 & 1613 & 164 & 9533 & 708 & 154 \\
\hline Percentage of total results & & 78 & 19 & 3 & & 83 & 16 & 2 & 92 & 7 & 1 \\
\hline
\end{tabular}

${ }^{\text {a }}$ Recovery at $0.05 \mathrm{mg} \mathrm{kg}^{-1}$ (higher for seven pesticides). The pesticides included are listed in Table 10

$N$ is the total number of individual compounds (pesticides and metabolites) added to the matrix

$n^{*}$ is the total number of pesticides added to the matrix. Compounds belonging to the same residue definition counted as one

effect was required; if unacceptable suppression occurred there was no alternative to quantification by use of the appropriate matrix-matched calibration standard or, when not available, by standard addition.

Recovery of the pesticides from the fortified samples was calculated relative to that from a solvent standard and a matrix-matched standard and tested against the $60-140 \%$ criterion for evaluation of routine analytical quality-control samples [37]. A total of more than 10,000 recovery values were evaluated. Without matrix-matched calibration, acceptable recovery was obtained for $83 \%$ of the pesticides. Deviating recoveries were usually too low, mainly because of ion suppression, as is apparent from the results obtained from determination of recovery using matrix-matched calibration, for which $92 \%$ met the criterion.

Concentrating on performance at the pesticide level (Table 10) enables easy identification of troublesome pesticides. All compounds belonging to the same residue definition were summed (according to the residue definition) and counted as one, thereby compensating for possible conversion during sample pretreatment. This way the low recovery of dichlofluanide and the corresponding high recovery of DMSA were acceptable for most matrices because recovery for the sum met the criterion. Pesticides for which multi-matrix analysis under fixed conditions was less favorable included asulam, bifenazate, cyromazine, furathiocarb, propamocarb, pymetrozine, and thiocyclam (low recovery because of varying extraction efficiency and/or degradation). As already observed during validation, the method was also less suitable for cycloxydim, profoxydim, sethoxydim, and tepraloxydim. For these compounds recovery was too high, possibly because of degradation in the calibration standard used for preparation of the matrixmatched standards.

Averaging acceptable recoveries reveals there is some bias, because the values are mostly approximately $87 \%$ (in contrast with the GC-MS data, for which the average was approximately $100 \%$ ). It was noted that for dry crops relatively low recovery (typically between $60-70 \%$ ) was obtained for all pesticides. The cause is not clear. This bias can also be seen in tables in other papers (barley [26], soya grain [33]).

Independent evaluation of method performance by proficiency testing

From results obtained over the years from participation in proficiency tests, an additional and independent verification of method performance could be made. The data are summarized in Table 11 and clearly show that good quantitative data were consistently obtained from both GC-MS and LC-MS-MS, with method performance good $(Z$-score $<2)$ 54 times, doubtful $(2<Z<3)$ three times, and never poor. It also shows that the calibration approach (one-point calibration, tomato-matrix standard for GC and matrix-matched standard for LC) is fit-for-purpose.

\section{Conclusions}

The ethyl acetate-based multi-residue method has been modified to meet today's demands in respect of ease and speed of sample preparation. For GC-MS analysis, com- 
Table 10 Recovery over all matrices (LC-MS-MS)

\begin{tabular}{|c|c|c|c|c|c|c|c|}
\hline & & \# ACQ samples & \# Recov. $60-140 \%$ & \# Recov. $<60 \%$ & \# Recov. $>140 \%$ & Average recov. $(\%)^{\mathrm{a}}$ & $\operatorname{RSD}(\%)^{\mathrm{a}}$ \\
\hline 1 & Abamectin & 102 & 100 & 2 & $\mathbf{0}$ & 86 & 17 \\
\hline 2 & Acephate & 102 & 93 & 9 & $\mathbf{0}$ & 78 & 13 \\
\hline \multirow[t]{4}{*}{3} & Acetamiprid & 102 & 97 & 5 & $\mathbf{0}$ & 90 & 11 \\
\hline & Aldicarb & 102 & 101 & 0 & 1 & 91 & 13 \\
\hline & Aldicarb-sulfone & 102 & 102 & 0 & 0 & 92 & 12 \\
\hline & Aldicarb-sulfoxide & 102 & 96 & 6 & 0 & 84 & 13 \\
\hline 4 & Asulam & 102 & 69 & 32 & 1 & 85 & 17 \\
\hline 5 & Azamethiphos & 102 & 102 & $\mathbf{0}$ & $\mathbf{0}$ & 89 & 12 \\
\hline 6 & Azinfos-methyl & 102 & 96 & 5 & 1 & 87 & 15 \\
\hline 7 & Bendiocarb & 93 & 93 & $\mathbf{0}$ & $\mathbf{0}$ & 88 & 12 \\
\hline 8 & Bifenazate & 98 & 60 & 37 & 1 & 85 & 18 \\
\hline \multirow[t]{3}{*}{9} & Bitertanol & 102 & 98 & 4 & $\mathbf{0}$ & 84 & 15 \\
\hline & Butocarboxim & 102 & 101 & 1 & 0 & 88 & 14 \\
\hline & Butoxycarboxim & 102 & 101 & 1 & 0 & 91 & 12 \\
\hline \multirow[t]{4}{*}{10} & Carbaryl & 102 & 100 & 1 & 1 & 87 & 13 \\
\hline & Carbendazim & 100 & 97 & 2 & 1 & 93 & 14 \\
\hline & Carbofuran & 102 & 100 & 1 & 1 & 92 & 12 \\
\hline & Carbofuran,3-hydroxy- & 102 & 102 & 0 & 0 & 93 & 11 \\
\hline 11 & Carboxin & 102 & 97 & 5 & $\mathbf{0}$ & 84 & 13 \\
\hline 12 & Chlorbromuron & 102 & 98 & 4 & $\mathbf{0}$ & 86 & 14 \\
\hline 13 & Chlorfluazuron & 102 & 93 & 8 & 1 & 87 & 15 \\
\hline 14 & Clofentezine & 102 & 89 & 13 & $\mathbf{0}$ & 80 & 15 \\
\hline 15 & Clomazone & 93 & 89 & 3 & 1 & 85 & 12 \\
\hline 16 & Clothianidin & 93 & 91 & 2 & $\mathbf{0}$ & 91 & 12 \\
\hline 17 & Cycloxydim & 102 & 68 & 11 & 23 & 104 & 19 \\
\hline 18 & Cymoxanil & 102 & 102 & $\mathbf{0}$ & $\mathbf{0}$ & 91 & 15 \\
\hline 19 & Cyromazine & 102 & 49 & 53 & $\mathbf{0}$ & 74 & 12 \\
\hline \multirow[t]{3}{*}{20} & Demeton & 102 & 102 & $\mathbf{0}$ & $\mathbf{0}$ & 89 & 14 \\
\hline & Demeton-S-methyl & 102 & 100 & 2 & 0 & 87 & 14 \\
\hline & Demeton-S-methylsulfone & 102 & 101 & 1 & 0 & 91 & 12 \\
\hline \multirow[t]{2}{*}{21} & Desmedipham & 102 & 96 & 6 & $\mathbf{0}$ & 83 & 14 \\
\hline & Dichlofluanid & 102 & 36 & 66 & 0 & 80 & 19 \\
\hline 22 & Dicrotophos & 102 & 100 & 2 & $\mathbf{0}$ & 89 & 14 \\
\hline 23 & Diflubenzuron & 102 & 98 & 4 & $\mathbf{0}$ & 82 & 15 \\
\hline \multirow[t]{2}{*}{24} & Dimethirimol & 93 & 90 & 3 & $\mathbf{0}$ & 89 & 11 \\
\hline & Dimethoate & 102 & 101 & 1 & 0 & 90 & 12 \\
\hline \multirow[t]{4}{*}{25} & Diniconazole & 93 & 84 & 8 & 1 & 86 & 16 \\
\hline & Disulfoton & 93 & 67 & 25 & 1 & 75 & 13 \\
\hline & Disulfoton-sulfone & 93 & 93 & 0 & 0 & 88 & 12 \\
\hline & Disulfoton-sulfoxide & 93 & 89 & 0 & 4 & 96 & 16 \\
\hline \multirow[t]{6}{*}{26} & Diuron & 93 & 92 & 1 & $\mathbf{0}$ & 87 & 14 \\
\hline & DMSA & 102 & 41 & 0 & 61 & 109 & 17 \\
\hline & DMST & 102 & 96 & 1 & 5 & 104 & 16 \\
\hline & Ethiofencarb & 102 & 99 & 3 & 0 & 86 & 14 \\
\hline & Ethiofencarb-sulfone & 102 & 102 & 0 & 0 & 90 & 13 \\
\hline & Ethiofencarb-sulfoxide & 102 & 101 & 1 & 0 & 92 & 15 \\
\hline 27 & Ethirimol & 102 & 98 & 4 & $\mathbf{0}$ & 88 & 12 \\
\hline \multirow[t]{4}{*}{28} & Famoxadone & 102 & 95 & 7 & $\mathbf{0}$ & 83 & 14 \\
\hline & Fenamiphos & 102 & 100 & 2 & 0 & 89 & 14 \\
\hline & Fenamiphos-sulfone & 102 & 102 & 0 & 0 & 91 & 12 \\
\hline & Fenamiphos-sulfoxide & 93 & 92 & 1 & 0 & 90 & 11 \\
\hline 29 & Fenhexamid & 102 & 96 & 6 & $\mathbf{0}$ & 85 & 12 \\
\hline \multirow[t]{4}{*}{30} & Fenpyroximate & 102 & 92 & 10 & $\mathbf{0}$ & 87 & 13 \\
\hline & Fensulfothion & 102 & 102 & 0 & 0 & 88 & 11 \\
\hline & Fensulfothion-sulfone & 93 & 91 & 2 & 0 & 85 & 12 \\
\hline & Fenthion & 102 & 99 & 3 & 0 & 87 & 14 \\
\hline
\end{tabular}


Table 10 (continued)

\begin{tabular}{|c|c|c|c|c|c|c|c|}
\hline & & \# ACQ samples & \# Recov. $60-140 \%$ & \# Recov. $<60 \%$ & \# Recov. $>140 \%$ & Average recov. $(\%)^{\mathrm{a}}$ & $\operatorname{RSD}(\%)^{\mathrm{a}}$ \\
\hline & Fenthion-sulfone & 102 & 99 & 2 & 1 & 88 & 15 \\
\hline & Fenthion-sulfoxide & 102 & 102 & 0 & 0 & 93 & 14 \\
\hline 31 & Flucycloxuron & 102 & 94 & 8 & $\mathbf{0}$ & 88 & 15 \\
\hline 32 & Flufenoxuron & 102 & 93 & 9 & $\mathbf{0}$ & 87 & 14 \\
\hline 33 & Fosthiazate & 93 & 93 & $\mathbf{0}$ & $\mathbf{0}$ & 90 & 12 \\
\hline 34 & Furathiocarb & 102 & 79 & 20 & 3 & 84 & 16 \\
\hline 35 & Hexaflumuron & 102 & 90 & 10 & 2 & 85 & 18 \\
\hline 36 & Hexythiazox & 102 & 91 & 11 & $\mathbf{0}$ & 85 & 15 \\
\hline 37 & Imazalil & 101 & 92 & 9 & $\mathbf{0}$ & 83 & 14 \\
\hline 38 & Imidacloprid & 102 & 99 & 3 & $\mathbf{0}$ & 90 & 14 \\
\hline 39 & Indoxacarb & 101 & 96 & 5 & $\mathbf{0}$ & 86 & 16 \\
\hline 40 & Iprovalicarb & 93 & 92 & 1 & $\mathbf{0}$ & 87 & 13 \\
\hline 41 & Isoxaflutole & 93 & 83 & 10 & $\mathbf{0}$ & 82 & 14 \\
\hline 42 & Linuron & 102 & 97 & 4 & 1 & 85 & 12 \\
\hline 43 & Metamitron & 102 & 97 & 5 & $\mathbf{0}$ & 88 & 15 \\
\hline 44 & Methabenzthiazuron & 93 & 93 & $\mathbf{0}$ & $\mathbf{0}$ & 88 & 13 \\
\hline \multirow[t]{4}{*}{45} & Methamidophos & 102 & 90 & 12 & $\mathbf{0}$ & 75 & 12 \\
\hline & Methiocarb & 102 & 100 & 2 & 0 & 85 & 13 \\
\hline & Methiocarb-sulfone & 102 & 84 & 18 & 0 & 78 & 15 \\
\hline & Methiocarb-sulfoxide & 102 & 99 & 2 & 1 & 88 & 12 \\
\hline 46 & Methomyl & 102 & 89 & 0 & 13 & 101 & 14 \\
\hline 47 & Methoxyfenozide & 102 & 101 & 1 & $\mathbf{0}$ & 85 & 14 \\
\hline 48 & Metobromuron & 102 & 97 & 4 & 1 & 87 & 12 \\
\hline 49 & Metoxuron & 93 & 93 & $\mathbf{0}$ & $\mathbf{0}$ & 89 & 12 \\
\hline 50 & Monocrotophos & 102 & 101 & 1 & $\mathbf{0}$ & 90 & 12 \\
\hline \multirow[t]{4}{*}{51} & Monolinuron & 102 & 101 & 1 & $\mathbf{0}$ & 86 & 14 \\
\hline & Omethoate & 102 & 99 & 3 & 0 & 83 & 12 \\
\hline & Oxamyl & 102 & 100 & 2 & 0 & 89 & 12 \\
\hline & Oxamyl-oxime & 102 & 101 & 1 & 0 & 88 & 12 \\
\hline \multirow[t]{2}{*}{52} & Oxycarboxin & 102 & 102 & $\mathbf{0}$ & $\mathbf{0}$ & 91 & 12 \\
\hline & Oxydemeton-methyl & 102 & 97 & 5 & 0 & 86 & 13 \\
\hline 53 & Paclobutrazole & 102 & 101 & 1 & $\mathbf{0}$ & 87 & 12 \\
\hline \multirow[t]{6}{*}{54} & Pencycuron & 102 & 96 & 6 & $\mathbf{0}$ & 81 & 14 \\
\hline & Phenmedipham & 102 & 94 & 7 & 1 & 83 & 14 \\
\hline & Phenmedipham-metabolite & 102 & 100 & 2 & 0 & 93 & 15 \\
\hline & Phorate & 102 & 68 & 34 & 0 & 74 & 19 \\
\hline & Phorate-sulfone & 93 & 93 & 0 & 0 & 88 & 12 \\
\hline & Phorate-sulfoxide & 102 & 101 & 1 & 0 & 90 & 12 \\
\hline 55 & Phosphamidon & 93 & 93 & $\mathbf{0}$ & $\mathbf{0}$ & 89 & 10 \\
\hline \multirow[t]{3}{*}{56} & Picolinafen & 93 & 86 & 6 & 1 & 84 & 15 \\
\hline & Pirimicarb & 102 & 101 & 0 & 1 & 89 & 12 \\
\hline & Pirimicarb, desmethyl- & 102 & 100 & 1 & 1 & 90 & 12 \\
\hline 57 & Prochloraz & 101 & 94 & 7 & $\mathbf{0}$ & 83 & 14 \\
\hline 58 & Profoxydim & 99 & 54 & 32 & 13 & 99 & 21 \\
\hline 59 & Propamocarb & 101 & 9 & 92 & $\mathbf{0}$ & 70 & 15 \\
\hline 60 & Propoxur & 102 & 100 & 2 & $\mathbf{0}$ & 88 & 16 \\
\hline 61 & Pymetrozine & 102 & 73 & 29 & $\mathbf{0}$ & 89 & 20 \\
\hline 62 & Pyraclostrobin & 102 & 95 & 7 & $\mathbf{0}$ & 85 & 14 \\
\hline 63 & Pyridate-metabolite & 102 & 92 & 9 & 1 & 86 & 15 \\
\hline 64 & Rotenone & 102 & 93 & 9 & $\mathbf{0}$ & 81 & 15 \\
\hline 65 & Sethoxydim & 102 & 72 & 3 & 27 & 106 & 19 \\
\hline \multirow[t]{2}{*}{66} & Spinosyn-A & 93 & 88 & 5 & $\mathbf{0}$ & 82 & 17 \\
\hline & Spinosyn-D & 93 & 82 & 11 & $\mathbf{0}$ & 83 & 15 \\
\hline 67 & Tebuconazole & 93 & 90 & 3 & $\mathbf{0}$ & 86 & 16 \\
\hline 68 & Tebufenozide & 102 & 99 & 3 & $\mathbf{0}$ & 86 & 14 \\
\hline 69 & Temephos & 102 & 94 & 8 & $\mathbf{0}$ & 87 & 16 \\
\hline
\end{tabular}


Table 10 (continued)

\begin{tabular}{|c|c|c|c|c|c|c|c|}
\hline & & \# ACQ samples & \# Recov. $60-140 \%$ & \# Recov. $<60 \%$ & \# Recov. $>140 \%$ & Average recov. $(\%)^{\mathrm{a}}$ & $\operatorname{RSD}(\%)^{\mathrm{a}}$ \\
\hline \multirow[t]{4}{*}{70} & Tepraloxydim & 102 & 62 & $\mathbf{0}$ & 40 & 114 & 14 \\
\hline & Terbufos & 93 & 62 & 30 & 1 & 77 & 15 \\
\hline & Terbufos-sulfone & 93 & 90 & 3 & 0 & 86 & 13 \\
\hline & Terbufos-sulfoxide & 93 & 92 & 1 & 0 & 88 & 12 \\
\hline 71 & Thiabendazole & 98 & 92 & 5 & 1 & 86 & 13 \\
\hline 72 & Thiacloprid & 93 & 90 & 3 & $\mathbf{0}$ & 88 & 12 \\
\hline 73 & Thiametoxam & 93 & 91 & 2 & $\mathbf{0}$ & 89 & 13 \\
\hline \multirow[t]{5}{*}{74} & Thiocyclam & 93 & 64 & 29 & $\mathbf{0}$ & 78 & 16 \\
\hline & Thiodicarb & 102 & 62 & 40 & 0 & 82 & 16 \\
\hline & Thiofanox & 102 & 98 & 3 & 1 & 85 & 14 \\
\hline & Thiofanox-sulfone & 102 & 102 & 0 & 0 & 90 & 13 \\
\hline & Thiofanox-sulfoxide & 102 & 101 & 1 & 0 & 92 & 14 \\
\hline \multirow[t]{5}{*}{75} & Thiometon & 93 & 88 & 4 & 1 & 87 & 16 \\
\hline & Thiophanate-methyl & 102 & 83 & 19 & 0 & 77 & 12 \\
\hline & Tolylfluanid & 101 & 36 & 65 & 0 & 76 & 22 \\
\hline & Triadimefon & 102 & 99 & 3 & 0 & 85 & 13 \\
\hline & Triadimenol & 102 & 98 & 3 & 1 & 87 & 12 \\
\hline 76 & Triazoxide & 102 & 90 & 9 & 3 & 84 & 16 \\
\hline 77 & Trichlorfon & 102 & 101 & $\mathbf{0}$ & 1 & 87 & 12 \\
\hline 78 & Tricyclazole & 102 & 96 & 6 & $\mathbf{0}$ & 87 & 12 \\
\hline 79 & Triflumuron & 101 & 89 & 10 & 2 & 84 & 18 \\
\hline 80 & Triforine & 102 & 97 & 3 & 2 & 87 & 15 \\
\hline 81 & Vamidothion & 102 & 101 & 1 & $\mathbf{0}$ & 89 & 11 \\
\hline 82 & Sum aldicarb & 102 & 101 & 1 & $\mathbf{0}$ & 88 & 11 \\
\hline 83 & Sum butocarboxim & 102 & 101 & 1 & $\mathbf{0}$ & 90 & 11 \\
\hline 84 & Sum carbendazim & 101 & 97 & 4 & $\mathbf{0}$ & 83 & 12 \\
\hline 85 & Sum carbofuran & 102 & 102 & $\mathbf{0}$ & $\mathbf{0}$ & 92 & 10 \\
\hline 86 & Sum dimethoate & 102 & 100 & 2 & $\mathbf{0}$ & 86 & 10 \\
\hline 87 & Sum dichlofluanid & 102 & 89 & 1 & 12 & 107 & 17 \\
\hline 88 & Sum disulfoton & 93 & 89 & 4 & $\mathbf{0}$ & 86 & 13 \\
\hline 89 & Sum ethiofencarb & 102 & 102 & $\mathbf{0}$ & $\mathbf{0}$ & 89 & 11 \\
\hline 90 & Sum fenamiphos & 102 & 101 & 1 & $\mathbf{0}$ & 90 & 11 \\
\hline 91 & Sum fensulfothion & 102 & 102 & $\mathbf{0}$ & $\mathbf{0}$ & 86 & 11 \\
\hline 92 & Sum fenthion & 102 & 102 & $\mathbf{0}$ & $\mathbf{0}$ & 89 & 12 \\
\hline 93 & Sum methiocarb & 102 & 100 & 2 & $\mathbf{0}$ & 83 & 12 \\
\hline 94 & Sum methomyl & 102 & 100 & 2 & $\mathbf{0}$ & 87 & 12 \\
\hline 95 & Sum oxamyl & 102 & 101 & 1 & $\mathbf{0}$ & 88 & 10 \\
\hline 96 & Sum oxydemeton-methyl & 102 & 101 & 1 & $\mathbf{0}$ & 88 & 11 \\
\hline 97 & Sum phenmedipham & 102 & 101 & 1 & $\mathbf{0}$ & 88 & 13 \\
\hline 98 & Sum phorate & 102 & 97 & 5 & $\mathbf{0}$ & 81 & 12 \\
\hline 99 & Sum pirimicarb & 102 & 101 & 1 & $\mathbf{0}$ & 90 & 12 \\
\hline 100 & Sum terbufos & 93 & 88 & 5 & $\mathbf{0}$ & 81 & 13 \\
\hline 101 & Sum thiofanox & 102 & 102 & $\mathbf{0}$ & $\mathbf{0}$ & 89 & 11 \\
\hline 102 & Sum tolylfluanid & 101 & 95 & 6 & $\mathbf{0}$ & 80 & 15 \\
\hline 103 & Sum triadimefon & 102 & 99 & 3 & $\mathbf{0}$ & 86 & 13 \\
\hline
\end{tabular}

${ }^{a}$ Average and RSD for recoveries within $60-140 \%$ range Matrix-matched calibration, API3000

Level $=0.05 \mathrm{mg} \mathrm{kg}^{-1}$ for most pesticides/metabolites

Bold indicates pesticides, including metabolites that are part of residue definition, if appropriate

bined GCB/PSA dispersive clean-up enables prolonged injection of vegetable and fruit extracts $(10 \mathrm{mg}$ matrix equivalent) without maintenance. Retention time shifts induced by some matrices compared with the calibration standard are reduced by the clean-up procedure. Interferences are partially removed, resulting in cleaner (extracted ion) chromatograms. The last two benefits aid correct automatic peak assignment and confirmation. Addition of toluene during 
Table 11 Results from the analysis of Fapas (series 19) proficiency test samples (2003-2005)

\begin{tabular}{|c|c|c|c|c|c|c|}
\hline Sample & Pesticide & MRM & Spike level added $\left(\mu \mathrm{g} \mathrm{kg}^{-1}\right)$ & Inter-lab. result $\left(\mu \mathrm{g} \mathrm{kg}^{-1}\right)$ & TNO result $\left(\mu \mathrm{g} \mathrm{kg}^{-1}\right)$ & Z-score TNO \\
\hline \multirow[t]{5}{*}{ \#53 Apple } & Fenpropathrin & $\mathrm{GC}-\mathrm{MS}$ & 500 & 405 & 528 & 1.7 \\
\hline & Parathion-methyl & $\mathrm{GC}-\mathrm{MS}$ & 70 & 59 & 47 & -0.9 \\
\hline & Tetradifon & GC-MS & 140 & 115 & 91 & -0.9 \\
\hline & Triazofos & GC-MS & 140 & 119 & 74 & -1.7 \\
\hline & Vinchlozolin & GC-MS & 60 & 53 & 53 & 0.0 \\
\hline \multirow[t]{3}{*}{ \#52 Cucumber } & Iprodione & GC-MS & 100 & 94 & 89 & -0.3 \\
\hline & Methomyl & LC-MS-MS & 28 & 25 & 28 & 0.5 \\
\hline & Thiabendazole & LC-MS-MS & 50 & 128 & 113 & -0.5 \\
\hline \multirow[t]{3}{*}{ \#51 Pear } & Carbendazim & LC-MS-MS & 150 & 116 & 60 & -2.2 \\
\hline & Dodine & not in MRM & 60 & 59 & * & $*$ \\
\hline & Imazalil & LC-MS-MS & 400 & 237 & 273 & 0.8 \\
\hline \multirow[t]{4}{*}{ \#49 Melon } & Chlorpropham & GC-MS & 10 & 9 & 11 & 1.0 \\
\hline & Chlorpyrifos & $\mathrm{GC}-\mathrm{MS}$ & 8 & 8 & 7 & -0.7 \\
\hline & Dimethoate & LC-MS-MS & 15 & 19 & 15 & -0.9 \\
\hline & Pirimicarb & LC-MS-MS & 20 & 19 & 16 & -0.7 \\
\hline \multirow{5}{*}{ \#48 Tomato } & Azoxystrobin & GC-MS & Not given & 201 & 166 & -0.9 \\
\hline & Bifenthrin & $\mathrm{GC}-\mathrm{MS}$ & Not given & 83 & 99 & 0.9 \\
\hline & Buprofezin & GC-MS & Not given & 108 & 131 & 1 \\
\hline & Chlorpyrifos-methyl & GC-MS & Not given & 319 & 281 & -0.6 \\
\hline & Procymidone & GC-MS & Not given & 712 & 668 & -0.4 \\
\hline \multirow[t]{4}{*}{ \#47 Grapefruit } & Diazinon & $\mathrm{GC}-\mathrm{MS}$ & Not given & 262 & 294 & 0.6 \\
\hline & Heptenophos & GC-MS & Not given & 168 & 234 & 1.9 \\
\hline & Malathion & GC-MS & Not given & 715 & 690 & -0.2 \\
\hline & Methidathion & GC-MS & Not given & 567 & 540 & -0.3 \\
\hline \multirow[t]{5}{*}{ \#46 Lettuce } & Bromopropylate & GC-MS & 80 & 67 & 51 & -1.1 \\
\hline & Dimethoate & LC-MS-MS & 300 & 285 & 316 & 0.6 \\
\hline & Oxadixyl & GC-MS & 120 & 127 & 134 & 0.3 \\
\hline & Penconazole & GC-MS & 100 & 82 & 51 & -1.7 \\
\hline & Tolclofos-methyl & GC-MS & 160 & 137 & 75 & -2.1 \\
\hline \multirow[t]{6}{*}{ \#42 Apple } & Chlorfenvinphos & GC-MS & 90 & 71 & 50 & -1.3 \\
\hline & Chlorpyrifos & $\mathrm{GC}-\mathrm{MS}$ & 400 & 259 & 241 & -0.3 \\
\hline & Methamidophos & LC-MS-MS & 60 & 44 & 31 & -1.3 \\
\hline & Monocrotophos & LC-MS-MS & 80 & 58 & 56 & -0.1 \\
\hline & Omethoate & LC-MS-MS & 150 & 108 & 103 & -0.2 \\
\hline & Trifluralin & GC-MS & 100 & 59 & 62 & 0.2 \\
\hline \multirow[t]{4}{*}{ \#41 Basil } & Kresoxim-methyl & $\mathrm{GC}-\mathrm{MS}$ & 150 & 94 & 86 & -0.4 \\
\hline & Procymidone & GC-MS & 120 & 87 & 78 & -0.5 \\
\hline & Propyzamide & $\mathrm{GC}-\mathrm{MS}$ & 100 & 81 & 59 & -1.2 \\
\hline & Vinclozolin & $\mathrm{GC}-\mathrm{MS}$ & 60 & 47 & 44 & -0.3 \\
\hline \multirow[t]{4}{*}{ \#38 Tomato } & Azoxystrobin & $\mathrm{GC}-\mathrm{MS}$ & 150 & 137 & 132 & -0.2 \\
\hline & Bupirimate & GC-MS & 100 & 83 & 62 & -1.1 \\
\hline & Chlorpyrifos-methyl & GC-MS & 80 & 72 & 53 & -1.2 \\
\hline & Quinalphos & GC-MS & 140 & 124 & 105 & -0.7 \\
\hline \multirow[t]{4}{*}{ \#37 Lemon } & Diazinon & $\mathrm{GC}-\mathrm{MS}$ & 80 & 42 & 42 & 0.0 \\
\hline & Fenitrothion & GC-MS & 100 & 78 & 80 & 0.1 \\
\hline & Metalaxyl & GC-MS & 120 & 94 & 93 & 0 \\
\hline & Methidathion & $\mathrm{GC}-\mathrm{MS}$ & 150 & 109 & 154 & 1.9 \\
\hline \multirow[t]{3}{*}{ \#35 Lettuce } & Carbendazim & LC-MS-MS & 80 & 53 & 31 & -1.9 \\
\hline & lambda Cyhalothrin & GC-MS & 80 & 66 & 54 & -0.8 \\
\hline & Metalaxyl & GC-MS & 120 & 94 & 86 & -0.4 \\
\hline \multirow[t]{4}{*}{ \#34 Apple } & Diphenylamine & $\mathrm{GC}-\mathrm{MS}$ & 50 & 39 & 29 & -1.2 \\
\hline & Pirimiphos-methyl & $\mathrm{GC}-\mathrm{MS}$ & 50 & 41 & 42 & 0.1 \\
\hline & Propargite & GC-MS & 200 & 162 & 172 & 0.3 \\
\hline & Tetradifon & $\mathrm{GC}-\mathrm{MS}$ & 100 & 83 & 38 & -2.5 \\
\hline \multirow[t]{3}{*}{ \#29 Sweet pepper } & Dichloran & $\mathrm{GC}-\mathrm{MS}$ & 200 & 179 & 200 & 0.6 \\
\hline & Mecarbam & GC-MS & 100 & 90 & 120 & 1.5 \\
\hline & Methamidophos & LC-MS-MS & 60 & 51 & 54 & 0.3 \\
\hline
\end{tabular}


dispersive clean-up prevented unacceptable adsorption of planar pesticides by GCB yet removal of chlorophyll and other pigments was still sufficient. Use of liners with a sintered porous glass bed on the inner wall makes $20 \mu \mathrm{L}$ injection non-critical and robust. In GC, use of a universal matrix-matched standard (tomato) is a feasible means of compensating for the matrix effects of many other vegetable and fruit samples. For most pesticides, LOQs of $0.01 \mathrm{mg}$ $\mathrm{kg}^{-1}$ can be obtained by GC-MS with full-scan acquisition.

The same initial extract (i.e. without any clean-up) can be used for LC-MS-MS analysis, after changing the solvent to methanol-water. LC-MS-MS is relatively tolerant of injection of matrix-despite the absence of any clean-up no special maintenance was required. Matrix-induced suppression was observed for several matrices, however, especially herbs and citrus, and must be evaluated for all pesticidematrix combinations. In contrast with the $\mathrm{GC}-$ based method, use of a universal matrix-matched standard to compensate for matrix effects was not feasible.

Evaluation of analytical quality control data for 271 pesticides and degradation products in over one hundred matrices showed that, at the $0.05 \mathrm{mg} \mathrm{kg}^{-1}$ level, recovery was acceptable for 92\% (LC-MS-MS) and 93\% (GC-MS) of all pesticide-matrix combinations. It also revealed that the method fails in the other $7-8 \%$ because of lack of specificity (mostly in GC-MS) or because of poor extraction efficiency and/or degradation (LC-MS-MS). The only way to identify these limitations is by thorough and continual evaluation of the quantitative performance of the method for all the pesticides (rather then a "representative subset") in all the matrices.

Acknowledgements Jan Quirijns is acknowledged for development of the initial ethyl acetate-based method at the TNO laboratory and for investigation of sample homogenization. Gert Stil, Corina van Ballegooien, Piet van Prattenburg, Petra Dam, Rob van Dinter, Maarten Nooteboom, and Hans Kooiman are acknowledged for generation of the extensive set of analytical quality-control data during routine analysis of the samples.

\section{References}

1. Luke M, Froberg JE, Masumoto HT (1975) J Assoc Off Anal Chem 58:1020-1026

2. Specht W, Pelz S, Gilsbach W (1995) Fresenius J Anal Chem 353:183-190

3. Stan HJ (2000) J Chromatogr A 892:347-377

4. General Inspectorate for Health Protection (1996) analytical methods for pesticide residues in foodstuffs, 6th edn., Part 1, Ministry of Health, Welfare and Sport. The Hague, The Netherlands

5. Roos AH, van Munsteren AJ, Nabs FM, Tuinstra LGM Th (1987) Anal Chim Acta 196:95-102

6. Andersson A, Palsheden H, Fresenius (1991) J Anal Chem 339:365-367
7. Steinwandter H, Fresenius (1992) J Anal Chem 343:887-889

8. Hajslova J, Holadova K, Kocourek V, Poustka J, Godula M, Cuhra P, Kempny M (1998) J Chromatogr A 800:283-295

9. Pihlstrom T, Osterdahl BG (1999) J Agric Food Chem 47:2549-2552

10. Obana H, Akutsu K, Okihashi M, Hori S (2001) Analyst 126:1529-1534

11. Taylor MJ, Hunter K, Hunter KB, Lindsay D, le Bouhellec S (2002) J Chromatogr A 982:225-236

12. Zrostlikova J, Hajslova J, Cajka T (2003) J Chromatogr A 1019:173-186

13. Cajka T, Hajslova J (2004) J Chromatogr A 1058:251-261

14. Patel K, Fussell RJ, Goodall DM, Keely BJ (2003) Analyst 128:1228-1231

15. Mol HGJ, van Dam RCJ, Steijger OM (2003) J Chromatogr A 1015:119-127

16. Jansson Ch, Pihlstrom T, Osterdahl BG, Markides KE (2004) J Chromatogr A 1023:93-104

17. Agüera A, López S, Fernández-Alba AR, Contreras M, Crespo J, Piedra L (2004) J Chromatogr A 1045:125-135

18. Ferrer I, Garcia-Reyes JF, Mezcua M, Thurman EM, FernandezAlba AR (2005) J Chromatogr A 1082:81-90

19. Fernández Moreno JL, Arrebola Liébanas FJ, Garrido Frenich A, Martínez Vidal JL (2006) J Chromatogr A 1111:97-105

20. Zhang W-G, Chu X-G, Cai H-X, An J, Li Ch-J (2006) Rapid Commun Mass Spectrom 20:609-617

21. Sharif Z, Bin Che Man Y, Sheikh Abdul Hamid N, Cheow Keat Ch (2006) J Chromatogr A 1127:254-261

22. Berrada H, Fernandez M, Ruiz MJ, Molto JC, J Manes (2006) Food Add Contam 23:674-682

23. Martinez Vidal JL, Arrebola Liebanas FJ, Gonzalez Rodriguez MJ, Garrido Frenich A, Fernandez Moreno JL (2006) Rapid Commun Mass Spectrom 20:365-375

24. Cortes JM, Sanchez R, Diaz plaza EM, Villen J, Vazquez A (2006) J Agric Food Chem 54:1997-2002

25. Patel K, Fussell RJ, Goodall DM, Keely BJ (2006) J Sep Sci 29:90-95

26. Diez C, Traag WA, Zomer P, Marinero P, Atienza J (2006) J Chromatogr A 1131:11-23

27. Liao W, Joe T, Cusick WG (1991) J Assoc Off Anal Chem 74:554-565

28. Fillion J, Hindle R, Lacroix M, Selwyn J (1995) J Assoc Off Anal Chem 78:1252-1266

29. Anastassiades M, Lehotay SJ, Stajnbaher D, Schenk FJ (2003) J Assoc Off Anal Chem 86:412-431

30. Lehotay SJ, de Kok A, Hiemstra M, van Bodegraven P (2005) J Assoc Off Anal Chem 88:595-614

31. Hercegova A, Domotorova M, Kruzlicova D, Matisova E (2006) J Sep Sci 29:1102-1109

32. Reynolds 1997-2001: S.L. Reynolds, R. Fussell, M. Caldow, R. James, S. Nawaz, C. Ebeen, D. Pendlington, T. Stijve, S. Lovell, H. Diserens $(1997,1998,2000,2001)$ "Intercomparison study of two multi-residue methods for the enforcement of EU MRLs for pesticides in fruits, vegetables and grain", European Commission, Luxembourg

33. Pizzuti IR, de Kok A, Zanella R, Adaime MB, Hiemstra M, Wickert C, Prestes OD (2007) J Chromatogr A 1142:123-136

34. Annex I to Directive 90/642/EEC [Part of products to which maximum limits apply], as amended by Directive 93/58/EEC (OJ L 211, 23.8.1993, p. 6)

35. Commission directive 2002/63/EC of 11 July 2002 establishing Community methods of sampling for the official control of pesticide residues in and on products of plant and animal origin and repealing Directive 79/700/EEC, Official Journal of the European Communities L187, 16/07/2002, P. 30-43

36. SANCO/825/00 rev. 7, 17.03.2004, Guidance document on residue analytical methods 
37. SANCO/10232/2006, Quality control procedures for pesticide residue analysis 4 th edn

38. Okihashi M, Kitagawa Y, Akutsu K, Obana H, Tanaka Y (2005) J Pestic Sci 30:368-377

39. Fussell RJ, Addie KJ, Reynolds SL, Wilson MF (2002) J Agric Food Chem 50:441-448

40. Martinez Vidal JL, Arrebola FJ, Mateu-Sanchez M (2002) Rapid Commun Mass Spec 16:1106-1115

41. Lehotay SJ (2000) J Assoc Off Anal Chem 83:680-697

42. Kerkdijk H, Mol HGJ, van der Nagel B, submitted for publication

43. Barwick VJ, Ellison SLR, Lacey SJ, Mussell CR, Lucking CL (1999) J Sci Food Agric 79:1190-1196

44. Ueno E, Oshima H, Saito I, Matsumoto H (2004) J Assoc Off Anal Chem 87:1003-1015
45. Pang GF, Liu Y-M, Fan C-L, Zhang J-J, Cao Y-Z, Li X-M, Li Z-Y, Wu Y-P, Guo T-T (2006) Anal Bioanal Chem 384:13661408

46. Mol HGJ, Janssen H-G, Cramers CA, Brinkman UA Th (1995) J High Resolut Chromatogr 18:19-27

47. Teske J, Engewald W (2002) Trends Anal Chem 21:357

48. Godula M, Hajslova J, Mastouska K, Krivankova J (2001) J Sep Sci 24:355-366

49. Staniewski J, Rijks JA (1993) J High Resolut Chromatogr 16: 182-187

50. Poole CF (2007) J Chromatogr A DOI 10.1016/j.chroma.2007. 01.018

51. Niessen WMA, Manini P, Andreoli R (2006) Mass Spectrom Rev $25: 881-899$ 